\title{
DFT studies on thermal and oxidative degradation of monoethanolamine
}

\author{
Christopher. Parks ${ }^{a}{ }^{a}$, Ehsan. Alborzi ${ }^{a}$, Muhammad Akram ${ }^{a}$,
}

Mohammed. Pourkashanian, ${ }^{a}$

${ }^{a}$ Department of Mechanical Engineering, The University of Sheffield, Sheffield, S3 7RD,UK

E-mail: c.m.parks@sheffield.ac.uk, e.alborzi@sheffield.ac.uk, m.akram@sheffield.ac.uk

\section{Electronic Supplementary Information (ESI)}

Contents:

1. Raw DFT Data

2. Comparative data with M06 functional and Def2-TZVP basis set

3. Comparative data using SMD solvent model in place of PCM model.

All calculations were performed as described in the main text of the paper unless stated otherwise. 


\begin{tabular}{|c|c|}
\hline 1 & $\mathrm{O}_{2} \ldots \ldots . .$. \\
\hline 1.2 & 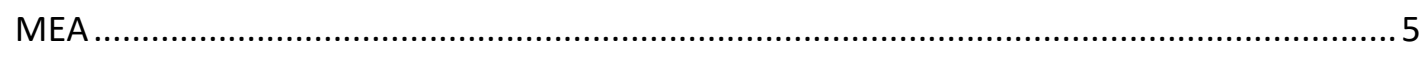 \\
\hline 1.3 & MEACO2 ...... \\
\hline 1.4 & 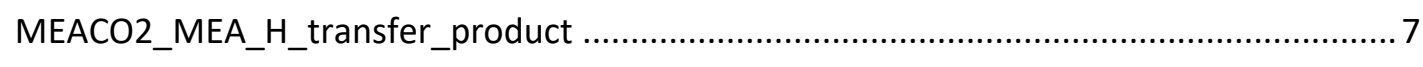 \\
\hline 1.5 & MEACO2_MEA_H_transfer_TS3. \\
\hline 1.6 & 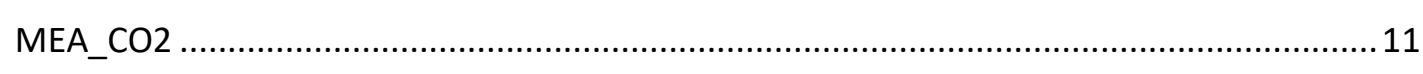 \\
\hline 1.7 & MEA_protonated............................... \\
\hline 1.8 & 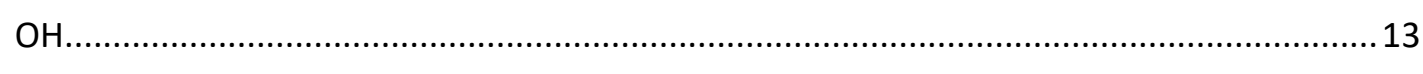 \\
\hline 1.9 & AEHEIA ........ \\
\hline 1.10 & AEHEIA_formation_TS....................... \\
\hline 1.11 & HEEDA ................... \\
\hline 1.12 & 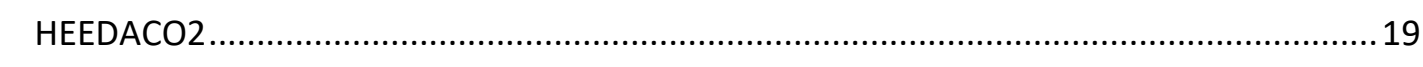 \\
\hline 1.13 & 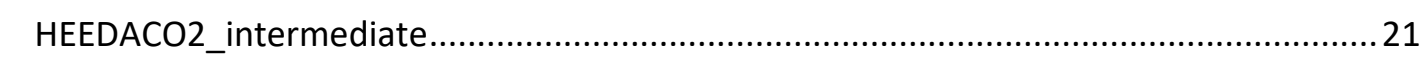 \\
\hline 1.14 & 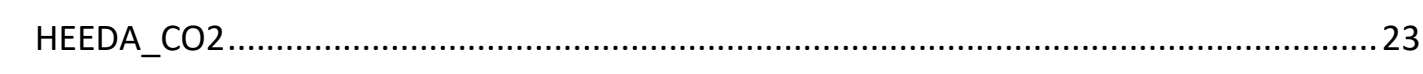 \\
\hline 1.15 & HEEDA_OXD_H_transfer_product ............. \\
\hline 1.16 & 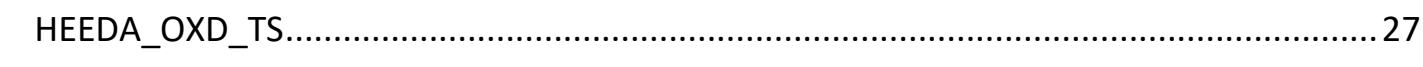 \\
\hline 1.17 & HEIA .......................... \\
\hline 1.18 & HEIA_Formation_TS .................................. \\
\hline 1.19 & MEA_CO2_Ring_formation_TS \\
\hline 1.20 & OZD \\
\hline 1.21 & 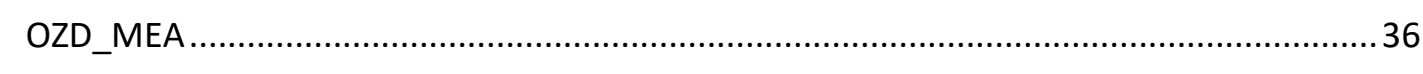 \\
\hline 1.22 & OZD_MEA_H2O ................ \\
\hline 1.23 & OZD_MEA_TS .................................. \\
\hline 1.24 & Pre_AEHEIA_1 …..................................... \\
\hline 1.25 & AEHEIA_1_3_H_transfer_TS ......... \\
\hline 1.26 & Pre_AEHEIA_2 \\
\hline 1.27 & Isocyanate ..................... \\
\hline 1.28 & Isocyanate_MEA.......... \\
\hline 1.29 & Isocyanate_MEA_BHEU_formation_TS .... \\
\hline 1.30 & Isocyanate_formation_TS...... \\
\hline 1.31 & Isocyanate_H2O ............................ \\
\hline 1.32 & Isocyanate_H2O_H2O ................. \\
\hline 1.33 & 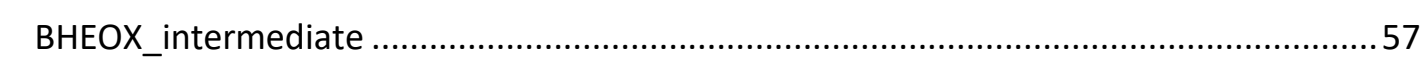 \\
\hline 1.34 & 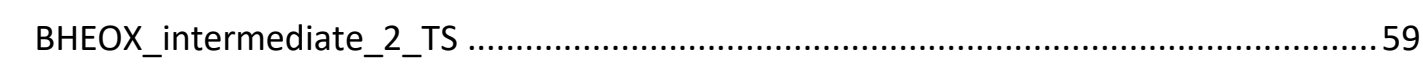 \\
\hline 1.35 & BHEOX_intermediate_3 ................ \\
\hline 36 & BHEOX_intermediate_4 …........................... \\
\hline
\end{tabular}




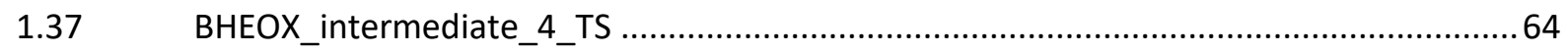

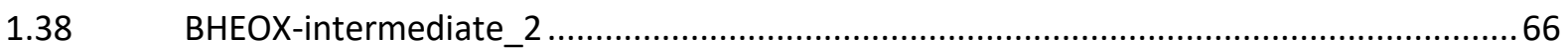

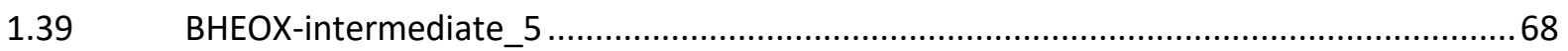

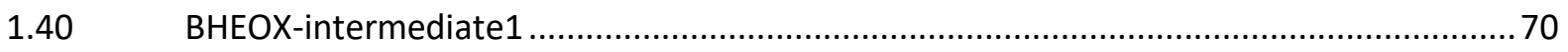

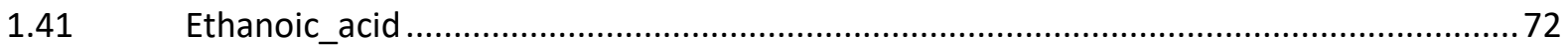

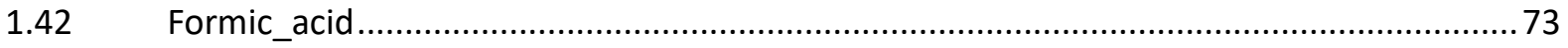

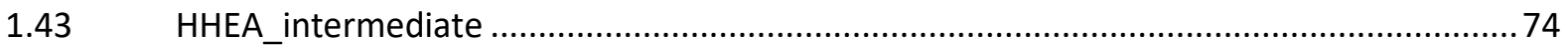

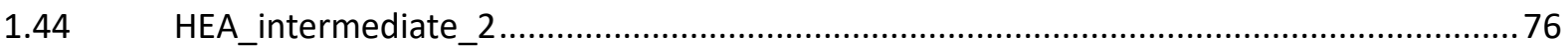

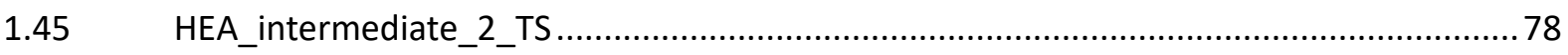

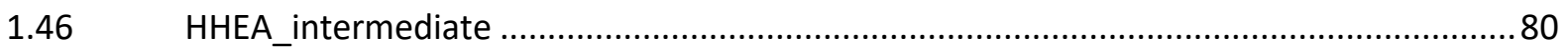

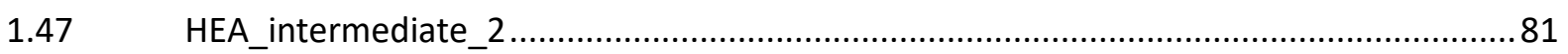

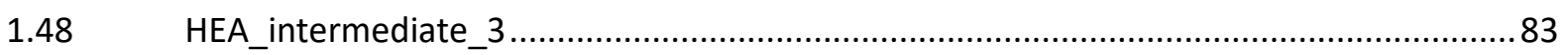

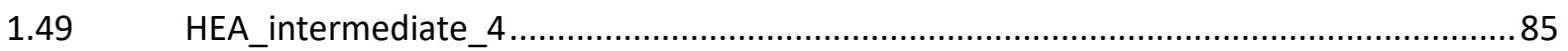

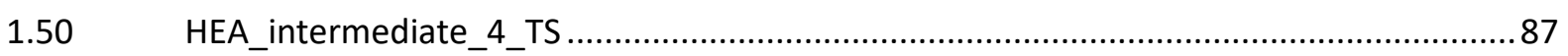

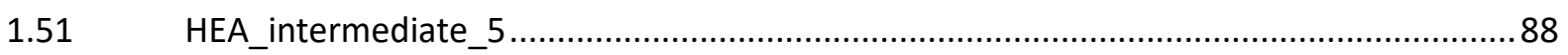

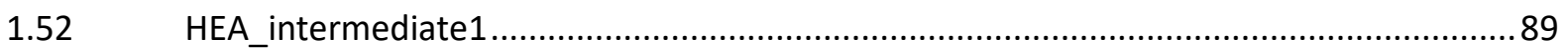

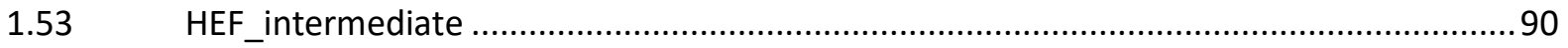

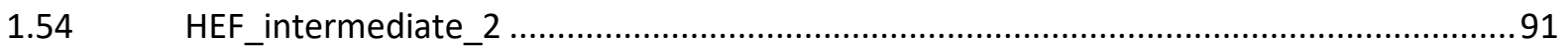

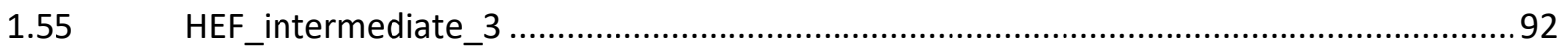

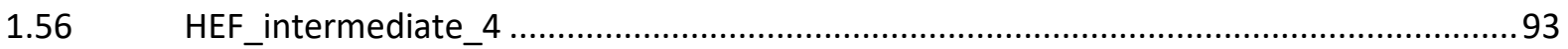

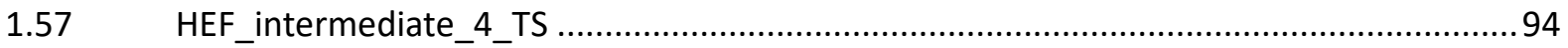

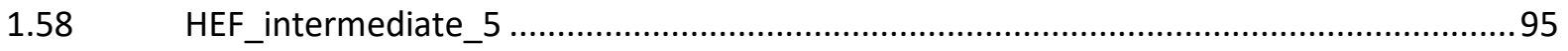

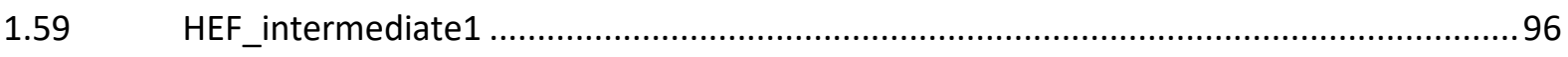

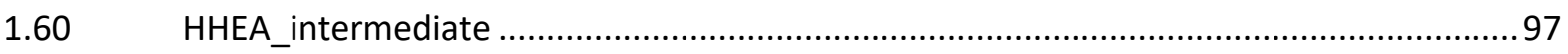

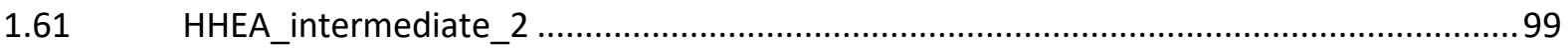

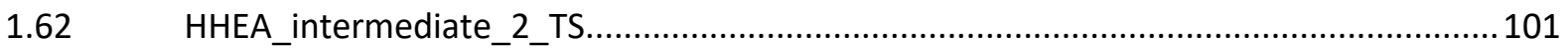

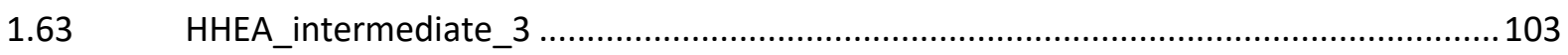

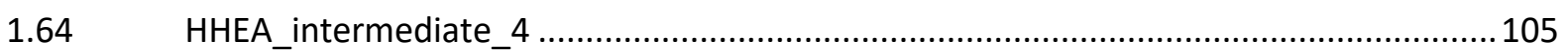

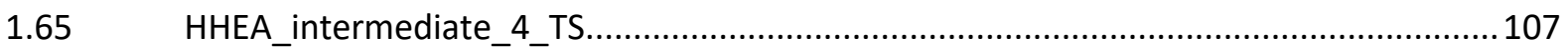

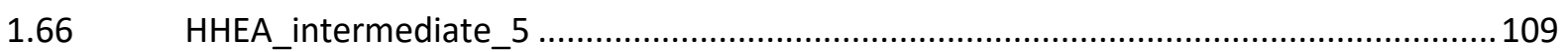

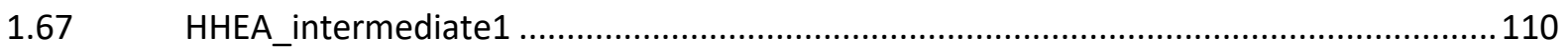

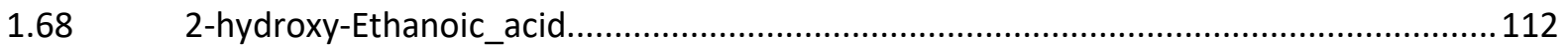

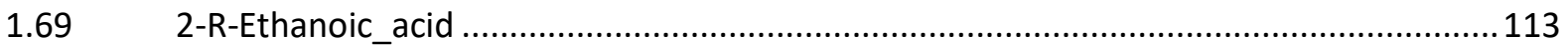


$1.1 \quad \mathrm{CO}_{2}$

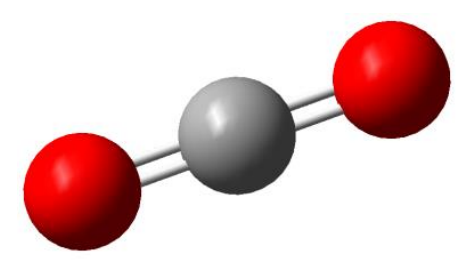

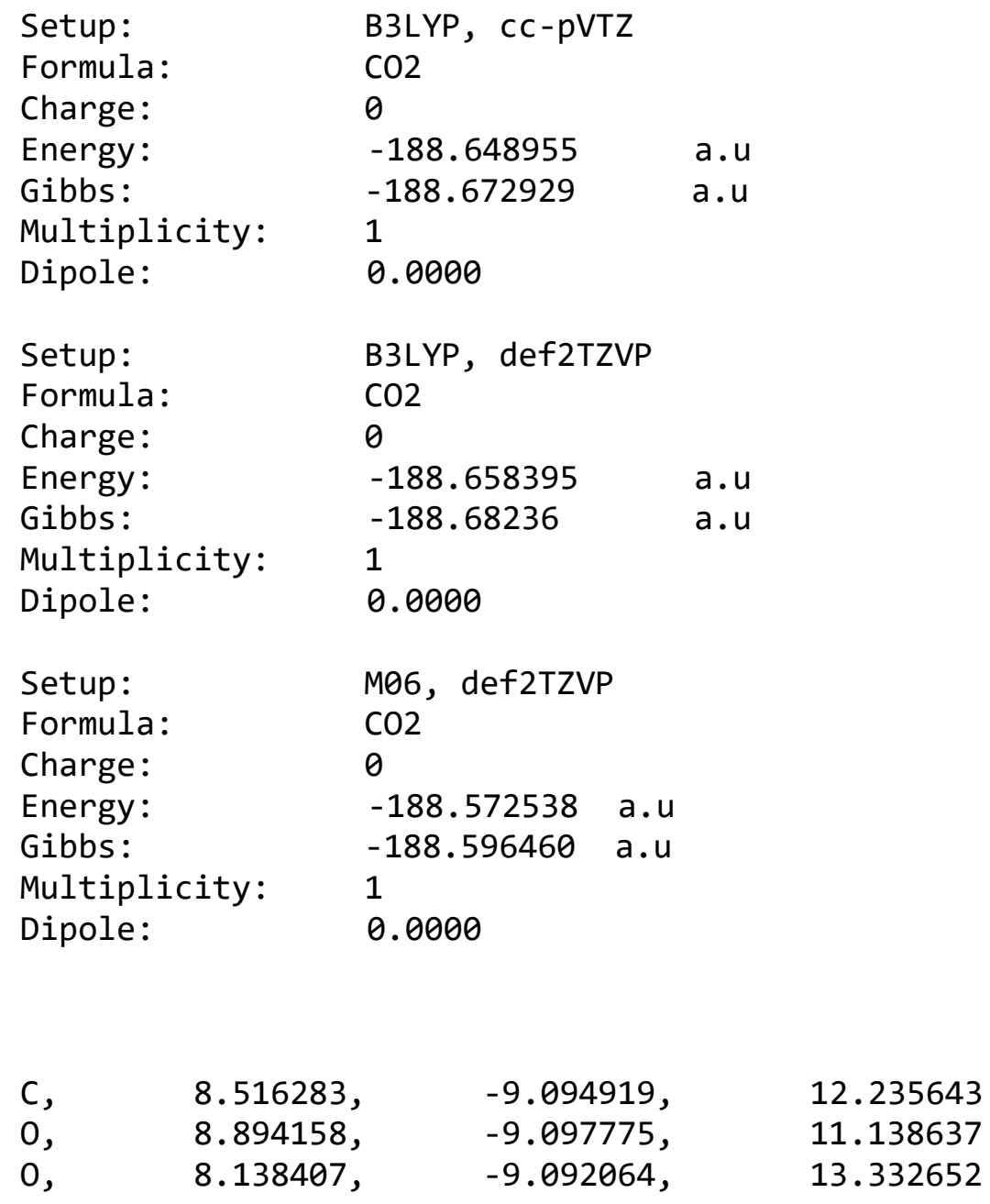




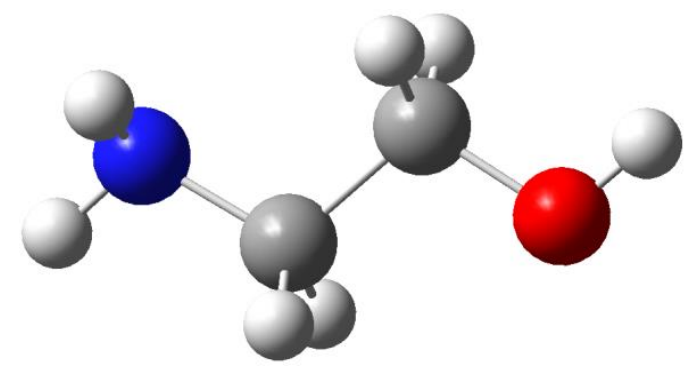

$\begin{array}{lll}\text { Formula: } & \text { C2H7NO } & \\ \text { Charge: } & 0 & \\ \text { Energy: } & -210.381663 & \text { a.u } \\ \text { Gibbs: } & -210.414974 & \text { a.u } \\ \text { Multiplicity: } & 1 & \\ \text { Dipole: } & 1.4511 & \\ & & \\ \text { Setup: } & \text { B3LYP, def2TZVP } & \\ \text { Formula: } & \text { C2H7NO } & \\ \text { Charge: } & 0 & \\ \text { Energy: } & -210.387652 \text { a.u } \\ \text { Gibbs: } & -210.420959 \text { a.u } \\ \text { Multiplicity: } & 1 & \\ \text { Dipole: } & 1.4890 & \\ & & \\ \text { Setup: } & \text { M06, def2TZVP } \\ \text { Formula: } & \text { C2H7NO } & \\ \text { Charge: } & 0 & \\ \text { Energy: } & -210.238802 & \text { a.u } \\ \text { Gibbs: } & -210.272082 \text { a.u } \\ \text { Multiplicity: } & 1 & \\ \text { Dipole: } & 1.4489 & \end{array}$

$\begin{array}{lllr}\text { C, } & 1.959249, & 1.737827, & -0.201285 \\ \text { H, } & 1.307446, & 2.043799, & 0.626299 \\ \text { H, } & 2.965761, & 1.589188, & 0.203248 \\ \text { C, } & 1.994251, & 2.831224, & -1.254383 \\ \text { H, } & 2.677115, & 2.527085, & -2.049857 \\ \text { H, } & 0.995373, & 2.915847, & -1.701165 \\ \text { O, } & 1.467581, & 0.548478, & -0.821826 \\ \text { H, } & 1.438756, & -0.149605, & -0.160959 \\ \text { H, } & 2.629464, & 4.770353, & -1.391844 \\ \text { H, } & 1.790471, & 4.467935, & -0.043255 \\ \text { N, } & 2.491519, & 4.079199, & -0.664548\end{array}$




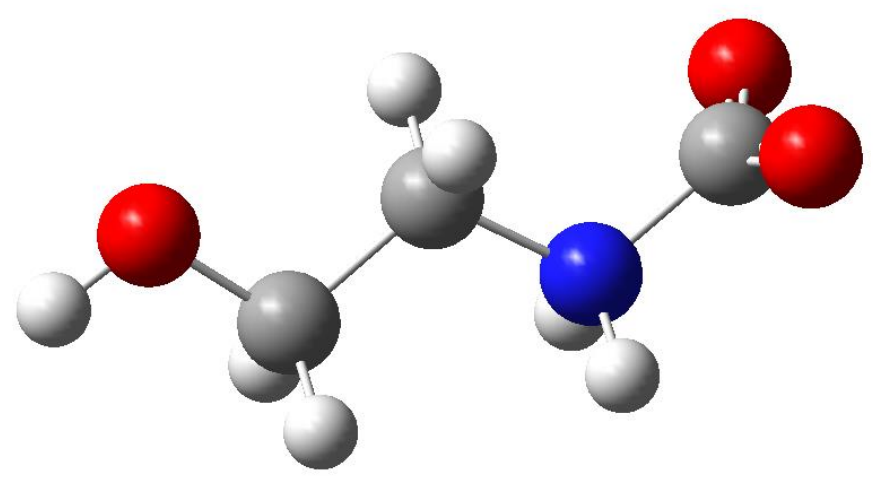

Formula:

Charge:

Energy:

Gibbs:

Multiplicity:

Dipole:

Setup:

Formula:

Charge:

Energy:

Gibbs:

Multiplicity:

Dipole:

\section{Setup:}

Formula:

Charge:

Energy:

Gibbs:

Multiplicity

Dipole :
C3H7NO3

0

$-399.034587 \quad$ a.u

$-399.082374 \quad$ a.u

1

3.7059

B3LYP, def2TZVP

C3H7NO3

0

-399.029133 a.u

-399.069635 a.u

1

11.2394

M06, def2TZVP

$\mathrm{C} 3 \mathrm{H} 7 \mathrm{NO} 3$

0

-398.788083 a.u -398.828061 a.u

1

11.0668

$\begin{array}{llll}\text { C, } & 10.144442, & -5.063696, & 11.701820 \\ \text { H, } & 11.137201, & -5.133379, & 12.162377 \\ \text { H, } & 9.504950, & -4.477224, & 12.372378 \\ \text { C, } & 9.561427, & -6.460622, & 11.535121 \\ \text { H, } & 8.580954, & -6.382552, & 11.061650 \\ \text { H, } & 10.195281, & -7.031542, & 10.854039 \\ \text { O, } & 10.220311, & -4.460951, & 10.408197 \\ \text { H, } & 10.588241, & -3.577271, & 10.503222 \\ \text { C, } & 8.325462, & -9.735440, & 12.162550 \\ \text { O, } & 8.290930, & -9.460999, & 11.034862 \\ \text { O, } & 8.326730, & -10.086019, & 13.269554 \\ \text { H, } & 8.820770, & -6.715722, & 13.431686 \\ \text { H, } & 10.318036, & -7.317621, & 13.239149 \\ \text { N, } & 9.419637, & -7.212068, & 12.781606\end{array}$




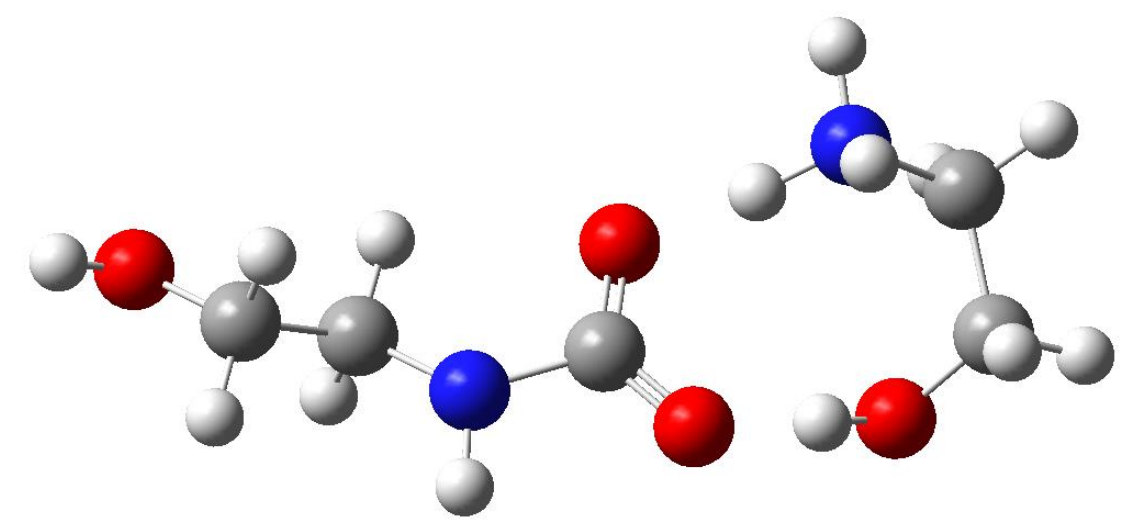

\section{Formula:}

Charge:

Energy:

Gibbs:

Multiplicity:

Dipole:

Setup:

Formula:

Charge:

Energy:

Gibbs:

Multiplicity:

Dipole:

Setup:

Formula:

Charge:

Energy:

Gibbs:

Multiplicity:

Dipole:
C5H14N2O4

0

$-609.438551 \quad$ a.u

-609.494921 a.u

1

8.9290

B3LYP, def2TZVP

C5H14N2O4

0

-609.463613 a.u

-609.520051 a.u

1

9.9221

M06, def2TZVP

C5H14N2O4

0

-609.068343 a.u

$-609.125326 \quad$ a.u

1

10.1952

$\begin{array}{lrrr}\text { C, } & -1.012817, & 0.839954, & -0.062520 \\ \text { H, } & -0.775630, & 1.496801, & 0.781178 \\ \text { H, } & -1.435531, & -0.086936, & 0.342398 \\ \text { C, } & 0.261027, & 0.534433, & -0.835333 \\ \text { H, } & 0.005258, & -0.066327, & -1.716189 \\ \text { H, } & 0.699147, & 1.464555, & -1.190206 \\ \text { O, } & -1.926236, & 1.463232, & -0.967198 \\ \text { H, } & -2.747020, & 1.640026, & -0.498141 \\ \text { C, } & 2.676305, & 0.108511, & -0.147683 \\ \text { O, } & 3.027393, & 1.159376, & -0.755142 \\ \text { O, } & 3.435038, & -0.742163, & 0.375644 \\ \text { H, } & 1.044317, & -1.087133, & 0.241794 \\ \text { N, } & 1.233888, & -0.123117, & 0.015663\end{array}$




$\begin{array}{lllr}\text { C, } & 6.697579, & -0.185120, & -0.437999 \\ \text { H, } & 6.729918, & -0.354451, & 0.646199 \\ \text { H, } & 7.655421, & -0.518121, & -0.842071 \\ \text { C, } & 6.590302, & 1.307766, & -0.712691 \\ \text { H, } & 7.484497, & 1.823288, & -0.366025 \\ \text { H, } & 6.464718, & 1.487362, & -1.777998 \\ \text { O, } & 5.677665, & -0.937964, & -1.053207 \\ \text { H, } & 4.856298, & -0.876813, & -0.504751 \\ \text { H, } & 5.396901, & 2.921896, & -0.141904 \\ \text { H, } & 4.442640, & 1.533887, & -0.382019 \\ \text { H, } & 5.447635, & 1.733404, & 0.980465 \\ \text { N, } & 5.407877, & 1.911549, & -0.021520\end{array}$




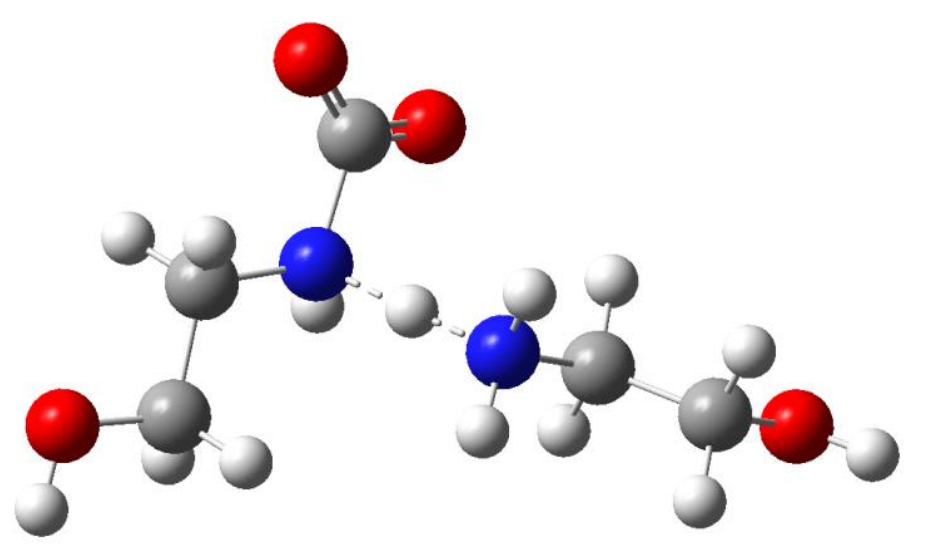

$\begin{array}{lll}\text { Formula: } & \text { C5H14N2O4 } & \\ \text { Charge: } & 0 & \\ \text { Energy: } & -609.393415 & \text { a.u } \\ \text { Gibbs: } & -609.450711 & \text { a.u } \\ \text { Multiplicity: } & 1 & \\ \text { Dipole: } & 8.5475 & \\ & & \\ \text { Setup: } & \text { B3LYP, def2TZVP } \\ \text { Formula: } & \text { C5H14N2O4 } & \\ \text { Charge: } & 0 & \\ \text { Energy: } & -609.438882 & \text { a.u } \\ \text { Gibbs: } & -609.497637 & \text { a.u } \\ \text { Multiplicity: } & 1 & \\ \text { Dipole: } & 14.2952 & \\ & & \\ \text { Setup: } & \text { M06, def2TZVP } \\ \text { Formula: } & \text { C5H14N2O4 } & \\ \text { Charge: } & 0 & \\ \text { Energy: } & -398.752276 & \text { a.u } \\ \text { Gibbs: } & -398.792886 & \text { a.u } \\ \text { Multiplicity: } & 1 & \\ \text { Dipole: } & 5.9231 & \end{array}$

$\begin{array}{lrrr}\text { C, } & -1.591711, & 1.133363, & -0.466689 \\ \text { H, } & -1.243394, & 0.947465, & -1.492453 \\ \text { H, } & -1.974694, & 2.153383, & -0.410736 \\ \text { C, } & -0.426569, & 0.989576, & 0.500054 \\ \text { H, } & -0.753980, & 1.275008, & 1.497040 \\ \text { H, } & -0.111660, & -0.056106, & 0.526518 \\ \text { O, } & -2.575751, & 0.172770, & -0.085604 \\ \text { H, } & -3.411368, & 0.410274, & -0.495824 \\ \text { C, } & 0.598080, & 3.342766, & 0.354654 \\ \text { O, } & -0.432200, & 3.726677, & 0.895534 \\ \text { O, } & 1.636582, & 3.926600, & -0.016219 \\ \text { H, } & 1.009344, & 1.682276, & -0.816629 \\ \text { H, } & 1.962922, & 1.671367, & 0.860804\end{array}$




$\begin{array}{lllr}\text { N, } & 0.724975, & 1.826682, & 0.147801 \\ \text { C, } & 5.500242, & 1.650937, & 0.923370 \\ \text { H, } & 5.664545, & 1.591877, & 2.006900 \\ \text { H, } & 5.593312, & 2.701438, & 0.624793 \\ \text { C, } & 4.106185, & 1.149535, & 0.586976 \\ \text { H, } & 3.931076, & 1.241812, & -0.483674 \\ \text { H, } & 4.012047, & 0.098684, & 0.856162 \\ \text { O, } & 6.405925, & 0.820346, & 0.214298 \\ \text { H, } & 7.300186, & 1.150749, & 0.338656 \\ \text { H, } & 3.064409, & 2.909225, & 1.027176 \\ \text { H, } & 3.104652, & 1.821895, & 2.291979 \\ \text { N, } & 3.051795, & 1.913858, & 1.282390\end{array}$




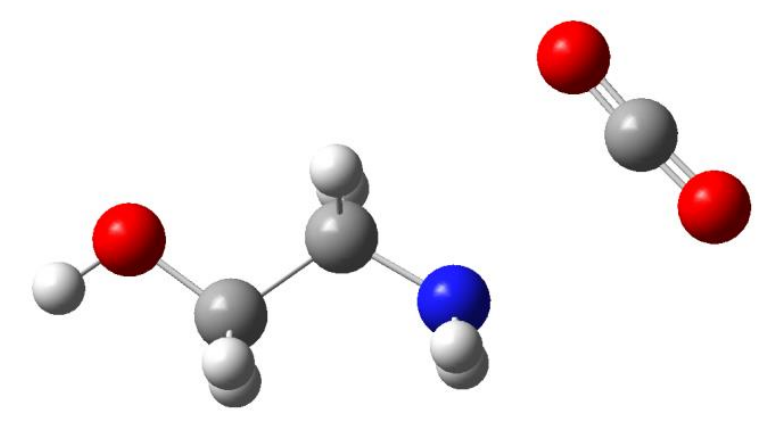

$\begin{array}{lll}\text { Formula: } & \text { C3H7NO3 } & \\ \text { Charge: } & 0 & \\ \text { Energy: } & -399.034587 & \text { a.u } \\ \text { Gibbs: } & -399.082374 & \text { a.u } \\ \text { Multiplicity: } & 1 & \\ \text { Dipole: } & 3.7059 & \end{array}$

$\begin{array}{lll}\text { Setup: } & \text { B3LYP, def2TZVP } \\ \text { Formula: } & \text { C3H7NO3 } & \\ \text { Charge: } & 0 & \\ \text { Energy: } & -399.048889 \text { a.u } \\ \text { Gibbs: } & -399.096968 \text { a.u } \\ \text { Multiplicity: } & 1 & \\ \text { Dipole: } & 3.7935 & \\ & & \\ \text { Setup: } & \text { M06, def2TZVP } \\ \text { Formula: } & \text { C3H7NO3 } & \\ \text { Charge: } & 0 & \\ \text { Energy: } & -398.812962 \text { a.u } \\ \text { Gibbs: } & -398.859505 \text { a.u } \\ \text { Multiplicity: } & 1 & \\ \text { Dipole: } & 3.6982 & \end{array}$

$\begin{array}{llll}\text { C, } & 10.144442, & -5.063696, & 11.701820 \\ \text { H, } & 11.137201, & -5.133379, & 12.162377 \\ \text { H, } & 9.504950, & -4.477224, & 12.372379 \\ \text { C, } & 9.561427, & -6.460622, & 11.535121 \\ \text { H, } & 8.580954, & -6.382552, & 11.061650 \\ \text { H, } & 10.195281, & -7.031542, & 10.854039 \\ \text { O, } & 10.220311, & -4.460951, & 10.408197 \\ \text { H, } & 10.588241, & -3.577271, & 10.503222 \\ \text { C, } & 8.325462, & -9.735440, & 12.162550 \\ \text { O, } & 8.290930, & -9.460999, & 11.034862 \\ \text { O, } & 8.326730, & -10.086019, & 13.269554 \\ \text { H, } & 8.820770, & -6.715722, & 13.431686 \\ \text { H, } & 10.318036, & -7.317621, & 13.239149 \\ \text { N, } & 9.419637, & -7.212068, & 12.781606\end{array}$




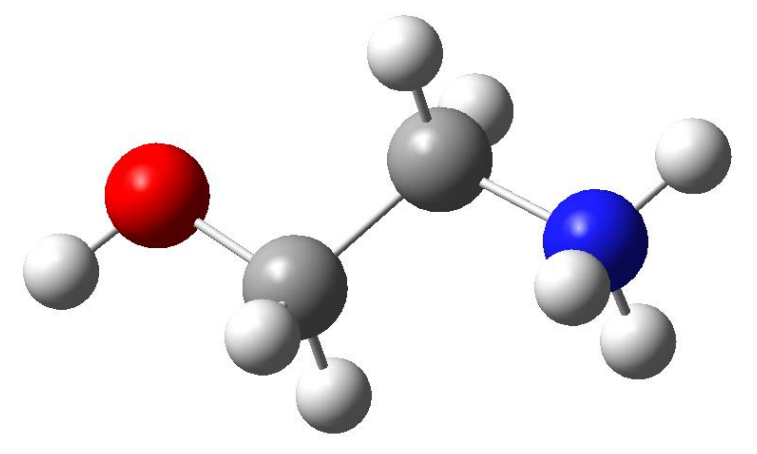

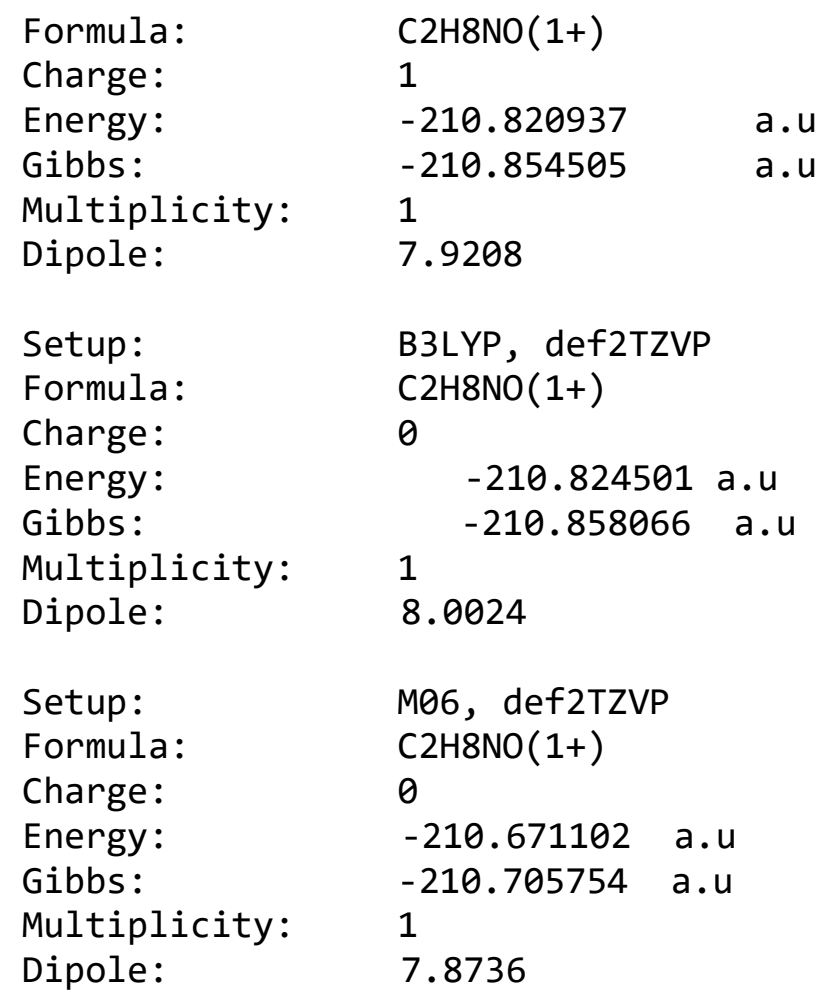

$\begin{array}{lrrr}\text { C, } & 1.952758, & 1.725063, & -0.179795 \\ \text { H, } & 1.272791, & 2.044599, & 0.617057 \\ \text { H, } & 2.945908, & 1.576495, & 0.257482 \\ \text { C, } & 2.016681, & 2.785972, & -1.264449 \\ \text { H, } & 2.704607, & 2.494771, & -2.052775 \\ \text { H, } & 1.034514, & 2.965357, & -1.691785 \\ \text { O, } & 1.485728, & 0.554305, & -0.828676 \\ \text { H, } & 1.423116, & -0.153123, & -0.179197 \\ \text { H, } & 3.441762, & 4.004482, & -0.304997 \\ \text { H, } & 2.549614, & 4.806263, & -1.429503 \\ \text { H, } & 1.889281, & 4.441443, & 0.031328 \\ \text { N, } & 2.505656, & 4.091043, & -0.703362\end{array}$



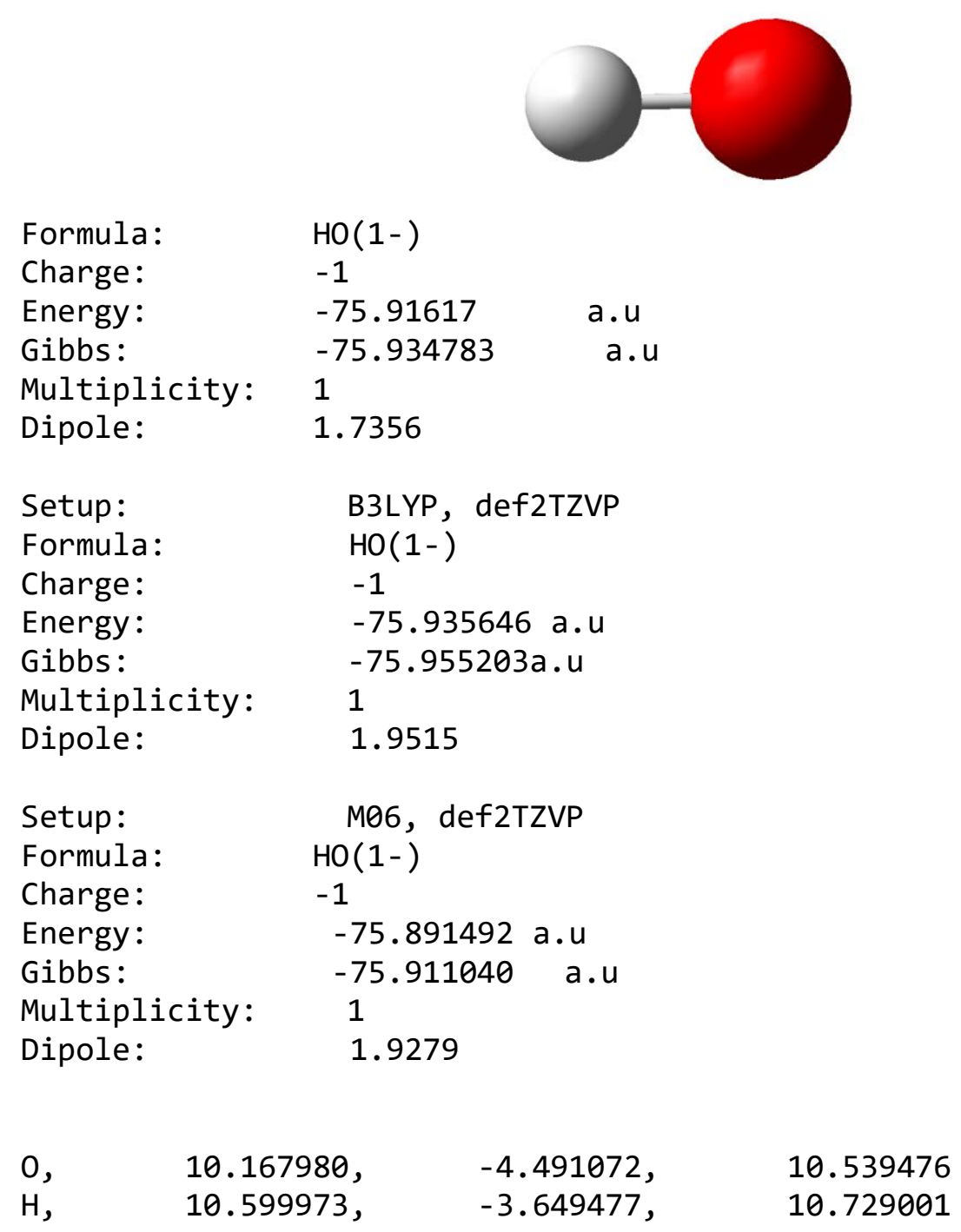


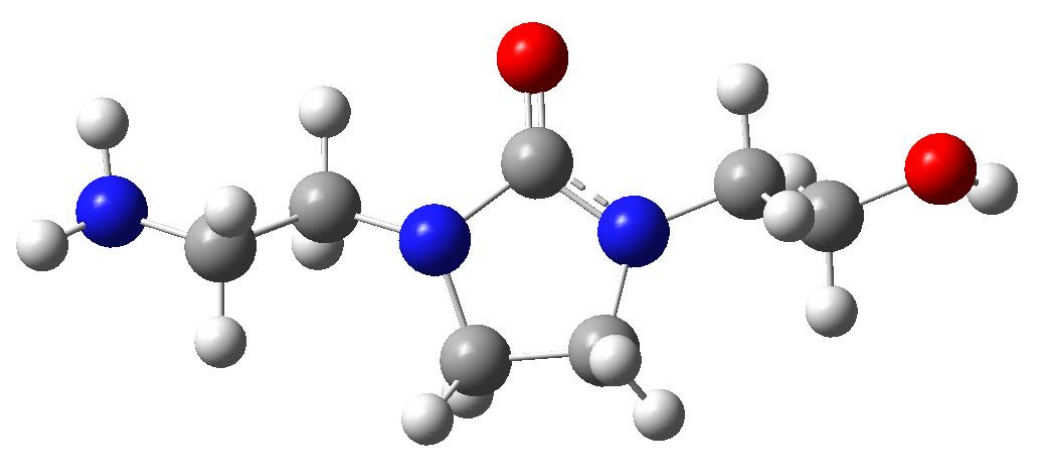

Formula:

Charge:

Energy:

Gibbs:

Multiplicity:

Dipole:

Setup:

Formula:

Charge:

Energy:

Gibbs:

Multiplicity:

Dipole:

Setup:

Formula:

Charge:

Energy:

Gibbs:

Multiplicity:

Dipole:
C7H15N3O2

0

-590.494215 a.u

$-590.548784 \quad$ a.u

1

5.6047

B3LYP, def2TZVP

C7H15N302

0

-590.515421 a.u

-590.570570 a.u

1

5.9675

M06, def2TZVP

C7H15N3O2

0

-590.103727 a.u

-590.159323 a.u

1

5.6432

$\begin{array}{llrr}\text { C, } & -3.343175, & 0.699169, & -0.029438 \\ \text { C, } & -5.507119, & 0.731327, & 1.143365 \\ \text { H, } & -5.590563, & 1.016548, & 2.195578 \\ \text { H, } & -5.346813, & -0.344461, & 1.095605 \\ \text { N, } & -4.359717, & 1.386001, & 0.551979 \\ \text { C, } & -6.798714, & 1.086361, & 0.419002 \\ \text { H, } & -6.736210, & 0.746431, & -0.619785 \\ \text { H, } & -6.938416, & 2.172399, & 0.412025 \\ \text { O, } & -7.858017, & 0.436924, & 1.118186 \\ \text { H, } & -8.688837, & 0.651634, & 0.683649 \\ \text { C, } & 0.123435, & 1.910529, & -0.909525 \\ \text { H, } & 0.425221, & 1.452026, & 0.040737 \\ \text { H, } & 0.094991, & 2.990000, & -0.748087\end{array}$




$\begin{array}{lllr}\text { O, } & -3.314278, & -0.478263, & -0.340759 \\ \text { C, } & -3.968561, & 2.745790, & 0.906240 \\ \text { H, } & -3.683280, & 2.792961, & 1.962006 \\ \text { H, } & -4.778020, & 3.451306, & 0.733362 \\ \text { C, } & -1.275100, & 1.411061, & -1.262784 \\ \text { H, } & -1.602310, & 1.887226, & -2.196940 \\ \text { H, } & -1.247879, & 0.334345, & -1.433766 \\ \text { N, } & 1.042275, & 1.643393, & -2.021224 \\ \text { H, } & 1.943753, & 2.064957, & -1.832082 \\ \text { H, } & 1.211920, & 0.645800, & -2.092415 \\ \text { C, } & -2.770716, & 2.992968, & -0.013121 \\ \text { H, } & -2.047116, & 3.671383, & 0.434085 \\ \text { H, } & -3.095555, & 3.415372, & -0.972866 \\ \text { N, } & -2.223195, & 1.654167, & -0.193796\end{array}$




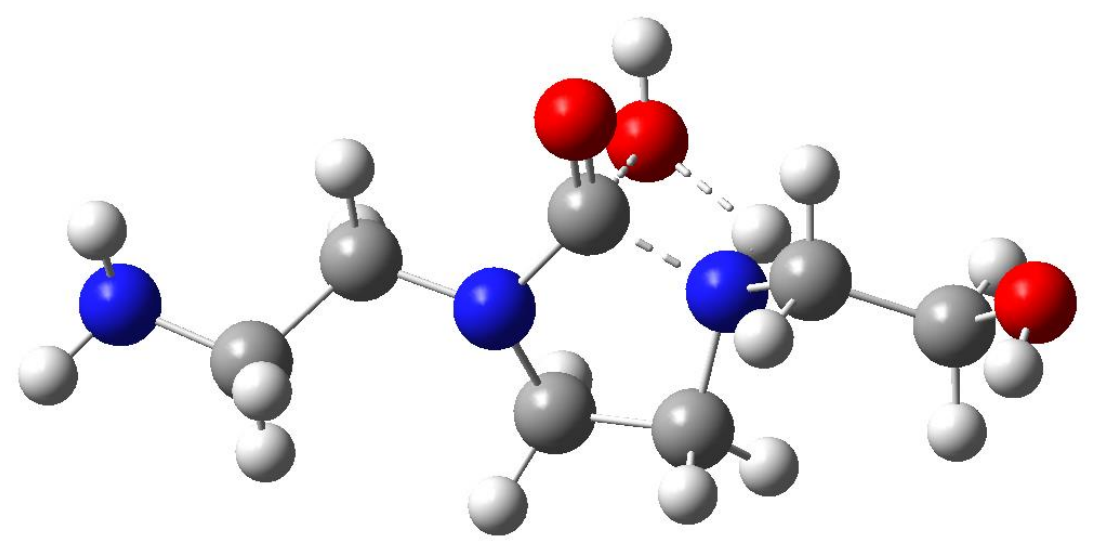

Formula:

Charge:

Energy:

Gibbs:

Multiplicity:

Dipole:

Setup:

Formula:

Charge:

Energy:

Gibbs:

Multiplicity:

Dipole:

Setup:

Formula:

Charge:

Energy:

Gibbs:

Multiplicity:

Dipole:
C7H17N303

0

$-666.877646$

$-666.934686$

1

9.2808

B3LYP, def2TZVP

C7H17N303

0

a.u

a.u

1

M06, def2TZVP

C7H17N303

0

-666.438842 a.u -666.496739 a.u

1

9.4748

$\begin{array}{lllr}\text { C, } & -0.511017, & -1.007435, & 0.256719 \\ \text { C, } & -2.884264, & -1.655103, & -0.480973 \\ \text { H, } & -2.595067, & -1.960963, & -1.486082 \\ \text { H, } & -3.023200, & -0.576834, & -0.475451 \\ \text { C, } & -4.169071, & -2.351777, & -0.043361 \\ \text { H, } & -4.422866, & -2.046833, & 0.972651 \\ \text { H, } & -4.044226, & -3.437988, & -0.044737 \\ \text { O, } & -5.255614, & -1.956184, & -0.869325 \\ \text { H, } & -5.168979, & -2.391743, & -1.723835 \\ \text { C, } & 2.927229, & -2.057462, & -0.557297 \\ \text { H, } & 2.580540, & -2.094863, & -1.597643 \\ \text { H, } & 3.010555, & -3.088633, & -0.210055 \\ \text { O, } & -0.666509, & -0.826515, & 2.264006 \\ \text { C, } & -1.222886, & -3.340420, & 0.421655\end{array}$




$\begin{array}{lrrr}\text { H, } & -1.350599, & -3.741774, & -0.580567 \\ \text { H, } & -1.751037, & -3.963814, & 1.135834 \\ \text { C, } & 1.888495, & -1.312707, & 0.275086 \\ \text { H, } & 2.180624, & -1.314799, & 1.330734 \\ \text { H, } & 1.837489, & -0.274175, & -0.050879 \\ \text { N, } & 4.235798, & -1.418116, & -0.385354 \\ \text { H, } & 4.951411, & -1.970188, & -0.842563 \\ \text { H, } & 4.240355, & -0.516554, & -0.850198 \\ \text { C, } & 0.254369, & -3.151521, & 0.751096 \\ \text { H, } & 0.855265, & -3.949902, & 0.324606 \\ \text { H, } & 0.422187, & -3.103783, & 1.830425 \\ \text { N, } & 0.553804, & -1.872714, & 0.125081 \\ \text { O, } & -0.640099, & 0.099447, & -0.222872 \\ \text { N, } & -1.764600, & -1.953010, & 0.434865 \\ \text { H, } & -1.934111, & -1.642570, & 1.419368 \\ \text { H, } & -0.736427, & 0.131742, & 2.303779\end{array}$




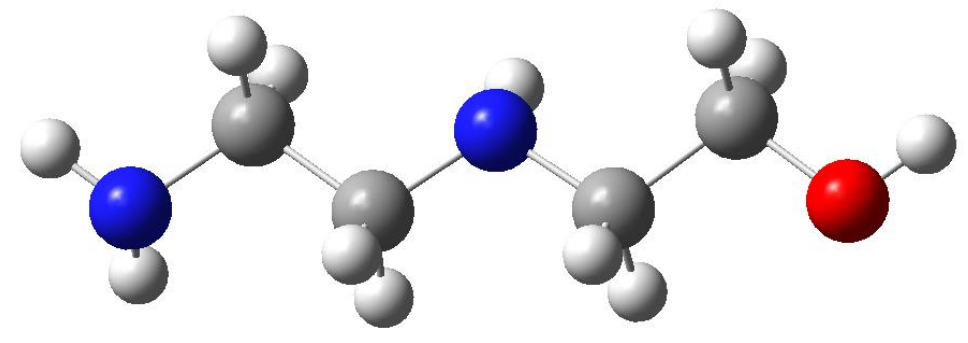

Formula:

Charge:

Energy:

Gibbs:

Multiplicity:

Dipole :

Setup:

Formula:

Charge:

Energy:

Gibbs:

Multiplicity:

Dipole:

Setup:

Formula:

Charge:

Energy:

Gibbs:

Multiplicity

Dipole :
$\mathrm{C} 4 \mathrm{H} 12 \mathrm{~N} 2 \mathrm{O}$

0

-344.329012 a.u

-344.372749 a.u

1

3.2567

B3LYP, def2TZVP

$\mathrm{C} 4 \mathrm{H} 12 \mathrm{~N} 2 \mathrm{O}$

0

-344.336446 a.u

-344.381080 a.u

1

3.3937

M06, def2TZVP

$\mathrm{C} 4 \mathrm{H} 12 \mathrm{~N} 2 \mathrm{O}$

0

-344.081110 a.u

-344.125719 a.u

1

3.3217

$\begin{array}{ll}\text { C, } & -3.996264, \\ \text { H, } & -4.266983, \\ \text { H, } & -2.905849, \\ \text { C, } & -4.436072, \\ \text { H, } & -4.222515, \\ \text { H, } & -5.519557, \\ \text { O, } & -4.644623, \\ \text { H, } & -4.380069, \\ \text { N, } & -3.804452, \\ \text { H, } & -2.797780, \\ \text { C, } & -4.228458, \\ \text { H, } & -5.308041, \\ \text { H, } & -4.041003, \\ \text { C, } & -3.532041, \\ \text { H, } & -2.444251, \\ \text { H, } & -3.794064, \\ \text { N, } & -3.977221, \\ \text { H, } & -3.567070, \\ \text { H, } & -3.626485,\end{array}$

C, -3.996264,

1.674543,

1.889705,

1.565153,

2.825423,

2.567386,

2.939804,

0.492097 ,

-0.243431 ,

4.070610,

3.972156,

5.226041,

5.347419,

5.091226,

6.495210,

6.338833 ,

6.673344 ,

7.647215,

8.496738,

7.562960,
$-0.063449$

0.975159

$-0.110197$

$-0.950535$

$-1.999410$

$-0.867078$

$-0.534894$

0.025470

$-0.529176$

$-0.609976$

$-1.314577$

$-1.198479$

$-2.393249$

$-0.841844$

$-0.875535$

0.202563

$-1.636255$

$-1.266344$

$-2.584443$ 


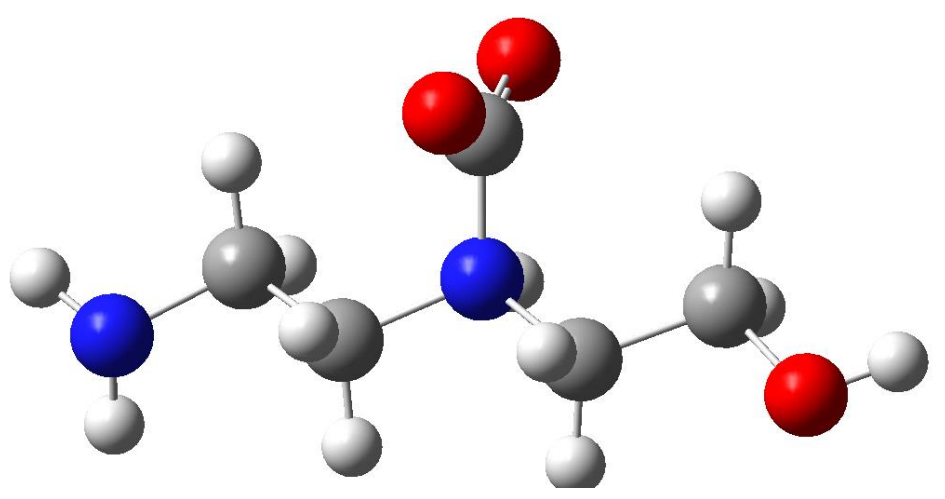

Formula:

Charge:

Energy:

Gibbs:

Multiplicity

Dipole:

Setup:

Formula:

Charge:

Energy:

Gibbs:

Multiplicity:

Dipole:

Setup:

Formula:

Charge:

Energy:

Gibbs:

Multiplicity:

Dipole:
$\mathrm{C} 5 \mathrm{H} 12 \mathrm{~N} 2 \mathrm{O} 3$

0

$-532.965593$

$-533.016575$

a.u

a.u

1

8.7997

B3LYP, def2TZVP

C5H12N2O3

0

-532.983456 a.u

-533.034264 a.u

1

8.9726

M06, def2TZVP

C5H12N2O3

0

-532.636106 a.u -532.686125 a.u

1

8.6363

$\begin{array}{lllr}\text { C, } & -3.848726, & 1.596583, & -0.017462 \\ \text { H, } & -3.964433, & 1.715494, & 1.063152 \\ \text { H, } & -2.782451, & 1.470904, & -0.234728 \\ \text { C, } & -4.392030, & 2.814685, & -0.746516 \\ \text { H, } & -4.301968, & 2.677879, & -1.821401 \\ \text { H, } & -5.431116, & 2.989564, & -0.484768 \\ \text { O, } & -4.611228, & 0.499626, & -0.503036 \\ \text { H, } & -4.346169, & -0.292069, & -0.024674 \\ \text { N, } & -3.636552, & 4.060850, & -0.380165 \\ \text { H, } & -2.649731, & 3.908893, & -0.591652 \\ \text { C, } & -4.109194, & 5.237848, & -1.196547 \\ \text { H, } & -5.146151, & 5.407908, & -0.924727 \\ \text { H, } & -4.050649, & 4.942300, & -2.242248\end{array}$




$\begin{array}{lllr}\text { C, } & -3.264501, & 6.478341, & -0.934673 \\ \text { H, } & -2.204201, & 6.239502, & -1.086321 \\ \text { H, } & -3.380314, & 6.784774, & 0.104929 \\ \text { N, } & -3.760080, & 7.556146, & -1.790847 \\ \text { H, } & -3.365116, & 8.439499, & -1.493334 \\ \text { H, } & -3.451205, & 7.414683, & -2.745974 \\ \text { C, } & -3.699058, & 4.336543, & 1.062397 \\ \text { O, } & -2.587792, & 4.298576, & 1.600604 \\ \text { O, } & -4.837305, & 4.554204, & 1.480328\end{array}$



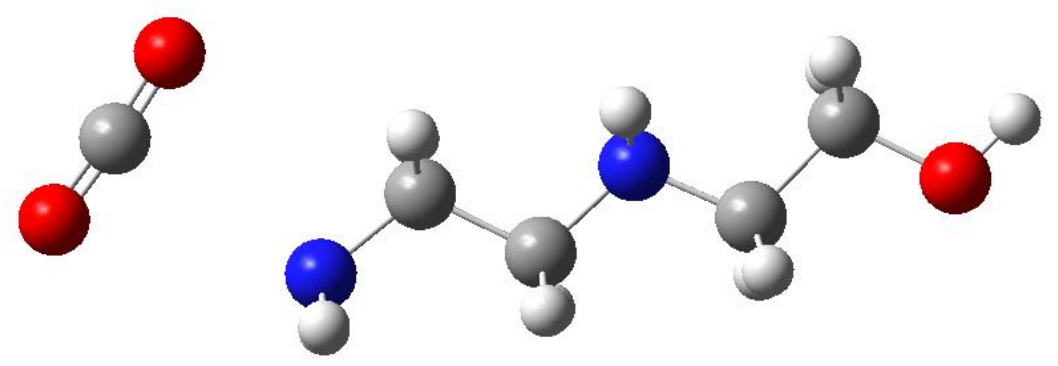

Formula:

Charge:

$\mathrm{C} 5 \mathrm{H} 12 \mathrm{~N} 2 \mathrm{O} 3$

Energy:

0

Gibbs:

$-532.981792$

a.u

$-533.040187$

a.u

Multiplicity:

1

Dipole:

1.8928

\section{Setup:}

Formula:

Charge:

Energy:

Gibbs:

B3LYP, def2TZVP

C5H12N2O3

0

-532.998482 a.u

-533.057311 a.u

Multiplicity: 1

Dipole:

1.8997

\section{Setup:}

Formula:

M06, def2TZVP

Charge:

C5H12N2O3

Energy:

0

Gibbs:

-532.656221 a.u

Multiplicity: $\quad 1$

Dipole:

-532.713629 a.u

1

1.9086

$\begin{array}{ll}\text { C, } & -1.470345, \\ \text { H, } & -1.527186, \\ \text { H, } & -1.663308, \\ \text { C, } & -0.058648, \\ \text { H, } & -0.005310, \\ \text { H, } & 0.149020, \\ \text { C, } & 3.456487, \\ \text { O, } & 2.895911, \\ \text { H, } & 0.849731, \\ \text { N, } & 1.005372, \\ \text { O, } & 4.091831, \\ \text { C, } & -4.800849, \\ \text { H, } & -4.736594, \\ \text { H, } & -4.530292, \\ \text { C, } & -3.829455, \\ \text { H, } & -3.886592,\end{array}$

0.789751 ,

$-1.032476$

-0.285084 ,

$-1.228069$

1.295217,

$-1.993277$

1.137773,

$-0.561830$

2.204374,

$-0.328335$

0.606096 ,

0.368353

1.556281,

$-0.326901$

2.002587,

0.586786

1.325513,

$-2.391268$

0.832035 ,

$-1.519374$

1.132807,

$-1.201775$

1.122670,

0.711594

2.187365,

0.967208

0.547437 ,

1.602805

0.803907 ,

$-0.410761$

-0.264519 ,

$-0.632560$ 


$\begin{array}{lrrr}\text { H, } & -4.149327, & 1.336069, & -1.319920 \\ \text { N, } & -2.462365, & 1.122081, & -0.013984 \\ \text { H, } & -2.401087, & 2.114366, & 0.189564 \\ \text { H, } & 1.003442, & -0.156199, & -1.746043 \\ \text { O, } & -6.113420, & 0.789294, & 0.256880 \\ \text { H, } & -6.737839, & 0.985716, & 0.961559\end{array}$




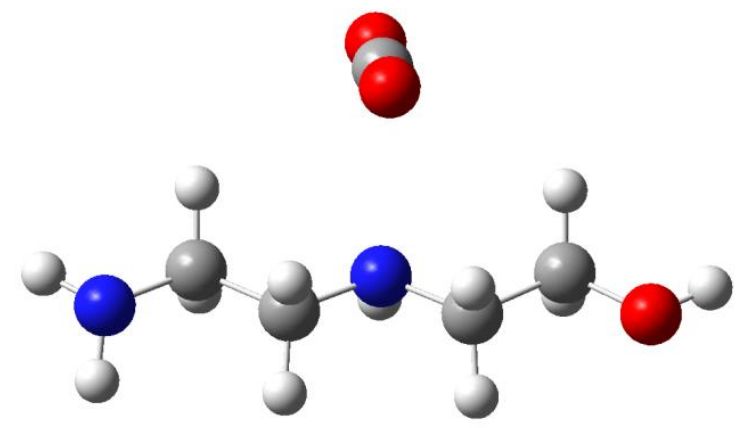

\begin{tabular}{|c|c|}
\hline Formula: & $\mathrm{C} 5 \mathrm{H} 12 \mathrm{~N} 2 \mathrm{O} 3$ \\
\hline Charge: & 0 \\
\hline Energy: & -532.982813 \\
\hline Gibbs: & -533.039824 \\
\hline Multiplicity: & 1 \\
\hline Dipole: & 3.4509 \\
\hline Setup: & B3LYP, def2TZVP \\
\hline Formula: & $\mathrm{C} 5 \mathrm{H} 12 \mathrm{~N} 2 \mathrm{O} 3$ \\
\hline Charge: & 0 \\
\hline Energy: & -532.999707 a.u \\
\hline Gibbs: & -533.056700 a.u \\
\hline Multiplicity: & 1 \\
\hline Dipole: & 3.5546 \\
\hline Setup: & M06, def2TZVP \\
\hline Formula: & $\mathrm{C} 5 \mathrm{H} 12 \mathrm{~N} 2 \mathrm{O} 3$ \\
\hline Charge: & 0 \\
\hline Energy: & -532.658692 a.u \\
\hline Gibbs: & -532.712736 a.u \\
\hline Multiplicity: & 1 \\
\hline Dipole: & 3.5084 \\
\hline
\end{tabular}

$\begin{array}{lllr}\text { C, } & -3.766207, & 1.593905, & -0.294292 \\ \text { H, } & -3.584328, & 1.744026, & 0.774773 \\ \text { H, } & -2.795899, & 1.429514, & -0.778383 \\ \text { C, } & -4.432487, & 2.826049, & -0.879824 \\ \text { H, } & -4.686930, & 2.626461, & -1.931457 \\ \text { H, } & -5.371129, & 3.004849, & -0.350879 \\ \text { O, } & -4.638785, & 0.485682, & -0.515964 \\ \text { H, } & -4.236100, & -0.299559, & -0.133428 \\ \text { N, } & -3.583059, & 3.999241, & -0.715042 \\ \text { H, } & -2.698232, & 3.843493, & -1.185624 \\ \text { C, } & -4.188246, & 5.227253, & -1.221345 \\ \text { H, } & -5.110667, & 5.409454, & -0.665900\end{array}$




$\begin{array}{lllr}\text { H, } & -4.467468, & 5.145685, & -2.284475 \\ \text { C, } & -3.253913, & 6.416074, & -1.038778 \\ \text { H, } & -2.288370, & 6.188181, & -1.512524 \\ \text { H, } & -3.056758, & 6.555705, & 0.025591 \\ \text { N, } & -3.881558, & 7.638189, & -1.555567 \\ \text { H, } & -3.289042, & 8.436421, & -1.359715 \\ \text { H, } & -3.948466, & 7.586080, & -2.566439 \\ \text { C, } & -3.512950, & 4.420911, & 2.066915 \\ \text { O, } & -2.355591, & 4.405025, & 2.158922 \\ \text { O, } & -4.673786, & 4.450093, & 2.055773\end{array}$




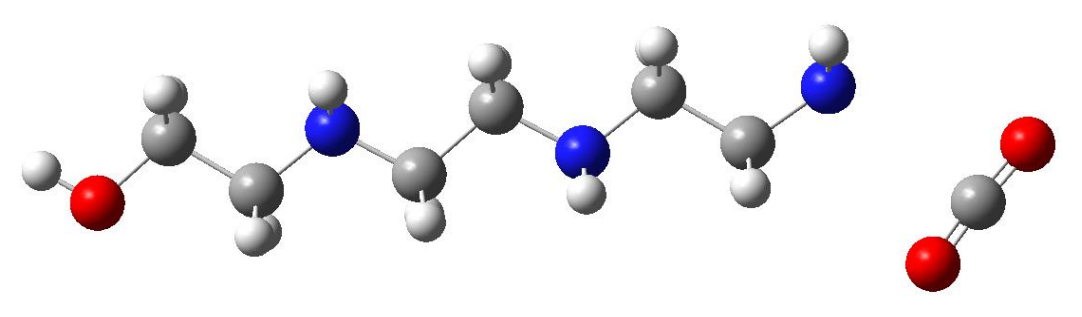

\begin{tabular}{|c|c|c|c|}
\hline Formula: & $\mathrm{C} 7 \mathrm{H} 17 \mathrm{~N} 303$ & & \\
\hline Charge: & 0 & & \\
\hline Energy: & -666.930304 & & a.u \\
\hline Gibbs: & -666.995807 & & a.u \\
\hline Multiplicity: & 1 & & \\
\hline Dipole: & 4.2617 & & \\
\hline Setup: & B3LYP, def2TZ & ZVP & \\
\hline Formula: & $\mathrm{C} 7 \mathrm{H} 17 \mathrm{~N} 303$ & & \\
\hline Charge: & 0 & & \\
\hline Energy: & -666.948486 & a.u & \\
\hline Gibbs: & -667.017768 & a.u & \\
\hline Multiplicity: & 1 & & \\
\hline Dipole: & 4.3256 & & \\
\hline Setup: & M06, def2TZVP & & \\
\hline Formula: & $\mathrm{C} 7 \mathrm{H} 17 \mathrm{~N} 303$ & & \\
\hline Charge: & 0 & & \\
\hline Energy: & -666.499613 & a.u & \\
\hline Gibbs: & -666.566481 & a.u & \\
\hline Multiplicity: & 1 & & \\
\hline Dipole: & 4.2577 & & \\
\hline
\end{tabular}

$\begin{array}{lllr}\text { C, } & -3.338623, & 0.918750, & -1.085330 \\ \text { H, } & -2.978036, & 0.083165, & -0.478040 \\ \text { H, } & -3.460120, & 0.532121, & -2.111103 \\ \text { C, } & -2.282365, & 2.023158, & -1.091805 \\ \text { H, } & -2.649733, & 2.875363, & -1.669513 \\ \text { H, } & -2.134470, & 2.381020, & -0.071350 \\ \text { C, } & 0.727579, & 3.868566, & -1.444260 \\ \text { O, } & 1.683009, & 3.247974, & -1.668829 \\ \text { H, } & -1.085017, & 1.309345, & -2.596261 \\ \text { N, } & -0.982650, & 1.633238, & -1.640964 \\ \text { O, } & -0.176600, & 4.559088, & -1.212115 \\ \text { C, } & -6.914166, & 0.921917, & 0.147085 \\ \text { H, } & -7.235910, & 1.816119, & -0.412615 \\ \text { H, } & -6.701330, & 1.251404, & 1.166514 \\ \text { C, } & -5.637043, & 0.372520, & -0.473422 \\ \text { H, } & -5.280382, & -0.465686, & 0.129588 \\ \text { H, } & -5.862883, & -0.033302, & -1.473976 \\ \text { N, } & -4.594037, & 1.392598, & -0.510969\end{array}$




$\begin{array}{llrr}\text { H, } & -4.926067, & 2.181900, & -1.055632 \\ \text { H, } & -0.595787, & 0.856933, & -1.115937 \\ \text { N, } & -7.952610, & -0.102845, & 0.198683 \\ \text { H, } & -8.137206, & -0.429445, & -0.744102 \\ \text { C, } & -9.199780, & 0.371907, & 0.787379 \\ \text { H, } & -9.642648, & 1.215829, & 0.236202 \\ \text { H, } & -8.992617, & 0.728273, & 1.799324 \\ \text { C, } & -10.217236, & -0.752160, & 0.863968 \\ \text { H, } & -10.415726, & -1.142953, & -0.141464 \\ \text { H, } & -9.812042, & -1.570621, & 1.467429 \\ \text { O, } & -11.408682, & -0.219306, & 1.444601 \\ \text { H, } & -12.058513, & -0.925686, & 1.506225\end{array}$




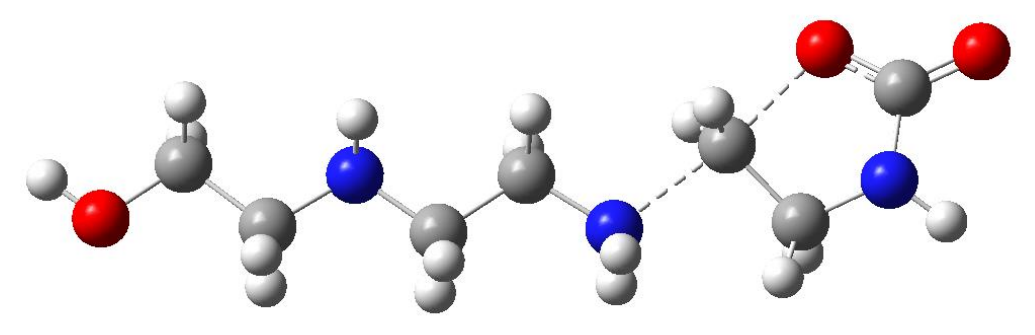

Formula:

Charge:

Energy:

Gibbs:

Multiplicity:

Dipole:

Setup:

Formula:

Charge:

Energy:

Gibbs:

Multiplicity:

Dipole:

Setup:

Formula:

Charge:

Energy:

Gibbs:

Multiplicity:

Dipole:
C7H17N303

0

$-666.881865$

$-666.942685$

a.u

a.u

1

15.2934

B3LYP, def2TZVP

C7H17N303

0

-666.963467 a.u

-666.902372 a.u

1

15.4061

M06, def2TZVP

C7H17N303

0

-666.442253 a.u

-666.503172 a.u

1

15.0875

C, -2.628563,

0.696444 ,

$-0.116929$

$\mathrm{H}, \quad-3.008288$,

0.052617 ,

0.651049

$\mathrm{H}, \quad-2.732791$,

0.387583 ,

$-1.141325$

C, -1.845544,

1.950106,

0.213218

$\mathrm{H}, \quad-2.288709$,

2.817833,

$-0.272531$

H, $\quad-1.875977$,

2.117852 ,

1.295234

C, $\quad-0.011616$,

0.466567 ,

$-0.183325$

O, -0.958696,

$-0.392900$

$-0.018530$

$\mathrm{H}, \quad 0.167732$,

2.508831,

$-0.126350$

$\mathrm{N}, \quad-0.495946$,

1.768302,

$-0.285930$

0 1.197764,

0.222845 ,

$-0.281315$

1.093005 ,

$-0.392697$

1.756316,

$-1.270485$

1.710879,

0.483162

0.524453 ,

$-0.272655$

-0.092124 ,

0.622860

$\mathrm{H}, \quad-5.442128$,

-0.113972 ,

$-1.129105$ 


$\begin{array}{llrr}\text { N, } & -4.469138, & 1.555782, & -0.203333 \\ \text { H, } & -4.489833, & 2.157420, & -1.020445 \\ \text { H, } & -4.591089, & 2.146535, & 0.613147 \\ \text { N, } & -7.900704, & 0.011547, & -0.439360 \\ \text { H, } & -7.718784, & -0.561796, & -1.256570 \\ \text { C, } & -9.281558, & 0.482684, & -0.487629 \\ \text { H, } & -9.493034, & 1.105188, & -1.369906 \\ \text { H, } & -9.467092, & 1.103109, & 0.392152 \\ \text { C, } & -10.244946, & -0.690580, & -0.470904 \\ \text { H, } & -10.053033, & -1.341498, & -1.332496 \\ \text { H, } & -10.085429, & -1.280622, & 0.437178 \\ \text { O, } & -11.570130, & -0.160798, & -0.520721 \\ \text { H, } & -12.191360, & -0.894968, & -0.508350\end{array}$




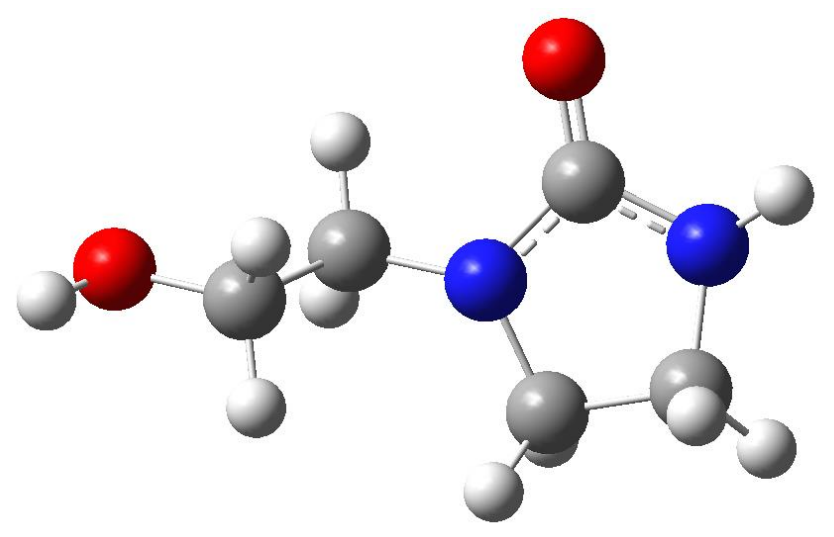

\begin{tabular}{|c|c|c|c|}
\hline Formula: & \multicolumn{3}{|l|}{ C5H9NO3 } \\
\hline Charge: & \multicolumn{3}{|l|}{0} \\
\hline Energy: & \multicolumn{2}{|l|}{-476.429205} & a.u \\
\hline Gibbs: & \multicolumn{2}{|l|}{-476.473704} & a.u \\
\hline Multiplicity: & \multicolumn{2}{|l|}{1} & \\
\hline Dipole: & \multicolumn{2}{|l|}{7.5778} & \\
\hline Setup: & \multicolumn{3}{|c|}{ B3LYP, def2TZVP } \\
\hline Formula: & \multicolumn{3}{|c|}{ C5H9NO3 } \\
\hline Charge: & \multicolumn{3}{|c|}{0} \\
\hline Energy: & \multicolumn{3}{|c|}{-476.445840 a.u } \\
\hline Gibbs: & \multicolumn{3}{|c|}{-476.490287 a.u } \\
\hline Multiplicity: & \multicolumn{3}{|c|}{1} \\
\hline Dipole: & \multicolumn{3}{|l|}{7.7377} \\
\hline Setup: & \multicolumn{3}{|l|}{ M06, def2TZVP } \\
\hline Formula: & \multicolumn{3}{|l|}{ C5H9NO3 } \\
\hline Charge: & \multicolumn{3}{|l|}{0} \\
\hline Energy: & -476.144590 & a.u & \\
\hline Gibbs: & -476.189240 & a.u & \\
\hline Multiplicity: & 1 & & \\
\hline Dipole: & 7.1614 & & \\
\hline
\end{tabular}

$\begin{array}{lrll}\text { C, } & -1.315555, & 5.825191, & 1.483916 \\ \text { C, } & 0.016938, & 6.139128, & 2.170302 \\ \text { C, } & -1.226030, & 8.064169, & 2.109627 \\ \text { H, } & -1.176143, & 5.310360, & 0.535727 \\ \text { H, } & -1.982370, & 5.228565, & 2.112581 \\ \text { H, } & 0.843530, & 6.168287, & 1.461389 \\ \text { H, } & 0.254879, & 5.464759, & 2.986868 \\ \text { N, } & -1.847155, & 7.167327, & 1.301778 \\ \text { O, } & -0.158538, & 7.470724, & 2.712976 \\ \text { O, } & -1.521772, & 9.223366, & 2.311833\end{array}$




$\begin{array}{lllr}\text { C, } & -3.119311, & 7.432241, & 0.664320 \\ \text { H, } & -3.841550, & 6.667753, & 0.961430 \\ \text { H, } & -3.480380, & 8.395799, & 1.018702 \\ \text { C, } & -3.001576, & 7.455922, & -0.853827 \\ \text { H, } & -2.320147, & 8.259069, & -1.152426 \\ \text { H, } & -2.586712, & 6.508292, & -1.212820 \\ \text { O, } & -4.315246, & 7.667857, & -1.364026 \\ \text { H, } & -4.273960, & 7.673396, & -2.324982\end{array}$




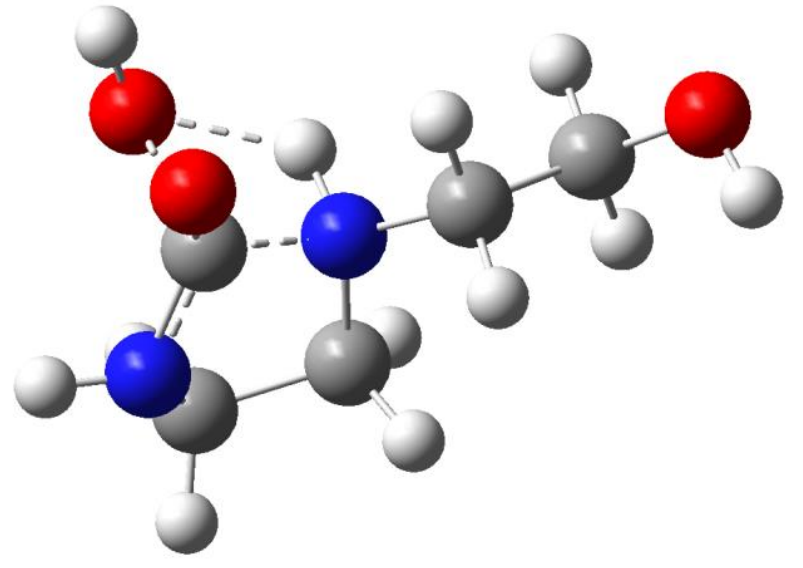

Formula:

Charge:

Energy:

Gibbs:

Multiplicity:

Dipole:

Setup:

Formula:

Charge:

Energy:

Gibbs:

Multiplicity:

Dipole:

\section{Setup:}

Formula:

Charge:

Energy:

Gibbs:

Multiplicity:

Dipole:
$\mathrm{C} 5 \mathrm{H} 12 \mathrm{~N} 2 \mathrm{O} 3$

$$
0
$$

$-532.924822$

$-532.971378$

1

8.3006

B3LYP, def2TZVP

C5H12N2O3

0

-532.942317 a.u

-532.989015 a.u

1

8.6594

M06, def2TZVP

C5H12N2O3

0

-532.589279 a.u -532.636288 a.u

1

8.4621

$\begin{array}{lrrr}\text { C, } & -0.315739, & 1.146642, & 0.071421 \\ \text { C, } & 2.117824, & 0.465795, & 0.552183 \\ \text { H, } & 1.883381, & -0.155206, & 1.416854 \\ \text { H, } & 2.184549, & 1.500593, & 0.879808 \\ \text { C, } & 3.432289, & 0.035457, & -0.091808 \\ \text { H, } & 3.630631, & 0.654994, & -0.967260 \\ \text { H, } & 3.381595, & -1.005215, & -0.423308 \\ \text { O, } & 4.516779, & 0.239399, & 0.803780 \\ \text { H, } & 4.485718, & -0.438385, & 1.487301 \\ \text { O, } & -0.177730, & 1.951963, & -1.798971 \\ \text { C, } & 0.538562, & -0.972803, & -0.813446 \\ \text { H, } & 0.699664, & -1.651502, & 0.020842\end{array}$




$\begin{array}{lrrr}\text { H, } & 1.096239, & -1.316657, & -1.678716 \\ \text { C, } & -0.953507, & -0.782918, & -1.084981 \\ \text { H, } & -1.503947, & -1.708195, & -0.938408 \\ \text { H, } & -1.124296, & -0.405033, & -2.094106 \\ \text { N, } & -1.308531, & 0.205008, & -0.076093 \\ \text { O, } & -0.251866, & 2.057950, & 0.865273 \\ \text { N, } & 0.993344, & 0.383979, & -0.399257 \\ \text { H, } & 1.099194, & 1.002069, & -1.243902 \\ \text { H, } & -0.116255, & 2.875985, & -1.538873 \\ \text { H, } & -2.243756, & 0.581350, & -0.092622\end{array}$




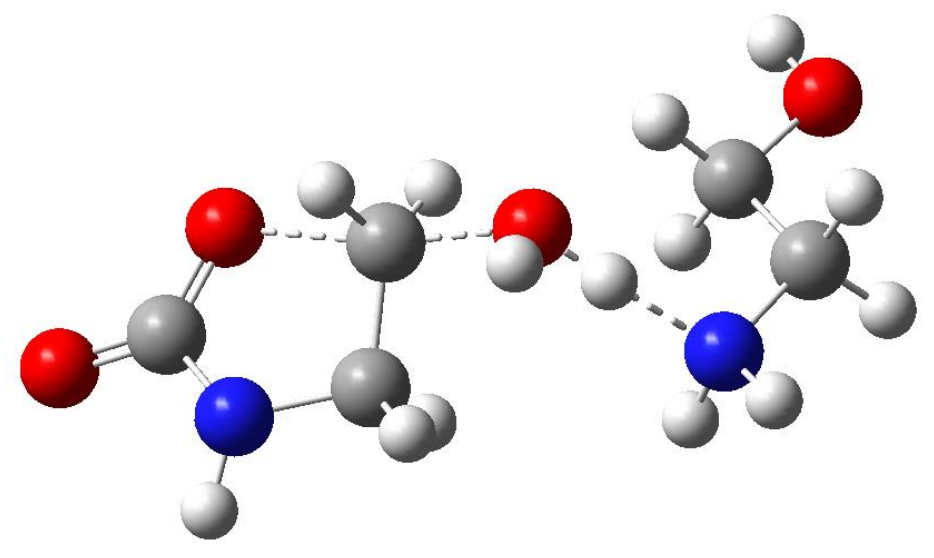

Formula:

Charge:

Energy:

Gibbs:

Multiplicity:

Dipole:

\section{Setup:}

Formula:

Charge:

Energy:

Gibbs:

Multiplicity:

Dipole:

Setup:

Formula:

Charge:

Energy:

Gibbs:

Multiplicity:

Dipole:
C5H14N2O4

0

-609.375575 a.u

$-609.432866 \quad$ a.u

1

16.9188

B3LYP, def2TZVP

C5H14N2O4

0

-609.395669 a.u

-609.453063 a.u

1

17.1599

M06, def2TZVP

0

a.u

a.u

1

$\begin{array}{lrrr}\text { C, } & 0.934440, & -0.192568, & -0.023130 \\ \text { H, } & 1.455991, & 0.251575, & -0.878363 \\ \text { H, } & 1.154104, & -1.258880, & -0.016494 \\ \text { C, } & 1.441139, & 0.446247, & 1.249473 \\ \text { H, } & 2.078740, & 1.307157, & 1.220778 \\ \text { H, } & 0.944566, & 0.226677, & 2.177142 \\ \text { N, } & -0.502151, & -0.009824, & -0.082933 \\ \text { H, } & -0.982653, & -0.434533, & -0.860116 \\ \text { C, } & -0.948408, & 1.265465, & 0.291067 \\ \text { O, } & -2.085250, & 1.645254, & -0.030956 \\ \text { O, } & -0.085928, & 1.903830, & 0.990742\end{array}$




$\begin{array}{lllr}\text { H, } & 2.608323, & -1.532050, & 1.848646 \\ \text { O, } & 2.902961, & -0.632155, & 1.658366 \\ \text { C, } & 5.149529, & 1.675107, & 0.233003 \\ \text { H, } & 4.539609, & 1.630922, & 1.141252 \\ \text { H, } & 4.512142, & 2.029856, & -0.585726 \\ \text { C, } & 5.684592, & 0.291692, & -0.094099 \\ \text { H, } & 6.360617, & 0.361533, & -0.949333 \\ \text { H, } & 6.255226, & -0.084270, & 0.754506 \\ \text { O, } & 6.276633, & 2.527030, & 0.413452 \\ \text { H, } & 5.961394, & 3.407769, & 0.637773 \\ \text { H, } & 4.923724, & -1.579656, & -0.488545 \\ \text { H, } & 3.561960, & -0.677023, & 0.836919 \\ \text { H, } & 4.088674, & -0.389088, & -1.206937 \\ \text { N, } & 4.567780, & -0.640479, & -0.347735\end{array}$




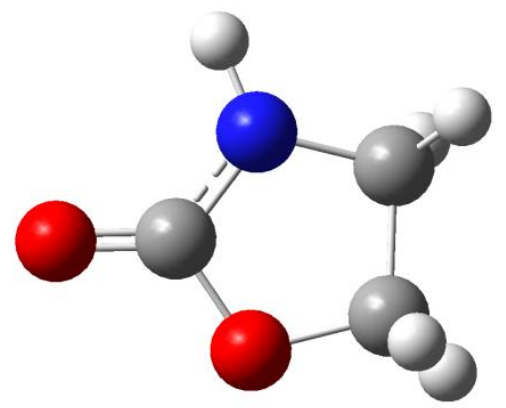

\begin{tabular}{|c|c|c|}
\hline Formula: & $\mathrm{C} 3 \mathrm{H} 5 \mathrm{NO} 2$ & \\
\hline Charge: & 0 & \\
\hline Energy: & -322.590256 & a.u \\
\hline Gibbs: & -322.623839 & a.u \\
\hline Multiplicity: & 1 & \\
\hline Dipole: & 6.8510 & \\
\hline Setup: & B3LYP, def2TZVP & \\
\hline Formula: & $\mathrm{C} 3 \mathrm{H} 5 \mathrm{NO} 2$ & \\
\hline Charge: & 0 & \\
\hline Energy: & -322.602419 a.u & \\
\hline Gibbs: & -322.635983 a.u & \\
\hline Multiplicity: & 1 & \\
\hline Dipole: & 7.0451 & \\
\hline Setup: & M06, def2TZVP & \\
\hline Formula: & C3H5NO2 & \\
\hline Charge: & 0 & \\
\hline Energy: & -322.404114 a.u & \\
\hline Gibbs: & $-322.438649 \quad a . u$ & \\
\hline $\begin{array}{l}\text { Multiplicity: } \\
\text { Dipole: }\end{array}$ & $\begin{array}{l}1 \\
6.7281\end{array}$ & \\
\hline
\end{tabular}

$\begin{array}{llll}\text { C, } & -1.440154, & 5.730298, & 1.889827 \\ \text { C, } & -0.007951, & 6.081514, & 2.312499 \\ \text { C, } & -0.964859, & 7.959363, & 1.409642 \\ \text { H, } & -1.481908, & 4.812012, & 1.309032 \\ \text { H, } & -2.110877, & 5.641602, & 2.746940 \\ \text { H, } & 0.732123, & 5.649936, & 1.639211 \\ \text { H, } & 0.222187, & 5.810020, & 3.337881 \\ \text { N, } & -1.753110, & 6.900864, & 1.083324 \\ \text { H, } & -2.674539, & 7.076640, & 0.720606 \\ \text { O, } & 0.060979, & 7.524473, & 2.193612 \\ \text { O, } & -1.086704, & 9.119352, & 1.082646\end{array}$



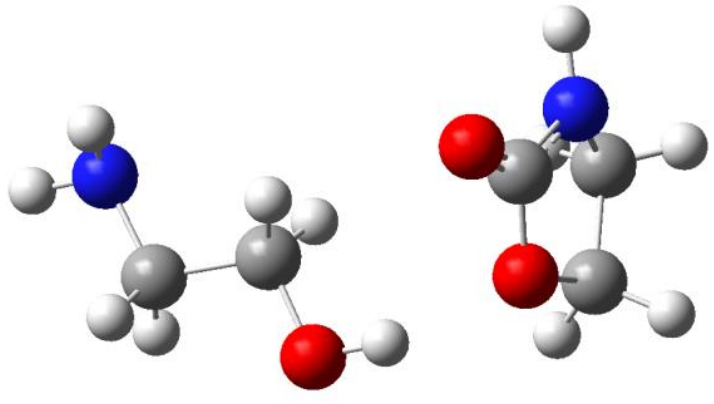

Formula:

Charge:

Energy:

Gibbs:

Multiplicity:

Dipole:

Setup:

Formula:

Charge:

Energy :

Gibbs:

Multiplicity:

Dipole:

\section{Setup:}

Formula:

Charge:

Energy:

Gibbs:

Multiplicity

Dipole:
$\mathrm{C} 5 \mathrm{H} 12 \mathrm{~N} 2 \mathrm{O} 3$

0

-532.977372 a.u

-533.032062 a.u

1

6.4415

B3LYP, def2TZVP

$\mathrm{C} 5 \mathrm{H} 12 \mathrm{~N} 2 \mathrm{O} 3$

0 $-532.994456 \quad$ a.u

a -533.048858 .u

1

6.8764

M06, def2TZVP

$\mathrm{C} 5 \mathrm{H} 12 \mathrm{~N} 2 \mathrm{O} 3$

0

-532.648106 a.u -532.701038 a.u

1

6.9237

$\begin{array}{llll}\text { C, } & 10.504567, & -7.546756, & 12.721673 \\ \text { H, } & 11.418181, & -8.131689, & 12.691994 \\ \text { H, } & 10.640150, & -6.672019, & 13.355130 \\ \text { C, } & 9.964350, & -7.178862, & 11.333534 \\ \text { H, } & 10.192381, & -6.149992, & 11.066871 \\ \text { H, } & 10.345218, & -7.843274, & 10.555962 \\ \text { C, } & 8.294425, & -8.196086, & 12.605710 \\ \text { O, } & 9.459899, & -8.366710, & 13.309972 \\ \text { H, } & 7.859758, & -7.314487, & 10.815786 \\ \text { N, } & 8.539742, & -7.380210, & 11.553770 \\ \text { O, } & 7.259283, & -8.722111, & 12.943161 \\ \text { C, } & 9.963653, & -12.938018, & 11.803066 \\ \text { H, } & 9.603094, & -13.560107, & 12.632307 \\ \text { H, } & 11.049727, & -13.044553, & 11.770734 \\ \text { C, } & 9.627175, & -11.489137, & 12.107003 \\ \text { H, } & 10.038207, & -10.848980, & 11.318139\end{array}$




$\begin{array}{llll}\text { H, } & 8.538362, & -11.353885, & 12.107458 \\ \text { O, } & 10.177226, & -11.158706, & 13.380459 \\ \text { H, } & 9.937321, & -10.241572, & 13.568569 \\ \text { N, } & 9.418816, & -13.316055, & 10.493793 \\ \text { H, } & 9.737862, & -14.244587, & 10.245075 \\ \text { H, } & 8.407868, & -13.376999, & 10.549032\end{array}$




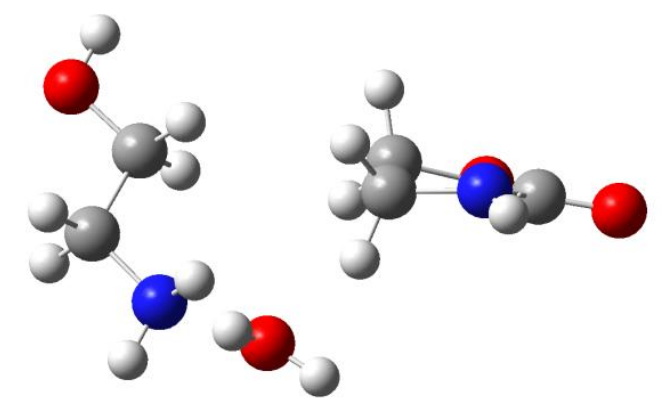

Formula:

Charge:

Energy:

Gibbs:

Multiplicity:

Dipole:

Setup:

Formula:

Charge:

Energy:

Gibbs:

Multiplicity:

Dipole:

Setup:

Formula:

Charge:

Energy:

Gibbs:

Multiplicity:

Dipole:
C5H14N2O4

0

$-609.428541 \quad$ a.u

$-609.490056 \quad$ a.u

1

8.6927

B3LYP, def2TZVP

C5H14N2O4

0

-609.447738 a.u

-609.510230 a.u

1

8.8742

M06, def2TZVP

C5H14N2O4

0

-609.059067 a.u

-609.119745 a.u

1

8.7566

$\begin{array}{lllr}\text { C, } & -2.728548, & -1.186021, & -0.449131 \\ \text { H, } & -3.247886, & -1.848417, & -1.137816 \\ \text { H, } & -3.155605, & -0.184544, & -0.526414 \\ \text { C, } & -2.764996, & -1.704844, & 0.994275 \\ \text { H, } & -2.913483, & -2.783169, & 1.038484 \\ \text { H, } & -3.496979, & -1.187651, & 1.605235 \\ \text { N, } & -1.292783, & -1.179575, & -0.681253 \\ \text { H, } & -0.879108, & -0.775517, & -1.503999 \\ \text { C, } & -0.595289, & -1.151144, & 0.485292 \\ \text { O, } & 0.588149, & -0.947871, & 0.649736 \\ \text { O, } & -1.443226, & -1.410174, & 1.517319 \\ \text { H, } & -3.918556, & 1.422778, & 1.539816 \\ \text { O, } & -4.706843, & 0.909521, & 1.740606 \\ \text { C, } & -6.460095, & -1.852019, & 0.139372 \\ \text { H, } & -5.932438, & -1.614324, & 1.067990 \\ \text { H, } & -5.723839, & -2.226289, & -0.582313\end{array}$




$\begin{array}{lllr}\text { C, } & -7.121500, & -0.598629, & -0.406788 \\ \text { H, } & -7.704622, & -0.864615, & -1.294426 \\ \text { H, } & -7.816930, & -0.213242, & 0.339657 \\ \text { O, } & -7.487581, & -2.816704, & 0.360103 \\ \text { H, } & -7.086439, & -3.610635, & 0.726163 \\ \text { H, } & -6.559044, & 1.302360, & -0.937567 \\ \text { H, } & -5.153423, & 0.778035, & 0.869505 \\ \text { H, } & -5.530715, & 0.165657, & -1.460564 \\ \text { N, } & -6.108297, & 0.434873, & -0.671519\end{array}$




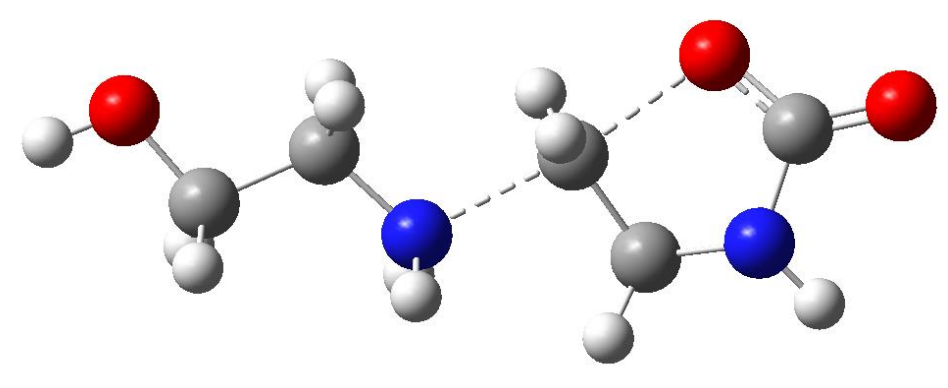

Formula:

Charge:

Energy:

Gibbs:

Multiplicity:

Dipole:

Setup:

Formula:

Charge:

Energy:

Gibbs:

Multiplicity:

Dipole:

Setup:

Formula:

Charge:

Energy:

Gibbs:

Multiplicity:

Dipole:
$\mathrm{C} 5 \mathrm{H} 12 \mathrm{~N} 2 \mathrm{O} 3$

0

-532.933919 a.u

-532.983953 a.u

1

17.3753

B3LYP, def2TZVP

C5H12N2O3

0

-532.951970 a.u

-533.002064 a.u

1

17.6218

M06, def2TZVP

C5H12N2O3

0

-532.598441 a.u

-532.648500 a.u

1

17.3223

$\begin{array}{llll}\text { C, } & 10.441561, & -7.419143, & 12.509922 \\ \text { H, } & 10.882030, & -8.337635, & 12.176387 \\ \text { H, } & 10.539371, & -7.151641, & 13.546614 \\ \text { C, } & 9.572562, & -6.579179, & 11.598096 \\ \text { H, } & 9.946703, & -5.558365, & 11.544467 \\ \text { H, } & 9.599260, & -7.001310, & 10.587686 \\ \text { C, } & 7.844606, & -7.763733, & 12.749799 \\ \text { O, } & 8.849999, & -8.525811, & 13.015140 \\ \text { H, } & 7.524241, & -6.052205, & 11.665927 \\ \text { N, } & 8.236655, & -6.562570, & 12.162175 \\ \text { O, } & 6.654338, & -7.998183, & 12.994509 \\ \text { C, } & 14.671994, & -6.602988, & 12.521051 \\ \text { H, } & 14.674703, & -5.587760, & 12.933258 \\ \text { H, } & 14.875516, & -6.532061, & 11.446821 \\ \text { C, } & 13.311057, & -7.245012, & 12.744424 \\ \text { H, } & 13.315473, & -8.257563, & 12.341216\end{array}$




$\begin{array}{llll}\text { H, } & 13.109998, & -7.314376, & 13.813315 \\ \text { N, } & 12.209427, & -6.504843, & 12.117013 \\ \text { H, } & 12.151430, & -5.555697, & 12.471864 \\ \text { H, } & 12.327178, & -6.449239, & 11.110194 \\ \text { O, } & 15.629475, & -7.429602, & 13.176075 \\ \text { H, } & 16.502457, & -7.044514, & 13.052356\end{array}$




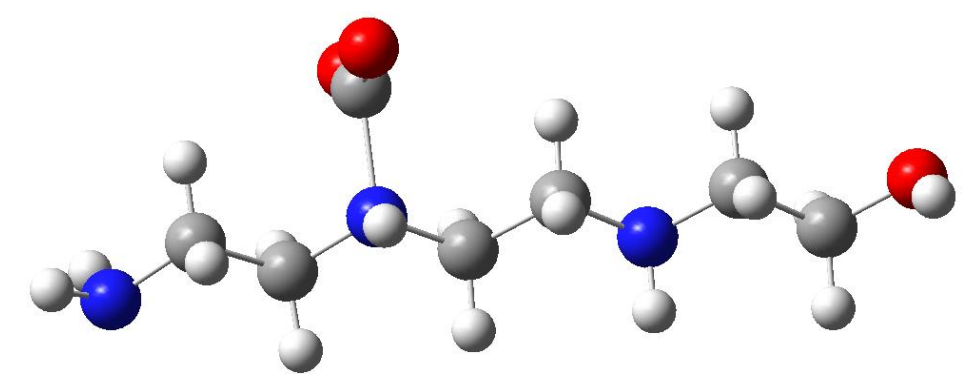

Formula:

Charge:

C7H17N303

Energy:

0

Gibbs:

$-666.923961$

a.u

Multiplicity:

$-666.985782$

a.u

Dipole:

8.2142

Setup:

Formula:

Charge:

B3LYP, def2TZVP

C7H17N303

0

Energy:

-666.943465 a.u

Gibbs:

-667.004676 a.u

Multiplicity: 1

Dipole:

8.6933

Setup:

Formula:

M06, def2TZVP

Charge:

C7H17N303

Energy:

0

Gibbs:

-666.466979 a.u -666.525151 a.u

Multiplicity: $\quad 1$

Dipole:

10.0493

$\begin{array}{lrrr}\text { C, } & -1.557864, & 1.431929, & 0.295473 \\ \text { C, } & 1.449502, & -2.908945, & 0.164549 \\ \text { H, } & 1.511126, & -3.138384, & 1.240615 \\ \text { H, } & 2.073376, & -2.029684, & -0.009465 \\ \text { C, } & 2.019626, & -4.079218, & -0.626743 \\ \text { H, } & 2.040905, & -3.828952, & -1.687330 \\ \text { H, } & 1.379072, & -4.961108, & -0.506621 \\ \text { O, } & 3.365158, & -4.372084, & -0.254531 \\ \text { H, } & 3.365944, & -4.691399, & 0.653939 \\ \text { C, } & -4.621187, & 1.291521, & 0.804695 \\ \text { H, } & -4.026476, & 2.211934, & 0.776985 \\ \text { H, } & -4.698125, & 0.991205, & 1.851679 \\ \text { O, } & -1.151719, & 1.781332, & 1.378101 \\ \text { C, } & -0.498525, & -1.466935, & 0.414317 \\ \text { H, } & 0.161674, & -0.609254, & 0.273210 \\ \text { H, } & -0.591791, & -1.619941, & 1.500965 \\ \text { C, } & -3.910419, & 0.197507, & 0.018144\end{array}$




$\begin{array}{lrrr}\text { H, } & -4.471781, & -0.734598, & 0.089306 \\ \text { H, } & -3.830492, & 0.472654, & -1.033052 \\ \text { N, } & -5.976192, & 1.452038, & 0.271143 \\ \text { H, } & -6.509268, & 2.068454, & 0.872466 \\ \text { H, } & -5.937449, & 1.909087, & -0.633417 \\ \text { C, } & -1.867627, & -1.166017, & -0.180829 \\ \text { H, } & -2.515676, & -2.041359, & -0.104453 \\ \text { H, } & -1.773289, & -0.899175, & -1.231260 \\ \text { N, } & -2.527841, & -0.027918, & 0.501086 \\ \text { O, } & -1.531399, & 1.721804, & -0.875306 \\ \text { N, } & 0.088946, & -2.609655, & -0.271968 \\ \text { H, } & -0.494727, & -3.425316, & -0.119049 \\ \text { H, } & -2.533536, & -0.185557, & 1.506246\end{array}$




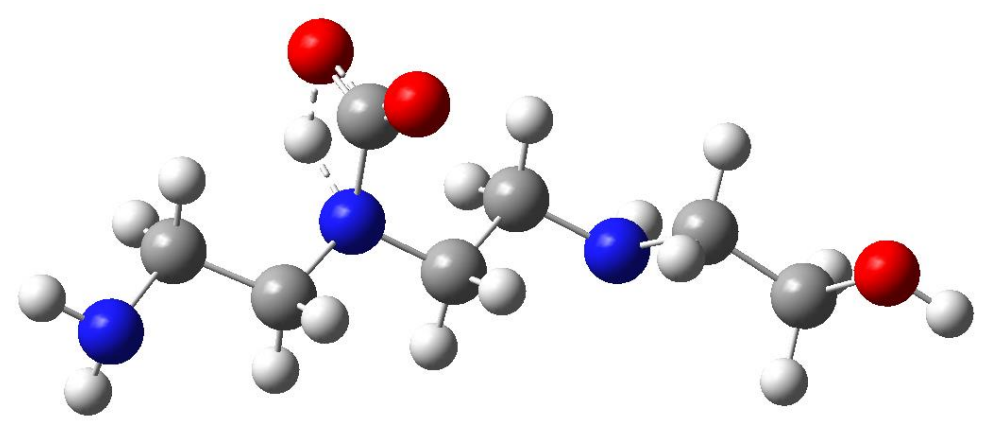

\begin{tabular}{|c|c|c|}
\hline Formula: & \multicolumn{2}{|l|}{ C7H17N303 } \\
\hline Charge: & \multicolumn{2}{|l|}{0} \\
\hline Energy: & \multicolumn{2}{|l|}{-666.876506} \\
\hline Gibbs: & \multicolumn{2}{|l|}{-666.936889} \\
\hline Multiplicity: & \multicolumn{2}{|l|}{1} \\
\hline Dipole: & \multicolumn{2}{|l|}{7.6043} \\
\hline $\begin{array}{l}\text { Setup: } \\
\text { Formula: }\end{array}$ & \multicolumn{2}{|c|}{ B3LYP, def2TZVP } \\
\hline Charge: & \multicolumn{2}{|l|}{0} \\
\hline Energy: & -666.895892 & a.u \\
\hline Gibbs: & -666.956123 & a.u \\
\hline Multiplicity: & \multicolumn{2}{|c|}{1} \\
\hline Dipole: & \multicolumn{2}{|l|}{7.8257} \\
\hline Setup: & \multicolumn{2}{|c|}{ M06, def2TZVP } \\
\hline Formula: & \multicolumn{2}{|c|}{ C7H17N303 } \\
\hline Charge: & \multicolumn{2}{|l|}{0} \\
\hline Energy: & -666.445040 & a.u \\
\hline Gibbs: & -666.505123 & a.u \\
\hline Multiplicity: & 1 & \\
\hline Dipole: & 7.6213 & \\
\hline
\end{tabular}

$\begin{array}{rrrr}\text { C, } & -1.419780, & 1.483922, & -0.465556 \\ \text { H, } & -1.360509, & 2.573964, & -0.337125 \\ \text { H, } & -1.619425, & 1.297647, & -1.521191 \\ \text { C, } & -2.575236, & 0.917902, & 0.356851 \\ \text { H, } & -2.690379, & -0.140496, & 0.131978 \\ \text { H, } & -2.378360, & 1.017497, & 1.427175 \\ \text { N, } & -3.859570, & 1.585967, & 0.053212 \\ \text { H, } & -4.031878, & 2.290215, & -1.074624 \\ \text { C, } & -5.011462, & 0.913110, & 0.693046 \\ \text { H, } & -4.917516, & 0.995182, & 1.778677 \\ \text { H, } & -4.954605, & -0.142471, & 0.427626 \\ \text { C, } & -6.349343, & 1.487026, & 0.239594 \\ \text { H, } & -6.395809, & 1.464045, & -0.856346 \\ \text { H, } & -6.425365, & 2.532892, & 0.542023 \\ \text { N, } & -7.429577, & 0.747214, & 0.896272 \\ \text { H, } & -8.314661, & 1.207907, & 0.723222\end{array}$




$\begin{array}{lrrr}\text { H, } & -7.511375, & -0.178287, & 0.489923 \\ \text { C, } & -3.829100, & 3.076306, & 0.333391 \\ \text { O, } & -4.018960, & 3.525691, & -0.865417 \\ \text { O, } & -3.668620, & 3.598842, & 1.403173 \\ \text { N, } & -0.170990, & 0.820552, & -0.100554 \\ \text { H, } & 0.454380, & 0.837690, & -0.895721 \\ \text { C, } & 0.503927, & 1.412670, & 1.052460 \\ \text { H, } & 0.810178, & 2.453998, & 0.875247 \\ \text { H, } & -0.181844, & 1.424049, & 1.901103 \\ \text { C, } & 1.724590, & 0.593631, & 1.430748 \\ \text { H, } & 2.415380, & 0.541492, & 0.580462 \\ \text { H, } & 1.416734, & -0.427860, & 1.675473 \\ \text { O, } & 2.342232, & 1.232977, & 2.548258 \\ \text { H, } & 3.114109, & 0.719294, & 2.804013\end{array}$




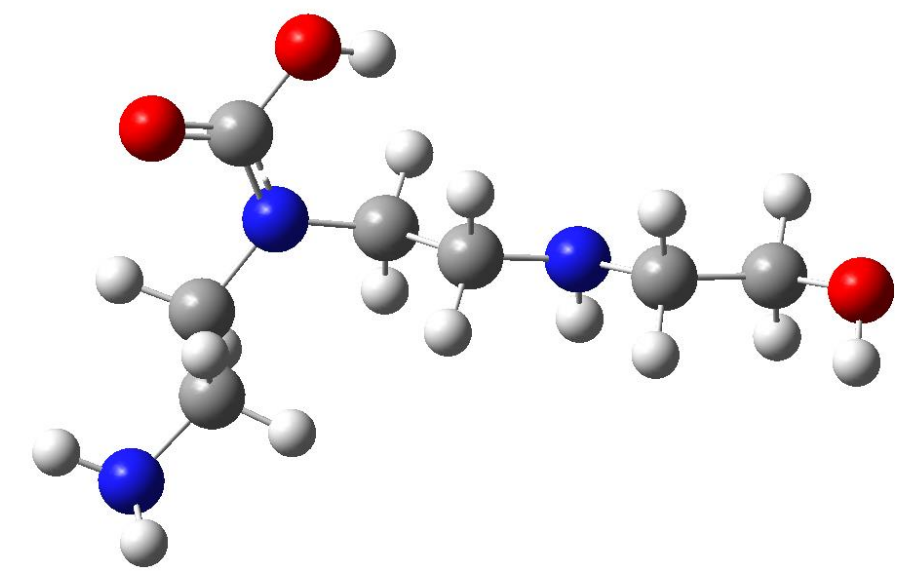

Formula:

Charge:

Energy:

Gibbs:

Multiplicity:

Dipole:

Setup:

Formula:

Charge:

Energy:

Gibbs:

Multiplicity:

Dipole:

Setup:

Formula:

Charge:

Energy:

Gibbs:

Multiplicity:

Dipole:
C7H17N303

0

$-666.926615$

$-666.987725$

a.u

a.u

1

6.5986

B3LYP, def2TZVP

C7H17N303

0

-666.945760 a.u

-667.006681 a.u

1

6.7576

M06, def2TZVP

C7H17N303

0

-666.494493 a.u

-666.554691 a.u

1

6.4210

C,

-1.638031 ,

1.947381,

$-0.107592$

C, 0.727988,

-3.007649 ,

0.630190

$\mathrm{H}, \quad 0.079574$,

-3.447300 ,

1.405223

$\mathrm{H}, \quad 1.405547$,

-2.317336 ,

1.137320

C, 1.550037,

-4.115140,

$-0.016508$

H, 2.257359,

-3.681554 ,

$-0.723367$

$\mathrm{H}, \quad 0.893687$,

-4.791850 ,

$-0.576607$

0 2.325744,

-4.834550 ,

0.940539

$\mathrm{H}, \quad 1.723531$,

-5.309099 ,

1.523144

C, -4.123883,

0.109872 ,

0.962642

$\mathrm{H}, \quad-3.647323$,

0.692876 ,

1.759782

$\mathrm{H}, \quad-3.776407$,

-0.919255 ,

1.061666

$0, \quad-0.291298$,

2.058109,

$-0.267002$

C, -0.791689,

-1.158120 ,

0.167942 


$\begin{array}{llrr}\text { H, } & -0.108322, & -0.502679, & 0.711972 \\ \text { H, } & -1.557088, & -1.477512, & 0.888131 \\ \text { C, } & -3.674934, & 0.673756, & -0.385441 \\ \text { H, } & -4.041560, & 0.038856, & -1.191090 \\ \text { H, } & -4.102585, & 1.665057, & -0.521304 \\ \text { N, } & -5.588834, & 0.103682, & 1.020010 \\ \text { H, } & -5.899922, & -0.385171, & 1.850820 \\ \text { H, } & -5.932937, & 1.053604, & 1.110248 \\ \text { C, } & -1.459283, & -0.375888, & -0.966833 \\ \text { H, } & -2.150599, & -1.025335, & -1.501737 \\ \text { H, } & -0.705748, & -0.085906, & -1.704422 \\ \text { N, } & -2.220642, & 0.788730, & -0.517710 \\ \text { O, } & -2.240089, & 2.885180, & 0.384121 \\ \text { N, } & -0.021762, & -2.266933, & -0.379981 \\ \text { H, } & -0.651728, & -2.903511, & -0.857246 \\ \text { H, } & 0.091144, & 1.321652, & -0.758014\end{array}$




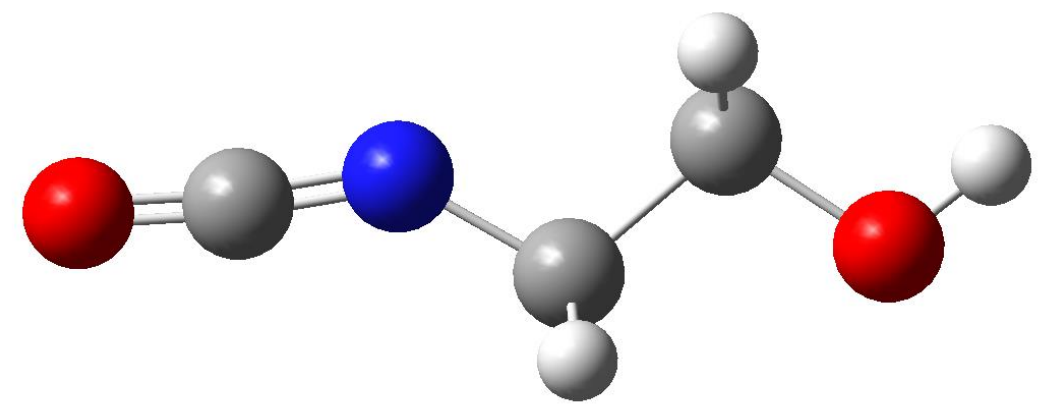

\begin{tabular}{|c|c|c|c|}
\hline & & $\mathrm{C} 3 \mathrm{H} 5 \mathrm{NO} 2$ & \\
\hline & & 0 & \\
\hline & & -322.565066 & a.u \\
\hline & & -322.604221 & a.u \\
\hline & city: & 1 & \\
\hline & & 3.5292 & \\
\hline & & B3LYP, def2TZVP & \\
\hline & & $\mathrm{C} 3 \mathrm{H} 5 \mathrm{NO} 2$ & \\
\hline & & 0 & \\
\hline & & -322.577098 a.u & \\
\hline & & -322.616214 a.u & \\
\hline & city: & 1 & \\
\hline & & 3.6070 & \\
\hline & & M06, def2TZVP & \\
\hline & & $\mathrm{C} 3 \mathrm{H} 5 \mathrm{NO} 2$ & \\
\hline & & 0 & \\
\hline & & -322.381443 a.u & \\
\hline & & -322.420656 a.u & \\
\hline & city: & 1 & \\
\hline & & 3.6605 & \\
\hline C, & -0.028150 & 0.455938, & -0.203989 \\
\hline$H$, & 0.176347 & -0.575397 & -0.494106 \\
\hline$H$, & 0.714894 & 0.757020 & 0.535522 \\
\hline$c$, & 0.074017 & 1.356969, & -1.423424 \\
\hline $\mathrm{H}$, & -0.669719 & 1.056124, & -2.167615 \\
\hline$H$, & -0.128994 & 2.392769, & -1.134681 \\
\hline N, & -1.353379 & 0.550607 & 0.366719 \\
\hline$C$, & -2.003460 & 0.116742 , & 1.267652 \\
\hline 0 , & -2.740901 & -0.238544 & 2.112722 \\
\hline 0 , & 1.405231, & 1.204412, & -1.922919 \\
\hline$H$, & 1.504409, & 1.765066, & -2.698157 \\
\hline
\end{tabular}




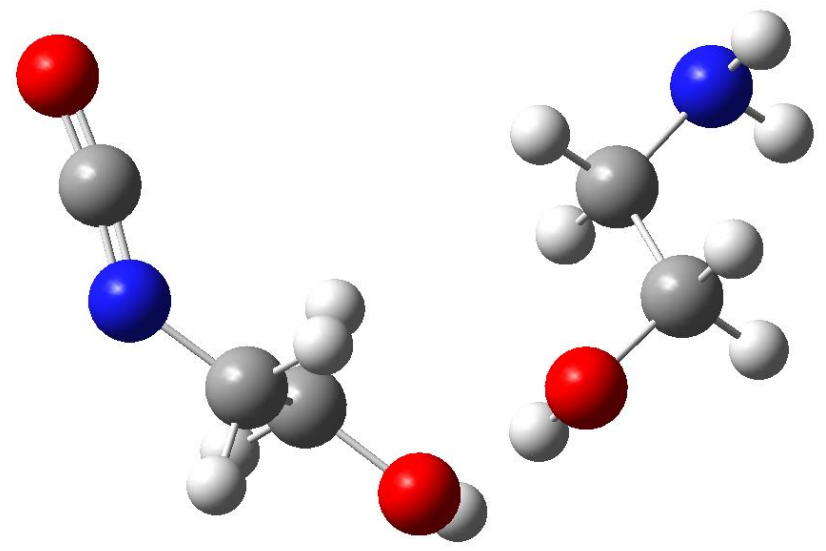

Formula:

Charge:

Energy:

Gibbs:

Multiplicity:

Dipole:

Setup:

Formula:

Charge:

Energy:

Gibbs:

Multiplicity:

Dipole:

Setup:

Formula:

Charge:

Energy:

Gibbs:

Multiplicity:

Dipole:
$\mathrm{C} 5 \mathrm{H} 12 \mathrm{~N} 2 \mathrm{O} 3$

0

$-532.957023 \quad$ a.u

-533.015122 a.u

1

4.8520

B3LYP, def2TZVP

C5H12N2O3

0

-532.973312 a.u

-533.032423 a.u

1

5.0166

M06, def2TZVP

$\mathrm{C} 5 \mathrm{H} 12 \mathrm{~N} 2 \mathrm{O} 3$

0

-532.627701 a.u

-532.685346 a.u

1

4.7735

$\begin{array}{lrrr}\text { C, } & -1.055691, & 0.468673, & 0.279366 \\ \text { H, } & -1.986822, & 0.086028, & 0.692619 \\ \text { H, } & -0.233306, & -0.099407, & 0.714742 \\ \text { C, } & -1.061154, & 0.288528, & -1.233967 \\ \text { H, } & -1.899439, & 0.832317, & -1.674453 \\ \text { H, } & -0.130728, & 0.679130, & -1.655251 \\ \text { N, } & -0.939613, & 1.863591, & 0.633847 \\ \text { C, } & -0.151500, & 2.759839, & 0.595316 \\ \text { O, } & 0.528866, & 3.718985, & 0.618013 \\ \text { O, } & -1.184508, & -1.116689, & -1.475427 \\ \text { H, } & -1.227582, & -1.271886, & -2.424618 \\ \text { C, } & 2.479123, & -1.247284, & -1.268078 \\ \text { H, } & 1.718891, & -0.856392, & -1.948715\end{array}$




$\begin{array}{lllr}\text { H, } & 2.576563, & -0.525185, & -0.454264 \\ \text { C, } & 1.992178, & -2.577677, & -0.691267 \\ \text { H, } & 1.879384, & -3.304662, & -1.506496 \\ \text { H, } & 2.743983, & -2.972763, & -0.004262 \\ \text { O, } & 0.785043, & -2.449275, & 0.053175 \\ \text { H, } & 0.096082, & -2.100259, & -0.538181 \\ \text { N, } & 3.751386, & -1.322112, & -1.991126 \\ \text { H, } & 4.475066, & -1.681752, & -1.377875 \\ \text { H, } & 3.675666, & -1.986600, & -2.753921\end{array}$




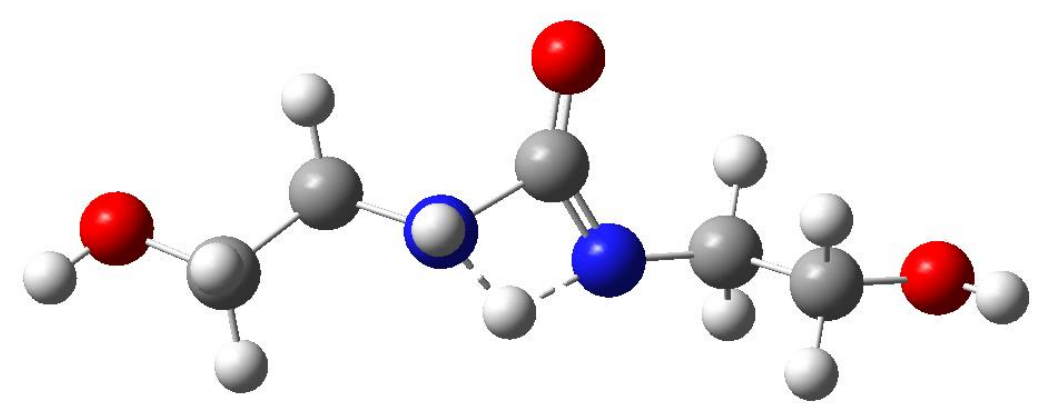

\begin{tabular}{|c|c|c|c|c|}
\hline & & $\mathrm{C} 5 \mathrm{H} 12 \mathrm{~N} 2 \mathrm{O} 3$ & & \\
\hline & & 0 & & \\
\hline & & -532.911212 & a.u & \\
\hline & & -532.963508 & a.u & \\
\hline & Lity: & 1 & & \\
\hline & & 7.4040 & & \\
\hline & & B3LYP, def2TZVP & & \\
\hline & & $\mathrm{C} 5 \mathrm{H} 12 \mathrm{~N} 2 \mathrm{O} 3$ & & \\
\hline & & 0 & & \\
\hline & & -532.928325 a.u & & \\
\hline & & $-532.980621 \quad a . u$ & & \\
\hline & city: & 1 & & \\
\hline & & 7.6366 & & \\
\hline & & M06, def2TZVP & & \\
\hline & & $\mathrm{C} 5 \mathrm{H} 12 \mathrm{~N} 2 \mathrm{O} 3$ & & \\
\hline & & $\theta$ & & \\
\hline & & -532.580259 a.u & & \\
\hline & & -532.632089 a.u & & \\
\hline & city: & 1 & & \\
\hline & & 7.4309 & & \\
\hline C, & 0.399745, & 0.289675 , & & 0.242921 \\
\hline$H$, & 0.324128 , & -0.523873 & & 0.970101 \\
\hline $\mathrm{H}$, & 1.201481, & 0.954616 , & & 0.568268 \\
\hline$C$, & 0.761173 , & -0.290294 & & -1.118386 \\
\hline$H$, & -0.021798 & -0.986621 & & -1.437095 \\
\hline $\mathrm{H}$, & 0.818357 & 0.516764 & & -1.856320 \\
\hline N, & -0.842689 & 1.030580, & & 0.190311 \\
\hline C, & -2.002541 & 0.506864 & & 0.546069 \\
\hline 0 , & -2.411528 & -0.545940 & & 1.001137 \\
\hline 0 , & 2.019378, & -0.955756 , & & -0.980618 \\
\hline $\mathrm{H}$, & 2.261095, & -1.329706 , & & -1.833238 \\
\hline$C$, & -3.593345 , & 2.278012, & & 1.404074 \\
\hline$H$, & -2.867440 & 2.466795, & & 2.194930 \\
\hline $\mathrm{H}$, & -4.315181 & 1.546295, & & 1.771393 \\
\hline$C$, & -4.300614 & 3.570276 , & & 1.036701 \\
\hline
\end{tabular}




$\begin{array}{lllr}\text { H, } & -3.567756, & 4.306417, & 0.691090 \\ \text { H, } & -5.008073, & 3.387769, & 0.219735 \\ \text { O, } & -4.973701, & 4.015988, & 2.209740 \\ \text { H, } & -5.427950, & 4.839417, & 2.006843 \\ \text { N, } & -2.867514, & 1.731506, & 0.248579 \\ \text { H, } & -3.492551, & 1.552956, & -0.533931 \\ \text { H, } & -1.654532, & 2.064764, & -0.048568\end{array}$




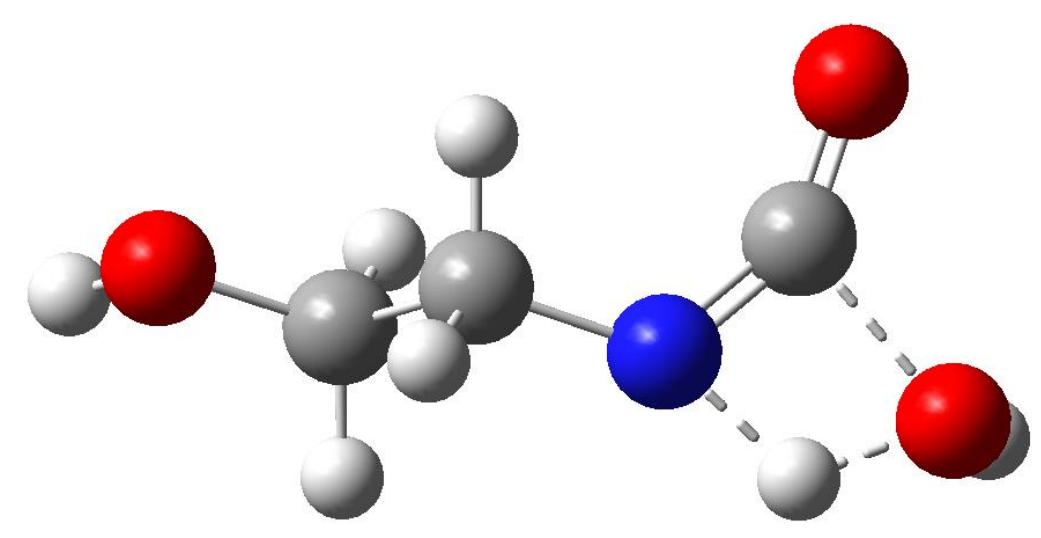

Formula:

Charge:

Energy:

Gibbs:

Multiplicity:

Dipole:

Setup:

Formula:

Charge:

Energy:

Gibbs:

Multiplicity:

Dipole:

Setup:

Formula:

Charge:

Energy:

Gibbs:

Multiplicity:

Dipole:
C3H7NO3

0

-398.953718 a.u

$-398.995821 \quad$ a.u

1

4.5916

B3LYP, def2TZVP

C3H7NO3

0

-398.967651 a.u

-399.009799 a.u

1

4.7204

M06, def2TZVP

$\mathrm{C} 3 \mathrm{H} 7 \mathrm{NO} 3$

0

-398.727671 a.u

-398.768808 a.u

1

4.7452

$\begin{array}{lrrr}\text { C, } & -0.320461, & 0.665305, & -1.168629 \\ \text { H, } & -0.564220, & 1.377817, & -1.959557 \\ \text { H, } & 0.301151, & -0.117963, & -1.603535 \\ \text { C, } & 0.462021, & 1.371172, & -0.070305 \\ \text { H, } & -0.143160, & 2.181787, & 0.348767 \\ \text { H, } & 0.685134, & 0.662256, & 0.733440 \\ \text { N, } & -1.530919, & 0.058680, & -0.636584 \\ \text { H, } & -2.235780, & -0.843522, & 0.040981 \\ \text { C, } & -2.708211, & 0.550238, & -0.834011 \\ \text { O, } & -3.383002, & 1.395864, & -1.319069 \\ \text { O, } & -3.432322, & -0.738238, & 0.007396 \\ \text { H, } & -3.806610, & -0.412431, & 0.838782 \\ \text { O, } & 1.659984, & 1.873573, & -0.668115 \\ \text { H, } & 2.178712, & 2.312152, & 0.013018\end{array}$




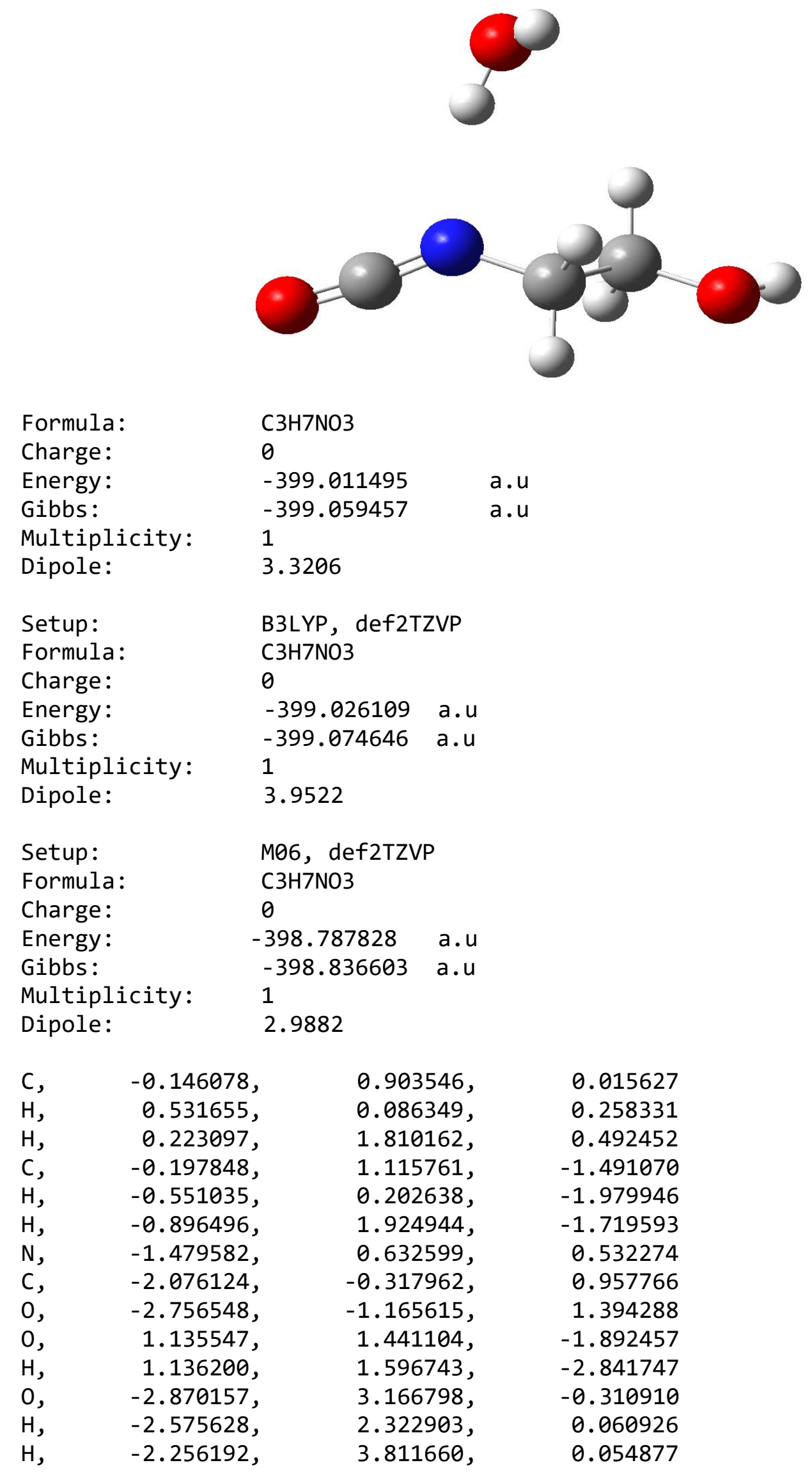




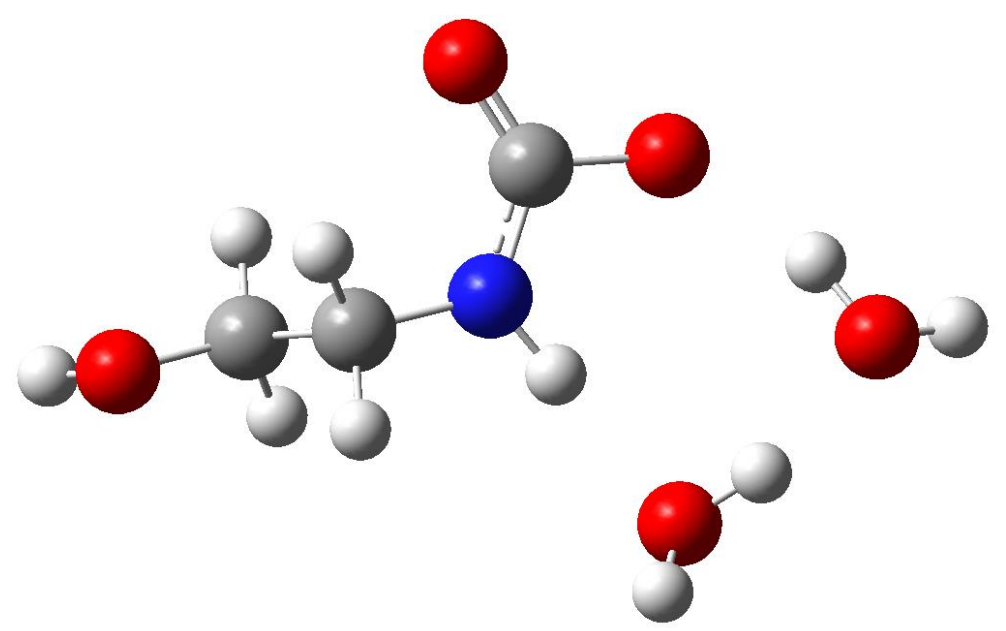

$\begin{array}{lll}\text { Formula: } & \text { C3H9NO4 } & \\ \text { Charge: } & 0 & \\ \text { Energy: } & -475.462917 & \text { a.u } \\ \text { Gibbs: } & -475.517923 & \text { a.u } \\ \text { Multiplicity: } & 1 & \\ \text { Dipole: } & 3.7519 & \\ & & \\ \text { Formula: } & \text { C3H9NO4 } & \\ \text { Charge: } & 0 & \\ \text { Energy: } & -475.478962 \mathrm{a} . \mathrm{u} & \\ \text { Gibbs: } & -475.534269 \mathrm{a} \cdot \mathrm{u} & \\ \text { Multiplicity: } & 1 & \\ \text { Dipole: } & 4.2547 & \\ & & \\ \text { Formula: } & \text { C3H9N04 } & \\ \text { Charge: } & 0 & \\ \text { Energy: } & -475.199019 & \text { a.u } \\ \text { Gibbs: } & -475.253683 & \text { a.u } \\ \text { Multiplicity: } & 1 & \\ \text { Dipole: } & 4.4349 & \end{array}$

$\begin{array}{lllr}\text { C, } & -1.625245, & 1.128767, & 0.055937 \\ \text { H, } & -0.868045, & 0.454181, & 0.450213 \\ \text { H, } & -1.888160, & 1.850279, & 0.828091 \\ \text { C, } & -1.079840, & 1.861166, & -1.163420 \\ \text { H, } & -0.797974, & 1.134438, & -1.932161 \\ \text { H, } & -1.858161, & 2.510341, & -1.574280 \\ \text { N, } & -2.795349, & 0.350051, & -0.289280 \\ \text { C, } & -3.920770, & 0.542228, & -0.637055 \\ \text { O, } & -5.043731, & 0.594905, & -0.989488 \\ \text { O, } & 0.047474, & 2.607954, & -0.712690 \\ \text { H, } & 0.414016, & 3.088301, & -1.461177\end{array}$




$\begin{array}{lllr}\text { O, } & -4.271010, & 3.282350, & -2.426663 \\ \text { H, } & -4.182577, & 3.400476, & -1.464778 \\ \text { H, } & -4.753455, & 2.453621, & -2.510317 \\ \text { O, } & -3.878411, & 3.406672, & 0.373909 \\ \text { H, } & -3.317320, & 4.129073, & 0.676338 \\ \text { H, } & -4.673406, & 3.461784, & 0.915292\end{array}$




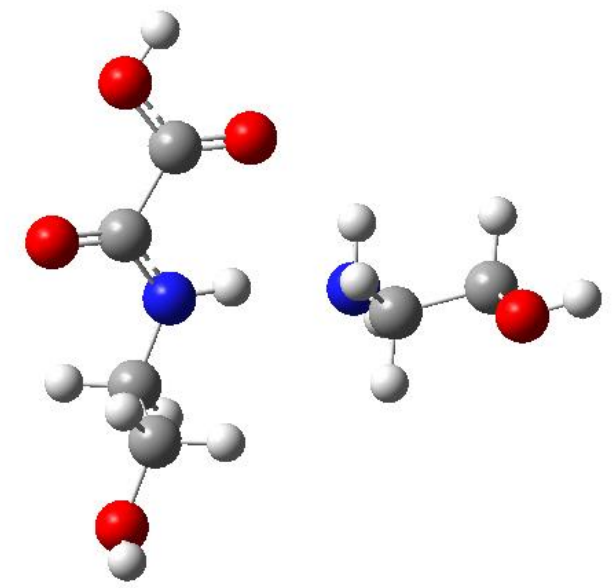

$\begin{array}{lll}\text { Formula: } & \text { C6H14N205 } & \\ \text { Charge: } & 0 & \\ \text { Energy : } & -722.778048 & \text { a.u } \\ \text { Gibbs: } & -722.843382 & \text { a.u }\end{array}$

Multiplicity: $\quad 1$

Dipole: $\quad 5.9645$

Setup: $\quad$ B3LYP, def2TZVP

Formula: $\quad$ C6H14N205

Charge: 0

Energy: $\quad-722.815197$ a.u

Gibbs: $\quad-722.880852$ a.u

Multiplicity: $\quad 1$

Dipole: $\quad 7.6302$

Setup: M06, def2TZVP

Formula: C6H14N205

Charge: 0

Energy: $\quad-722.366002$ a.u

Gibbs: $\quad-722.430954$ a.u

Multiplicity: $\quad 1$

Dipole: $\quad 7.4486$

$\begin{array}{llrr}\text { O, } & -3.568938, & 1.360524, & 0.058120 \\ \text { C, } & -6.219419, & -0.783921, & -0.588153 \\ \text { H, } & -5.602687, & -0.215644, & -1.285608 \\ \text { H, } & -6.320856, & -1.791041, & -0.996448 \\ \text { C, } & -7.596254, & -0.140142, & -0.509146 \\ \text { H, } & -7.496914, & 0.883793, & -0.127584 \\ \text { H, } & -8.224036, & -0.700891, & 0.195667 \\ \text { O, } & -8.153592, & -0.153244, & -1.820227 \\ \text { H, } & -9.013152, & 0.276264, & -1.798565 \\ \text { H, } & -6.024179, & -1.419698, & 1.363033 \\ \text { H, } & -5.381412, & 0.063403, & 1.082402 \\ \text { N, } & -5.511413, & -0.862204, & 0.690874\end{array}$




$\begin{array}{lllr}\text { C, } & -2.464635, & 1.117604, & -0.367958 \\ \text { O, } & -1.545704, & 2.049083, & -0.627219 \\ \text { H, } & -1.933570, & 2.910837, & -0.411096 \\ \text { C, } & -1.942324, & -0.300509, & -0.698173 \\ \text { O, } & -0.877451, & -0.463123, & -1.271084 \\ \text { N, } & -2.777369, & -1.266509, & -0.289977 \\ \text { H, } & -3.678172, & -1.038952, & 0.145683 \\ \text { C, } & -2.489487, & -2.666065, & -0.539041 \\ \text { H, } & -2.996887, & -3.262469, & 0.218484 \\ \text { H, } & -1.417109, & -2.835213, & -0.454859 \\ \text { C, } & -2.957362, & -3.101382, & -1.923063 \\ \text { H, } & -4.028868, & -2.894280, & -2.028573 \\ \text { H, } & -2.419254, & -2.523445, & -2.680447 \\ \text { O, } & -2.685755, & -4.495095, & -2.033790 \\ \text { H, } & -2.868281, & -4.775503, & -2.934680\end{array}$




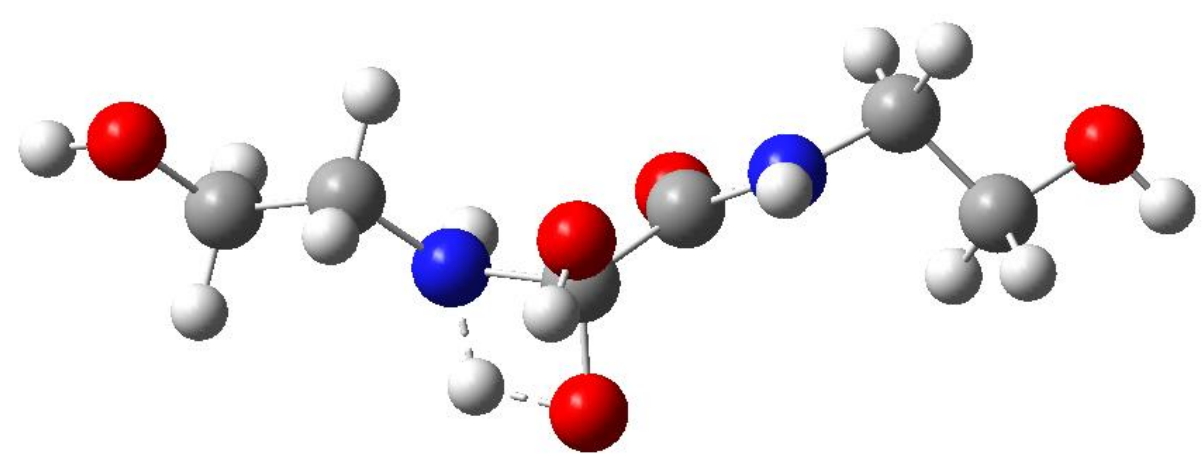

\begin{tabular}{|c|c|c|}
\hline Formula: & $\mathrm{C} 6 \mathrm{H} 14 \mathrm{~N} 2 \mathrm{O} 5$ & \\
\hline Charge: & 0 & \\
\hline Energy: & -722.731637 & a.u \\
\hline Gibbs: & -722.791383 & a.u \\
\hline Multiplicity: & 1 & \\
\hline Dipole: & 2.7565 & \\
\hline Setup: & \multicolumn{2}{|c|}{ B3LYP, def2TZVP } \\
\hline Formula: & \multicolumn{2}{|l|}{$\mathrm{C} 6 \mathrm{H} 14 \mathrm{~N} 2 \mathrm{O} 5$} \\
\hline Charge: & \multicolumn{2}{|l|}{0} \\
\hline Energy: & -722.756215 & a.u \\
\hline Gibbs: & -722.815797 & a.u \\
\hline Multiplicity: & \multicolumn{2}{|l|}{1} \\
\hline Dipole: & \multicolumn{2}{|l|}{2.8378} \\
\hline Setup: & \multicolumn{2}{|l|}{ M06, def2TZVP } \\
\hline Formula: & \multicolumn{2}{|l|}{$\mathrm{C} 6 \mathrm{H} 14 \mathrm{~N} 2 \mathrm{O} 5$} \\
\hline Charge: & \multicolumn{2}{|l|}{0} \\
\hline Energy: & -722.307227 & a.u \\
\hline Gibbs: & -722.366323 & a.u \\
\hline Multiplicity: & 1 & \\
\hline Dipole: & 2.9099 & \\
\hline
\end{tabular}

$\begin{array}{lrlr}\text { O, } & 1.304468, & 0.267834, & -0.240536 \\ \text { C, } & -0.359736, & 3.025939, & -1.279897 \\ \text { H, } & 0.576864, & 3.236698, & -1.790312 \\ \text { H, } & -1.135640, & 2.892484, & -2.035412 \\ \text { C, } & -0.716424, & 4.185911, & -0.366392 \\ \text { H, } & 0.066017, & 4.312766, & 0.388845 \\ \text { H, } & -1.658246, & 3.978246, & 0.153797 \\ \text { O, } & -0.831287, & 5.338073, & -1.195789 \\ \text { H, } & -1.050810, & 6.092689, & -0.640802 \\ \text { H, } & -1.048299, & 1.548666, & 0.010586 \\ \text { H, } & 0.822183, & 1.414027, & 0.153786 \\ \text { N, } & -0.198661, & 1.797190, & -0.494650 \\ \text { C, } & 0.331121, & 0.517658, & -1.157002 \\ \text { O, } & 0.790758, & 0.739798, & -2.459767\end{array}$




$\begin{array}{llll}\text { H, } & 1.726683, & 0.966774, & -2.379283 \\ \text { C, } & -0.786248, & -0.553331, & -1.173460 \\ \text { O, } & -1.666204, & -0.537388, & -0.314907 \\ \text { N, } & -0.663474, & -1.489555, & -2.122618 \\ \text { H, } & 0.084663, & -1.379353, & -2.787423 \\ \text { C, } & -1.540341, & -2.643800, & -2.213636 \\ \text { H, } & -2.531883, & -2.356592, & -1.867624 \\ \text { H, } & -1.613844, & -2.945399, & -3.257226 \\ \text { C, } & -1.029517, & -3.807320, & -1.373411 \\ \text { H, } & -0.954952, & -3.492121, & -0.327884 \\ \text { H, } & -0.030185, & -4.097455, & -1.714557 \\ \text { O, } & -1.962387, & -4.873484, & -1.530781 \\ \text { H, } & -1.664169, & -5.621657, & -1.004755\end{array}$




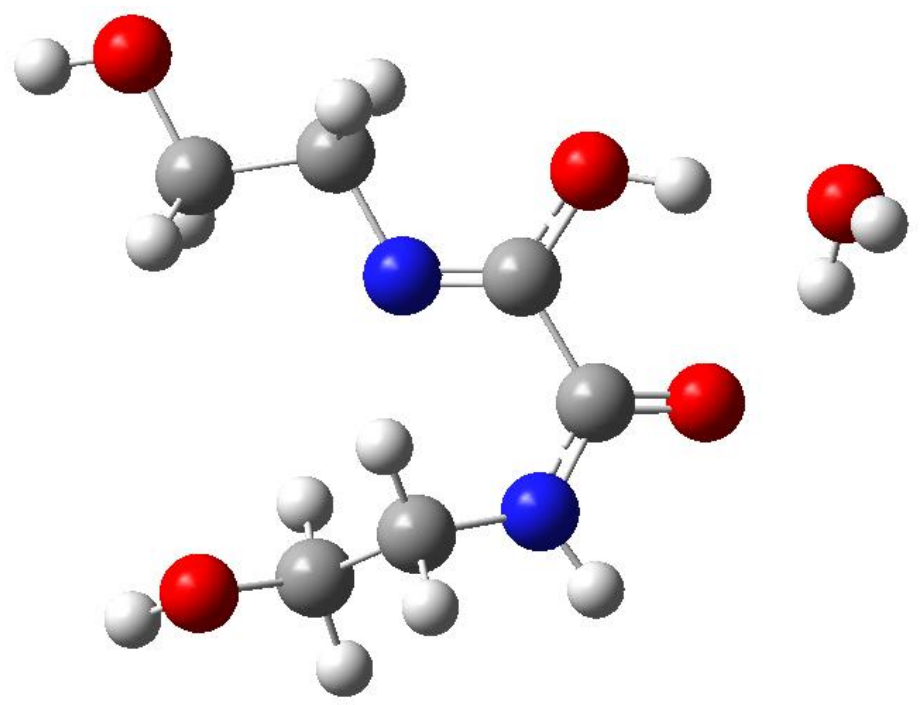

Formula:

Charge:

C6H14N2O5

Energy:

0

Gibbs:

$-722.778298$

a.u

$-722.841173$

a.u

Multiplicity:

1

Dipole:

5.6553

Formula:

C6H14N2O5

Charge:

Energy:

Gibbs:

$-722.801044$

a.u

-722.865086 a.u

Multiplicity: 1

Dipole:

2.9365

Formula:

C6H14N2O5

Charge:

0

Energy:

-722.347429 a.u

Gibbs:

-722.411208 a.u

Multiplicity: $\quad 1$

Dipole:

4. 5047

$\begin{array}{lrrr}\text { O, } & 3.097779, & -0.600507, & -2.360419 \\ \text { C, } & -0.840016, & 2.603406, & -0.560661 \\ \text { H, } & -0.093228, & 2.836311, & 0.205448 \\ \text { H, } & -0.495858, & 3.079497, & -1.484687 \\ \text { C, } & -2.181259, & 3.185398, & -0.153403 \\ \text { H, } & -2.522660, & 2.703621, & 0.769234 \\ \text { H, } & -2.921900, & 2.977053, & -0.933041 \\ \text { O, } & -2.003321, & 4.589782, & 0.028998 \\ \text { H, } & -2.847225, & 4.973137, & 0.285474 \\ \text { H, } & 3.636330, & -1.078769, & -1.720778 \\ \text { H, } & 2.322475, & -1.170404, & -2.526266 \\ \text { N, } & -0.973931, & 1.166272, & -0.731836 \\ \text { C, } & 0.045060, & 0.500189, & -1.076052\end{array}$




$\begin{array}{lrrr}\text { O, } & 1.247048, & 1.050557, & -1.292084 \\ \text { H, } & 1.933957, & 0.416133, & -1.619150 \\ \text { C, } & -0.090878, & -0.990859, & -1.346847 \\ \text { O, } & 0.544034, & -1.499122, & -2.281051 \\ \text { N, } & -0.909183, & -1.727514, & -0.582355 \\ \text { H, } & -0.959201, & -2.692302, & -0.878201 \\ \text { C, } & -1.687105, & -1.369076, & 0.597711 \\ \text { H, } & -1.627129, & -2.201479, & 1.299048 \\ \text { H, } & -1.253992, & -0.493864, & 1.069496 \\ \text { C, } & -3.143723, & -1.089150, & 0.257106 \\ \text { H, } & -3.183550, & -0.279627, & -0.473814 \\ \text { H, } & -3.603955, & -1.983275, & -0.177305 \\ \text { O, } & -3.782722, & -0.721886, & 1.479853 \\ \text { H, } & -4.706182, & -0.527150, & 1.294208\end{array}$


Formula: $\quad$ C6H14N205

Charge: $\quad 0$

Energy: $\quad-722.689644 \quad$ a.u

Gibbs: $\quad-722.750475$ a.u

Multiplicity: 1

Dipole: $\quad 7.7201$

$\begin{array}{lrrr}\text { O, } & 1.419091, & 0.717664, & -0.678971 \\ \mathrm{C}, & -0.667751, & 2.577039, & -1.012878 \\ \text { H, } & 0.102268, & 2.883390, & -0.287996 \\ \text { H, } & -0.340319, & 2.979839, & -1.985998 \\ \text { C, } & -1.963623, & 3.273753, & -0.638759 \\ \text { H, } & -2.300184, & 2.908434, & 0.338303 \\ \text { H, } & -2.738486, & 3.024984, & -1.372535 \\ \text { O, } & -1.729044, & 4.687195, & -0.603199 \\ \text { H, } & -2.554879, & 5.124746, & -0.377147 \\ \text { H, } & 2.045085, & 1.058337, & -1.326378 \\ \text { H, } & 1.406025, & -0.895483, & 0.119506 \\ \text { N, } & -0.888628, & 1.146819, & -1.035081 \\ \text { C, } & 0.186948, & 0.403585, & -1.333247 \\ \text { O, } & 0.535874, & 0.350323, & -2.799492 \\ \text { H, } & 1.265040, & -0.268875, & -2.944845 \\ \text { C, } & -0.127491, & -1.020980, & -0.863914 \\ \text { O, } & 0.689260, & -1.562688, & 0.002248 \\ \text { N, } & -1.143538, & -1.734614, & -1.250491 \\ \text { H, } & -1.247027, & -2.638114, & -0.805580 \\ \text { C, } & -2.163364, & -1.352041, & -2.225920 \\ \text { H, } & -2.342659, & -2.213338, & -2.869061 \\ \text { H, } & -1.777277, & -0.530278, & -2.817055 \\ \text { C, } & -3.451448, & -0.933182, & -1.534000 \\ \text { H, } & -3.231833, & -0.071090, & -0.902434 \\ \text { H, } & -3.830547, & -1.751502, & -0.911767 \\ \text { O, } & -4.371386, & -0.609554, & -2.575411 \\ \text { H, } & -5.177745, & -0.272270, & -2.174057\end{array}$




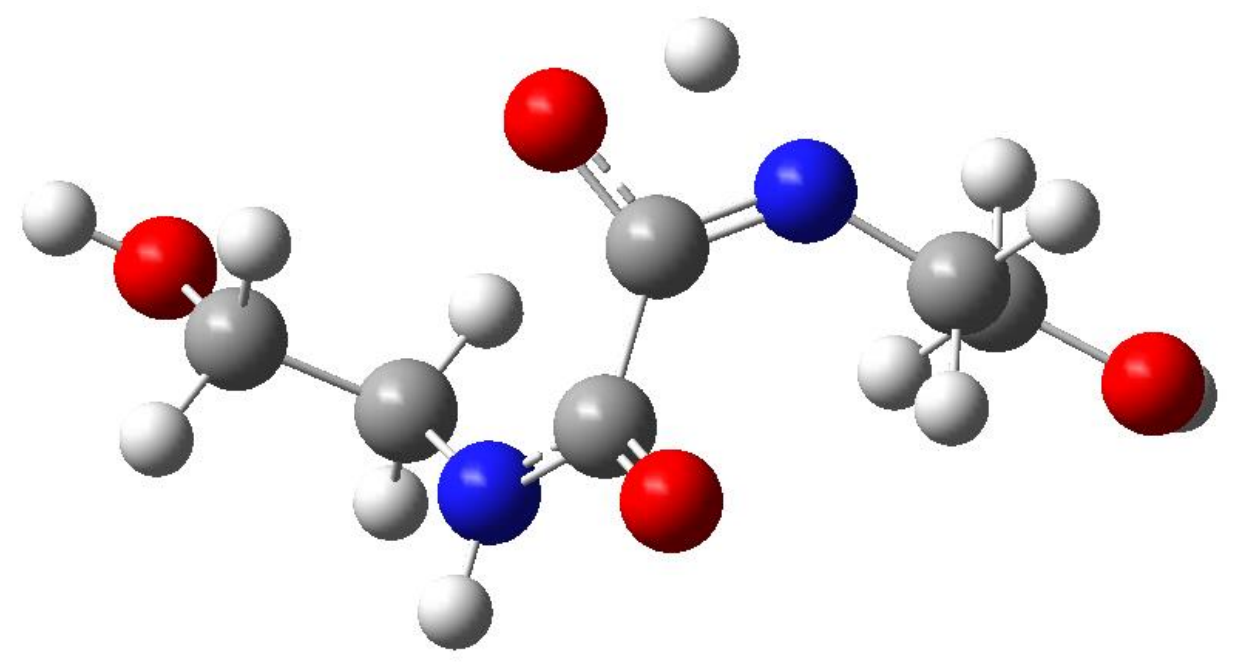

Formula:

Charge:

Energy:

Gibbs:

Multiplicity:

Dipole:

Setup:

Formula:

Charge:

Energy:

Gibbs:

Multiplicity:

Dipole:

Setup:

Formula:

Charge:

Energy:

Gibbs:

Multiplicity:

Dipole:
C6H12N2O4

0

-646.273915 a.u

-646.33166 a.u

1

5.1016

B3LYP, def2TZVP

C6H12N2O4

0

-646.295851 a.u

-646.352772 a.u

1

5.6037

M06, def2TZVP

C6H12N2O4

0

-645.887813 a.u

-645.944367 a.u

1

5.7101

$\begin{array}{lrlr}\text { O, } & 1.447734, & 0.472073, & 1.588200 \\ \text { C, } & 0.343855, & 2.494390, & -1.078072 \\ \text { H, } & 0.592797, & 3.553107, & -1.000260 \\ \text { H, } & 0.896511, & 2.081671, & -1.923827 \\ \text { C, } & -1.153987, & 2.352385, & -1.319198 \\ \text { H, } & -1.702341, & 2.734380, & -0.451881 \\ \text { H, } & -1.403555, & 1.292464, & -1.438431 \\ \text { O, } & -1.458973, & 3.094348, & -2.496988 \\ \text { H, } & -2.403761, & 3.026957, & -2.665058 \\ \text { H, } & 0.878965, & 1.651022, & 1.492782\end{array}$




$\begin{array}{lrrr}\text { N, } & 0.737858, & 1.834610, & 0.149707 \\ \text { C, } & 1.292247, & 0.678781, & 0.315833 \\ \text { C, } & 1.781256, & -0.278706, & -0.746878 \\ \text { O, } & 2.645848, & 0.104084, & -1.527008 \\ \text { N, } & 1.217548, & -1.495675, & -0.766633 \\ \text { H, } & 1.580231, & -2.119279, & -1.473608 \\ \text { C, } & 0.189775, & -2.006010, & 0.131258 \\ \text { H, } & -0.492079, & -2.627119, & -0.448095 \\ \text { H, } & -0.388502, & -1.177101, & 0.534251 \\ \text { C, } & 0.782827, & -2.818138, & 1.274228 \\ \text { H, } & 1.377092, & -3.644356, & 0.869059 \\ \text { H, } & 1.440084, & -2.174506, & 1.865012 \\ \text { O, } & -0.315776, & -3.297029, & 2.045304 \\ \text { H, } & 0.028386, & -3.794588, & 2.793119\end{array}$




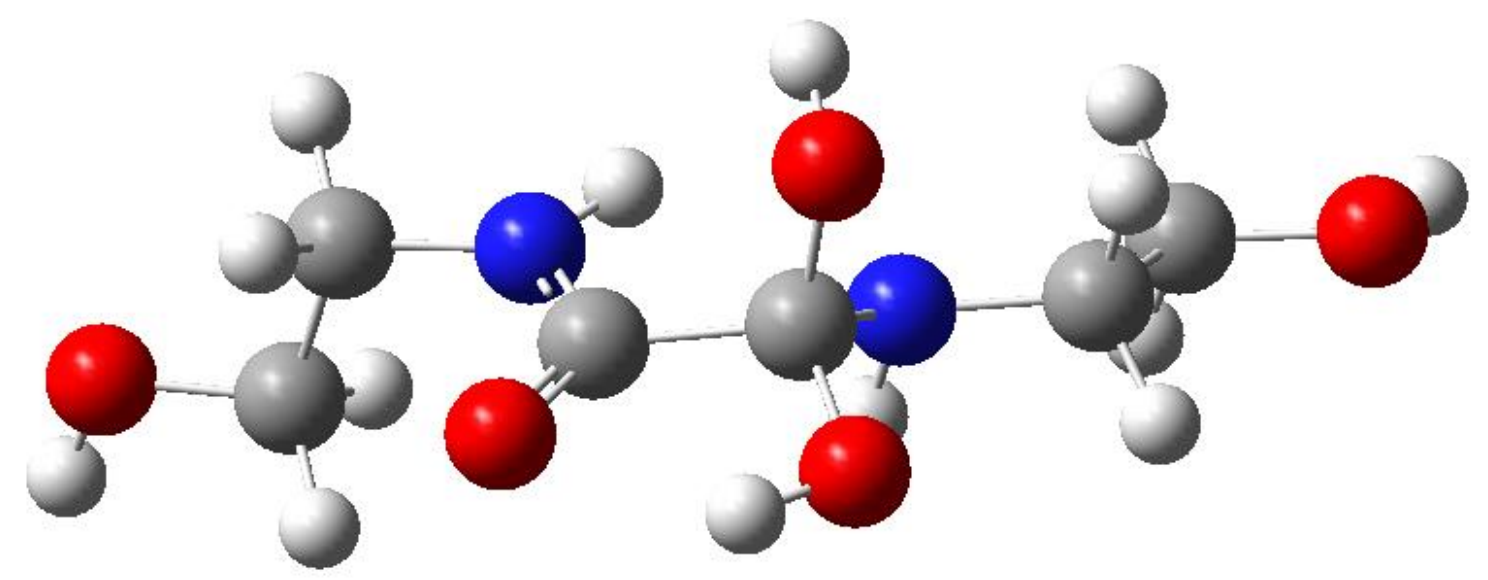

Formula:

Charge:

Energy:

Gibbs:

Multiplicity

Dipole: $\quad 8.1729$

$\mathrm{C} 6 \mathrm{H} 14 \mathrm{~N} 2 \mathrm{O} 5$

0

$-722.77291 \quad$ a.u

$-722.832829 \quad$ a.u

Setup:

Formula:

B3LYP, def2TZVP

Charge:

C6H14N2O5

Energy:

0

Gibbs:

-722.796982 a.u

Multiplicity:

Dipole:

-722.856868 a.u

1

8.4363

Setup:

Formula:

M06, def2TZVP

Charge:

C6H14N2O5

Energy:

0

Gibbs:

-722.351318 a.u

-722.410463 a.u

Multiplicity:

1

Dipole:

8.2788

$\begin{array}{lrlr}\text { O, } & 1.400448, & 0.975047, & 0.413879 \\ \text { C, } & -0.043957, & 2.741364, & -1.382230 \\ \text { H, } & 0.878117, & 3.039049, & -0.887097 \\ \text { H, } & 0.191572, & 2.546137, & -2.433078 \\ \text { C, } & -1.049939, & 3.874987, & -1.293119 \\ \text { H, } & -1.230601, & 4.120916, & -0.241773 \\ \text { H, } & -2.004273, & 3.562533, & -1.733497 \\ \text { O, } & -0.501782, & 4.985923, & -2.001504 \\ \text { H, } & -1.129158, & 5.713778, & -1.958920 \\ \text { H, } & -1.358576, & 1.177386, & -1.221437\end{array}$




$\begin{array}{lrrr}\text { H, } & 0.985470, & 1.424038, & 1.160822 \\ \text { N, } & -0.575247, & 1.556157, & -0.701219 \\ \text { C, } & 0.372405, & 0.506038, & -0.449813 \\ \text { O, } & 1.000575, & 0.043457, & -1.603954 \\ \text { H, } & 1.143972, & -0.907086, & -1.461507 \\ \text { C, } & -0.315745, & -0.707952, & 0.244839 \\ \text { O, } & 0.093681, & -1.842588, & 0.008920 \\ \text { N, } & -1.280025, & -0.403445, & 1.120744 \\ \text { H, } & -1.573972, & 0.560620, & 1.161552 \\ \text { C, } & -1.990541, & -1.403317, & 1.899965 \\ \text { H, } & -2.302475, & -0.954110, & 2.841470 \\ \text { H, } & -1.307296, & -2.221889, & 2.118792 \\ \text { C, } & -3.205713, & -1.941286, & 1.155048 \\ \text { H, } & -2.881187, & -2.389651, & 0.210809 \\ \text { H, } & -3.890629, & -1.118280, & 0.924941 \\ \text { O, } & -3.818809, & -2.905311, & 2.006504 \\ \text { H, } & -4.589937, & -3.264736, & 1.557495\end{array}$




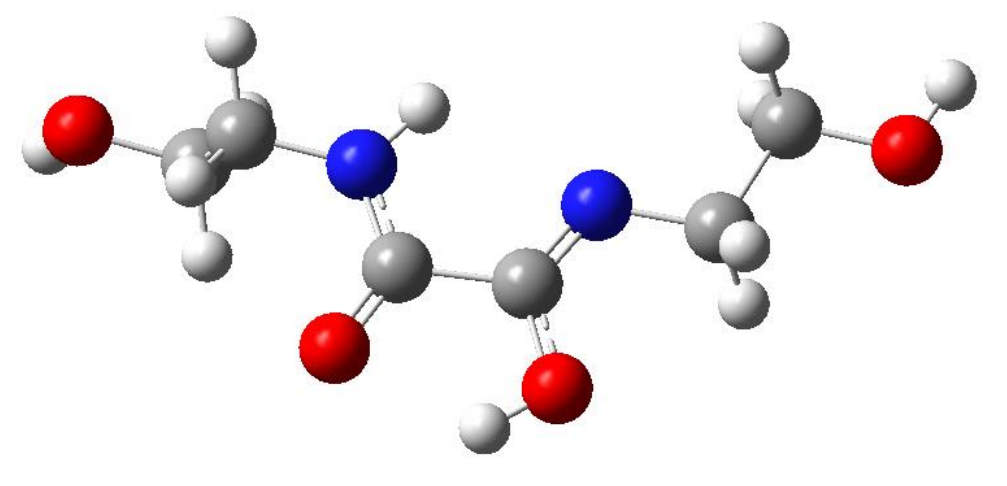

\begin{tabular}{|c|c|c|}
\hline Formula: & $\mathrm{C} 6 \mathrm{H} 12 \mathrm{~N} 2 \mathrm{O} 4$ & \\
\hline Charge: & 0 & \\
\hline Energy: & -646.333819 & a.u \\
\hline Gibbs: & -646.391647 & a.u \\
\hline Multiplicity: & 1 & \\
\hline Dipole: & 4.5846 & \\
\hline Setup: & \multicolumn{2}{|c|}{ B3LYP, def2TZVP } \\
\hline Formula: & \multicolumn{2}{|c|}{$\mathrm{C} 6 \mathrm{H} 12 \mathrm{~N} 2 \mathrm{O} 4$} \\
\hline Charge: & \multicolumn{2}{|l|}{0} \\
\hline Energy: & -646.355505 & a.u \\
\hline Gibbs: & -646.412901 & a.u \\
\hline Multiplicity: & \multicolumn{2}{|l|}{ 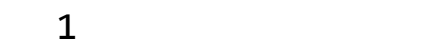 } \\
\hline Dipole: & \multicolumn{2}{|l|}{4.7760} \\
\hline Setup: & \multicolumn{2}{|l|}{ M06, def2TZVP } \\
\hline Formula: & \multicolumn{2}{|l|}{$\mathrm{C} 6 \mathrm{H} 12 \mathrm{~N} 2 \mathrm{O} 4$} \\
\hline Charge: & \multicolumn{2}{|l|}{0} \\
\hline Energy: & -645.947773 & a.u \\
\hline Gibbs: & -646.004045 & a.u \\
\hline Multiplicity: & 1 & \\
\hline Dipole: & 4.4338 & \\
\hline
\end{tabular}

$\begin{array}{rrrr}\text { C, } & -0.494948, & 2.777634, & -1.253572 \\ \text { H, } & 0.566836, & 3.028921, & -1.343606 \\ \text { H, } & -0.949790, & 2.987851, & -2.226966 \\ \text { C, } & -1.134891, & 3.655910, & -0.194542 \\ \text { H, } & -0.669446, & 3.455961, & 0.776527 \\ \text { H, } & -2.199878, & 3.413379, & -0.113299 \\ \text { O, } & -0.940850, & 5.012056, & -0.593288 \\ \text { H, } & -1.342761, & 5.584739, & 0.066566 \\ \text { N, } & -0.662116, & 1.376803, & -0.897194 \\ \text { C, } & -0.212184, & 0.496476, & -1.680730 \\ \text { O, } & 0.422691, & 0.734772, & -2.840911 \\ \text { H, } & 0.637051, & -0.143873, & -3.210568\end{array}$




$\begin{array}{lllr}\text { C, } & -0.351623, & -0.986582, & -1.385529 \\ \text { O, } & 0.098780, & -1.791887, & -2.203208 \\ \text { N, } & -0.955854, & -1.292706, & -0.236542 \\ \text { H, } & -1.289352, & -0.514455, & 0.312584 \\ \text { C, } & -1.199302, & -2.655830, & 0.197799 \\ \text { H, } & -0.404520, & -3.290763, & -0.189930 \\ \text { H, } & -1.164878, & -2.687372, & 1.285592 \\ \text { C, } & -2.546325, & -3.174172, & -0.291984 \\ \text { H, } & -3.348702, & -2.533767, & 0.089263 \\ \text { H, } & -2.571797, & -3.138648, & -1.385696 \\ \text { O, } & -2.672600, & -4.509049, & 0.189378 \\ \text { H, } & -3.513492, & -4.865719, & -0.112514\end{array}$




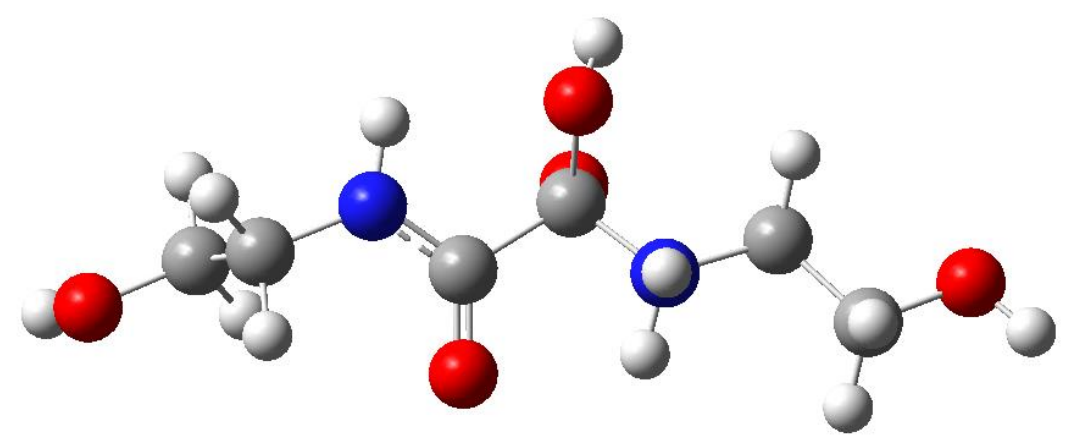

Formula:

Charge:

C6H14N2O5

Energy:

0

Gibbs:

$-722.755949$

a.u

Multiplicity:

$-722.814429$

a.u

Dipole:

5.6030

Setup:

Formula:

Charge:

Energy:

Gibbs:

B3LYP, def2TZVP

C6H14N2O5

0

-722.808110 a.u

-722.875038 a.u

Multiplicity: $\quad 1$

Dipole:

6.0120

Setup:

Formula:

Charge:

M06, def2TZVP

Energy:

C6H14N2O5

0

Gibbs:

-722.360119 a.u

Multiplicity:

-722.425744 a.u

Dipole:

1

7.1103

$\begin{array}{lr}\text { O, } & 0.654812, \\ \text { C, } & 0.118452, \\ \text { H, } & 1.122267, \\ \text { H, } & -0.450284, \\ \text { C, } & 0.138009, \\ \text { H, } & 0.689581, \\ \text { H, } & -0.881844, \\ \text { O, } & 0.776346, \\ \text { H, } & 0.797834, \\ \text { H, } & -1.484146, \\ \text { H, } & -0.049825, \\ \text { N, } & -0.521005, \\ \text { C, } & -0.497630, \\ \text { O, } & -1.580443,\end{array}$

-0.115252 ,

$-0.700935$

2.516074,

$-1.434844$

2.117401,

$-1.528480$

2.263067,

$-2.325645$

4.017529,

$-1.213027$

4.253540,

$-0.296234$

4.401678,

$-1.103155$

4.568122,

$-2.357851$

5.525657,

$-2.265727$

2.138888,

$-0.182693$

2.046169,

0.596643

1.816003,

$-0.284775$

0.348782 ,

$-0.372051$

0.037487 ,

$-1.298766$ 


$\begin{array}{llll}\text { H, } & -1.123512, & -0.330183, & -2.065387 \\ \text { C, } & -0.928348, & -0.123673, & 1.057407 \\ \text { O, } & -0.561880, & 0.513583, & 2.049049 \\ \text { N, } & -1.591557, & -1.284947, & 1.099313 \\ \text { H, } & -1.865289, & -1.681060, & 0.214980 \\ \text { C, } & -1.872717, & -2.003281, & 2.328701 \\ \text { H, } & -2.797217, & -2.565366, & 2.204641 \\ \text { H, } & -2.016305, & -1.280365, & 3.130196 \\ \text { C, } & -0.738236, & -2.949693, & 2.700047 \\ \text { H, } & -0.587844, & -3.677818, & 1.895935 \\ \text { H, } & 0.186639, & -2.375853, & 2.816831 \\ \text { O, } & -1.110509, & -3.590520, & 3.918047 \\ \text { H, } & -0.406981, & -4.194730, & 4.173763\end{array}$




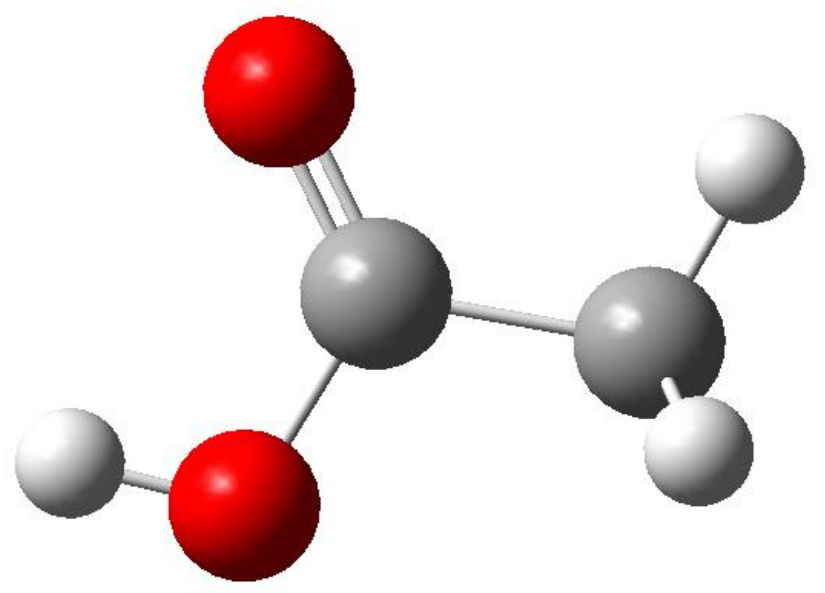

\begin{tabular}{|c|c|c|c|}
\hline \multirow{2}{*}{$\begin{array}{l}\text { Formula: } \\
\text { Charge: }\end{array}$} & \multicolumn{3}{|l|}{$\mathrm{C} 2 \mathrm{H} 4 \mathrm{O} 2$} \\
\hline & 0 & & \\
\hline Energy: & -229.125201 & a. & \\
\hline Gibbs: & -229.156867 & a. & \\
\hline Multiplicity: & 1 & & \\
\hline Dipole: & 2.1883 & & \\
\hline Setup: & \multicolumn{2}{|c|}{ B3LYP, def2TZVP } & \\
\hline Formula: & \multicolumn{2}{|c|}{$\mathrm{C} 2 \mathrm{H} 4 \mathrm{O} 2$} & \\
\hline Charge: & \multicolumn{2}{|l|}{0} & \\
\hline Energy: & -229.133815 & $\mathrm{a} \cdot \mathrm{u}$ & \\
\hline Gibbs: & \multicolumn{2}{|c|}{-229.165553 a.u } & \\
\hline \multirow{2}{*}{$\begin{array}{l}\text { Multiplicity: } \\
\text { Dipole: }\end{array}$} & \multicolumn{2}{|l|}{1} & \\
\hline & \multicolumn{2}{|l|}{2.2391} & \\
\hline \multirow{2}{*}{$\begin{array}{l}\text { Setup: } \\
\text { Formula: }\end{array}$} & \multicolumn{2}{|l|}{ M06, def2TZVP } & \\
\hline & \multicolumn{2}{|l|}{$\mathrm{C} 2 \mathrm{H} 4 \mathrm{O} 2$} & \\
\hline Charge: & \multicolumn{2}{|l|}{0} & \\
\hline Energy: & -228.997699 & a.u & \\
\hline \multirow{3}{*}{$\begin{array}{l}\text { Gibbs: } \\
\text { Multiplicity: } \\
\text { Dipole: }\end{array}$} & \multirow{2}{*}{\multicolumn{2}{|c|}{$\begin{array}{ll}-229.029478 & a . u\end{array}$}} & \\
\hline & & & \\
\hline & \multicolumn{2}{|l|}{2.2083} & \\
\hline 0.645 & 0.600 & 592, & -0.127886 \\
\hline 1.852 & 0.657 & 174, & -0.127975 \\
\hline-0.132 & 1.703 & 363, & -0.127848 \\
\hline 0.446 & 2.480 & 920 , & -0.127875 \\
\hline-0.175 & -0.655 & 235, & -0.127846 \\
\hline$-0.81 c$ & -0.672 & 046 & 0.750806 \\
\hline-0.826 & -0.671 & 913 , & -1.006247 \\
\hline $0.47 \epsilon$ & -1.522 & 471, & -0.128028 \\
\hline
\end{tabular}




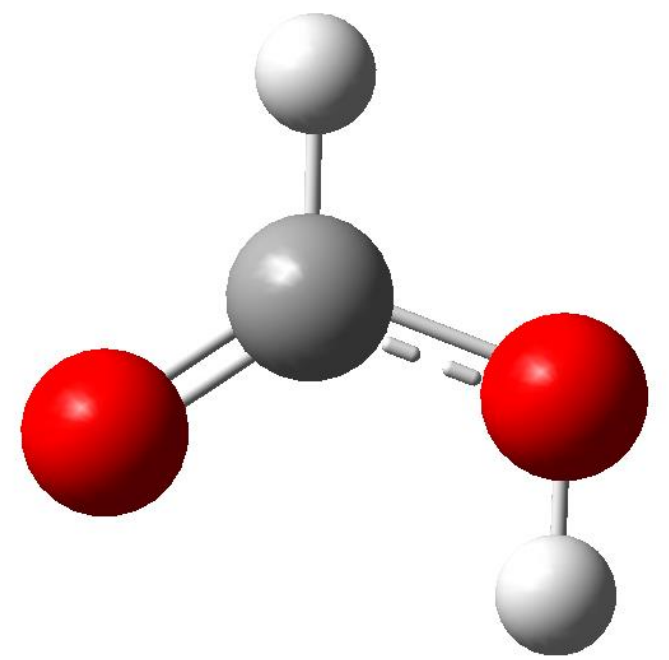

$\begin{array}{lll}\text { Formula: } & \mathrm{CH} 2 \mathrm{O} 2 & \\ \text { Charge: } & 0 & \\ \text { Energy: } & -189.811772 & \text { a.u } \\ \text { Gibbs: } & -189.839023 & \text { a.u }\end{array}$

Multiplicity: $\quad 1$

Dipole:

1.9528

Setup:

Formula:

B3LYP, def2TZVP

Charge:

$\mathrm{CH} 2 \mathrm{O} 2$

Energy:

0

Gibbs:

-189.820151 a.u

Multiplicity:

-189.847404 a.u

Dipole:

1

1.9868

Setup:

Formula:

Charge:

M06, def2TZVP

$\mathrm{CH} 2 \mathrm{O} 2$

0

Energy:

-189.719181 a.u

Gibbs:

-189.746414 a.u

Multiplicity:

1

Dipole:

1.9664

$\begin{array}{lrrr}\text { C, } & 0.645457, & 0.639335, & -0.127881 \\ \text { O, } & 1.848173, & 0.627255, & -0.127895 \\ \text { H, } & 0.010378, & -0.253546, & -0.127855 \\ \text { O, } & -0.115939, & 1.739345, & -0.127894 \\ \text { H, } & 0.461001, & 2.521044, & -0.127919\end{array}$



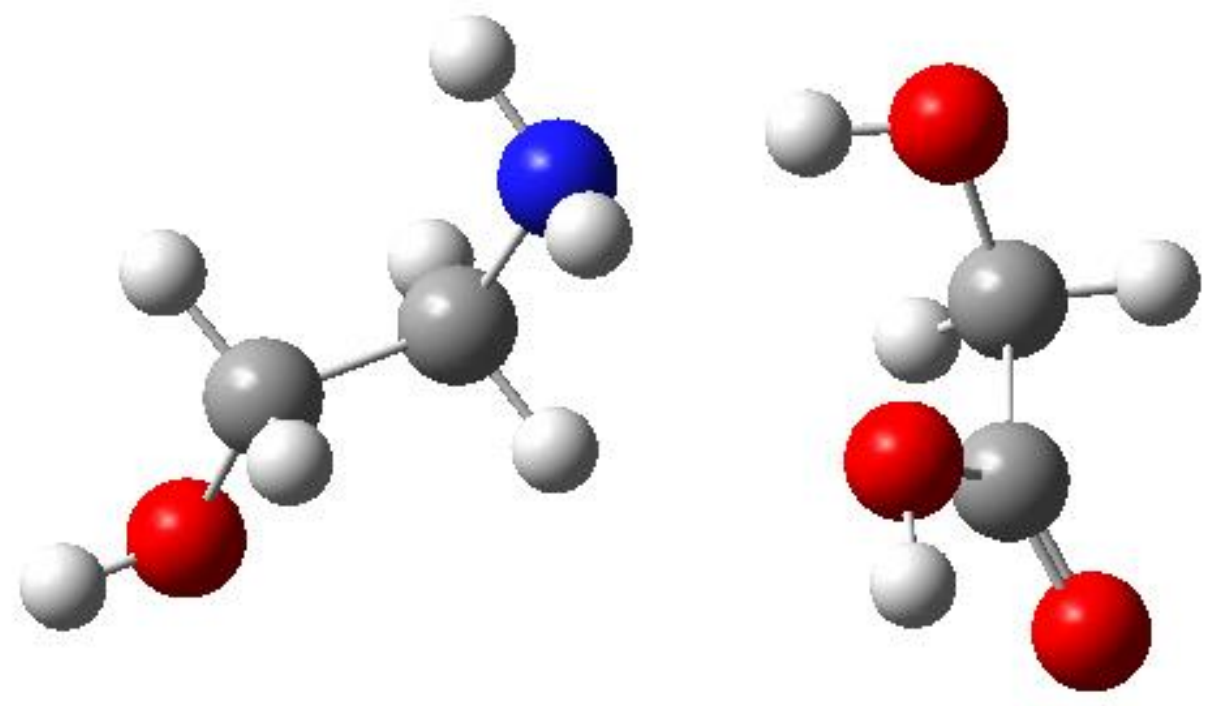

Formula:

Charge:

Energy:

Gibbs:

Multiplicity:

Dipole:

Setup:

Formula:

Charge:

Energy:

Gibbs:

Multiplicity:

Dipole:

Setup:

Formula:

Charge:

Energy:

Gibbs:

Multiplicity:

Dipole:
C4H11NO4

0

-514.745924 a.u

$-514.799134 \quad$ a.u

1

5.7895

B3LYP, def2TZVP

C4H11NO4

0

-439.523561 a.u

-439.576776 a.u

1

4.7221

M06, def2TZVP

$\mathrm{C} 4 \mathrm{H} 11 \mathrm{NO} 4$

0

-439.238687 a.u

-439.292853 a.u

1

4.3740 $\begin{array}{ll}\text { O, } & -1.986764, \\ \text { C, } & -6.295042, \\ H, & -6.388489, \\ H, & -5.618406, \\ \text { C, } & -7.658442, \\ H, & -8.353542, \\ H, & -7.566519, \\ \text { O, } & -8.106338,\end{array}$

1.981519,

0.022867 ,

-0.199948 ,

0.873095 ,

0.419654 ,

-0.424300 ,

0.654368 ,

1.548728,
$-0.912543$

$-0.088238$

0.975321

$-0.179741$

$-0.636851$

$-0.539301$

$-1.705331$

0.105166 


$\begin{array}{llrr}\text { H, } & -8.967458, & 1.819706, & -0.224888 \\ \text { H, } & -5.552405, & -0.939319, & -1.746309 \\ \text { H, } & -6.273778, & -1.950818, & -0.681108 \\ \text { N, } & -5.686375, & -1.128821, & -0.759443 \\ \text { C, } & -2.572594, & 0.928477, & -0.950541 \\ \text { O, } & -3.383504, & 0.581908, & -1.976494 \\ \text { H, } & -3.360425, & 1.315854, & -2.609296 \\ \text { C, } & -2.511772, & -0.108997, & 0.161855 \\ \text { H, } & -3.050990, & 0.344152, & 1.006830 \\ \text { H, } & -1.464834, & -0.167493, & 0.461021 \\ \text { O, } & -2.963764, & -1.392981, & -0.150565 \\ \text { H, } & -3.935398, & -1.360363, & -0.317110\end{array}$




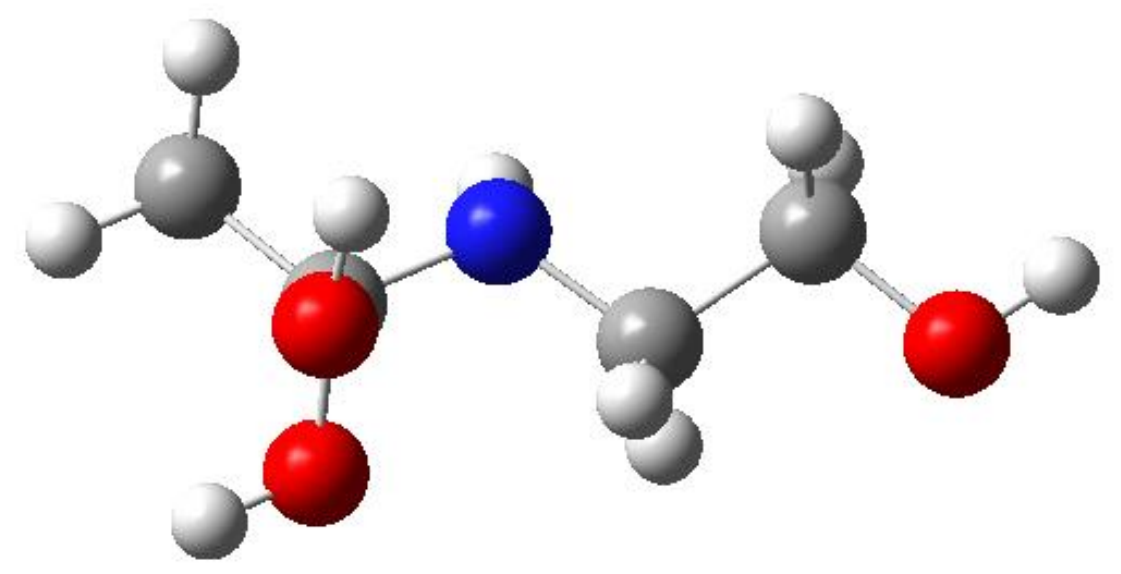

\begin{tabular}{|c|c|c|}
\hline Formula: & $\mathrm{C} 4 \mathrm{H} 11 \mathrm{NO} 3$ & \\
\hline Charge: & 0 & \\
\hline Energy: & -439.490784 & a.u \\
\hline Gibbs: & -439.536208 & a.u \\
\hline Multiplicity: & 1 & \\
\hline Dipole: & 3.7152 & \\
\hline Setup: & B3LYP, def2TZVP & \\
\hline Formula: & $\mathrm{C} 4 \mathrm{H} 11 \mathrm{NO} 3$ & \\
\hline Charge: & 0 & \\
\hline Energy: & -439.222077 & a.u \\
\hline Gibbs: & -439.267114 & a.u \\
\hline Multiplicity: & 1 & \\
\hline Dipole: & 3.8149 & \\
\hline $\begin{array}{l}\text { Setup: } \\
\text { Formula: }\end{array}$ & M06, def2TZVP & \\
\hline Charge: & 0 & \\
\hline Energy: & -439.504076 & a.u \\
\hline Gibbs: & -439.549519 & a.u \\
\hline Multiplicity: & 1 & \\
\hline Dipole: & 3.9234 & \\
\hline
\end{tabular}

$\begin{array}{lrlr}\text { O, } & 1.669241, & 1.350536, & 0.481057 \\ \text { C, } & -0.155191, & 2.506077, & -1.513040 \\ \text { H, } & 0.858007, & 2.872871, & -1.357477 \\ \text { H, } & -0.185907, & 2.037696, & -2.502201 \\ \text { C, } & -1.120787, & 3.677296, & -1.483729 \\ \text { H, } & -1.033562, & 4.198257, & -0.524690 \\ \text { H, } & -2.151445, & 3.313498, & -1.574254 \\ \text { O, } & -0.788159, & 4.536931, & -2.575035 \\ \text { H, } & -1.397435, & 5.281084, & -2.572462 \\ \text { H, } & -1.334542, & 1.077876, & -0.621278 \\ \text { H, } & 1.339089, & 1.891562, & 1.207783\end{array}$




$\begin{array}{lrrr}\text { N, } & -0.473486, & 1.574018, & -0.432131 \\ \text { C, } & 0.558599, & 0.645272, & -0.066453 \\ \text { O, } & 1.063113, & -0.002593, & -1.223708 \\ \text { H, } & 1.806114, & -0.557951, & -0.960237 \\ \text { C, } & 0.019810, & -0.374851, & 0.932259 \\ \text { H, } & -0.783609, & -0.958032, & 0.483420 \\ \text { H, } & 0.822714, & -1.050352, & 1.223320 \\ \text { H, } & -0.358480, & 0.126083, & 1.822245\end{array}$




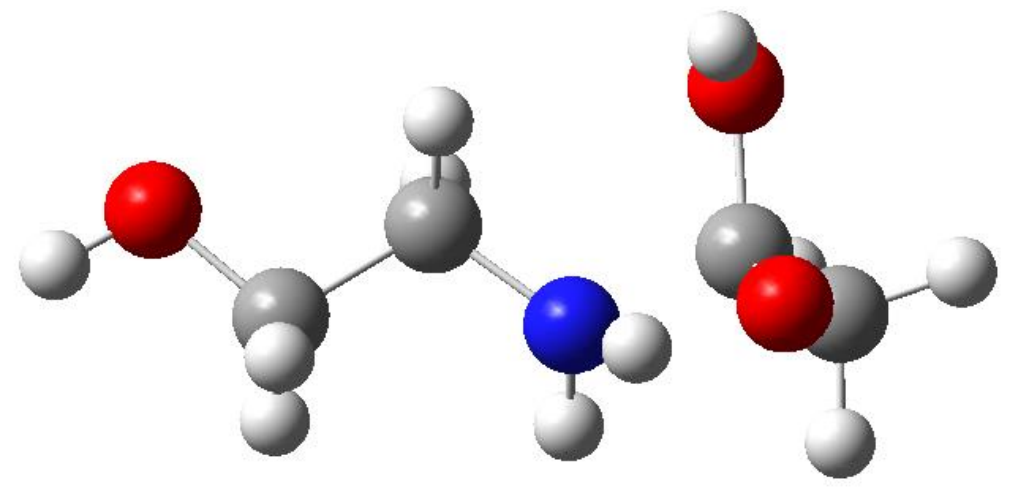

$\begin{array}{lll}\text { Formula: } & \text { C4H11NO3 } & \\ \text { Charge: } & 0 & \\ \text { Energy: } & -439.449503 & \text { a.u } \\ \text { Gibbs: } & -439.494321 & \text { a.u }\end{array}$

Multiplicity: 1

Dipole: $\quad 5.6655$

Setup: $\quad$ B3LYP, def2TZVP

Formula: $\quad$ C4H11NO3

Charge: $\quad 0$

Energy: $\quad-439.463154$ a.u

Gibbs: $\quad-439.507829$ a.u

Multiplicity: $\quad 1$

Dipole: $\quad 5.8415$

Setup: $\quad$ M06, def2TZVP

Formula: $\quad \mathrm{C} 4 \mathrm{H} 11 \mathrm{NO3}$

Charge: $\quad 0$

Energy: $\quad-439.178034$ a.u

Gibbs: $\quad-439.222572$ a.u

Multiplicity: $\quad 1$

Dipole: $\quad 5.9283$

$\begin{array}{lrlr}\text { O, } & 0.895541, & 0.842915, & 1.285733 \\ \text { C, } & -0.153023, & 2.473850, & -1.479008 \\ \text { H, } & 0.880786, & 2.809847, & -1.489857 \\ \text { H, } & -0.324685, & 1.882805, & -2.380540 \\ \text { C, } & -1.077468, & 3.679322, & -1.464537 \\ \text { H, } & -0.898890, & 4.268906, & -0.559346 \\ \text { H, } & -2.122957, & 3.350698, & -1.453188 \\ \text { O, } & -0.789805, & 4.431296, & -2.640165 \\ \text { H, } & -1.363059, & 5.203772, & -2.655878 \\ \text { H, } & -1.333435, & 1.355802, & -0.211681 \\ \text { H, } & 0.152111, & 1.822420, & 0.847174 \\ \text { N, } & -0.363817, & 1.650205, & -0.284226 \\ \text { C, } & 0.593129, & 0.434857, & 0.024084 \\ \text { O, } & 1.685459, & 0.444536, & -0.859157 \\ \text { H, } & 2.335268, & 1.043591, & -0.470133\end{array}$




$\begin{array}{lrrr}\text { C, } & -0.123067, & -0.893087, & -0.126063 \\ \text { H, } & 0.539850, & -1.682233, & 0.224652 \\ \text { H, } & -1.027416, & -0.900898, & 0.479980 \\ \text { H, } & -0.380310, & -1.085304, & -1.167152\end{array}$



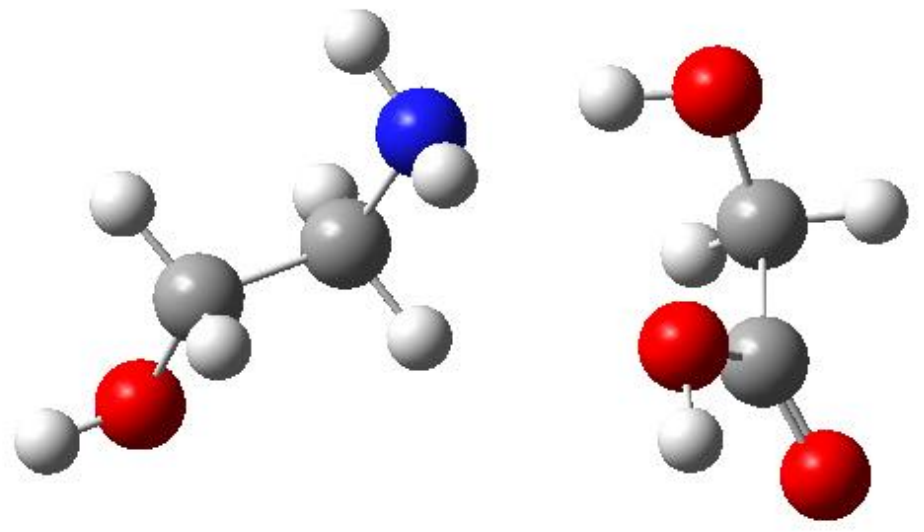

Formula:

Charge:

Energy:

Gibbs:

Multiplicity:

Dipole:
C4H11NO4

0

$-514.745924$

$-514.799134$

1

5.7895 a.u

a.u

$\begin{array}{llrr}\text { O, } & -1.986764, & 1.981519, & -0.912543 \\ \text { C, } & -6.295042, & 0.022867, & -0.088238 \\ \text { H, } & -6.388489, & -0.199948, & 0.975321 \\ \text { H, } & -5.618406, & 0.873095, & -0.179741 \\ \text { C, } & -7.658442, & 0.419654, & -0.636851 \\ \text { H, } & -8.353542, & -0.424300, & -0.539301 \\ \text { H, } & -7.566519, & 0.654368, & -1.705331 \\ \text { O, } & -8.106338, & 1.548728, & 0.105166 \\ \text { H, } & -8.967458, & 1.819706, & -0.224888 \\ \text { H, } & -5.552405, & -0.939319, & -1.746309 \\ \text { H, } & -6.273778, & -1.950818, & -0.681108 \\ \text { N, } & -5.686375, & -1.128821, & -0.759443 \\ \text { C, } & -2.572594, & 0.928477, & -0.950541 \\ \text { O, } & -3.383504, & 0.581908, & -1.976494 \\ \text { H, } & -3.360425, & 1.315854, & -2.609296 \\ \text { C, } & -2.511772, & -0.108997, & 0.161855 \\ \text { H, } & -3.050990, & 0.344152, & 1.006830 \\ \text { H, } & -1.464834, & -0.167493, & 0.461021 \\ \text { O, } & -2.963764, & -1.392981, & -0.150565 \\ \text { H, } & -3.935398, & -1.360363, & -0.317110\end{array}$




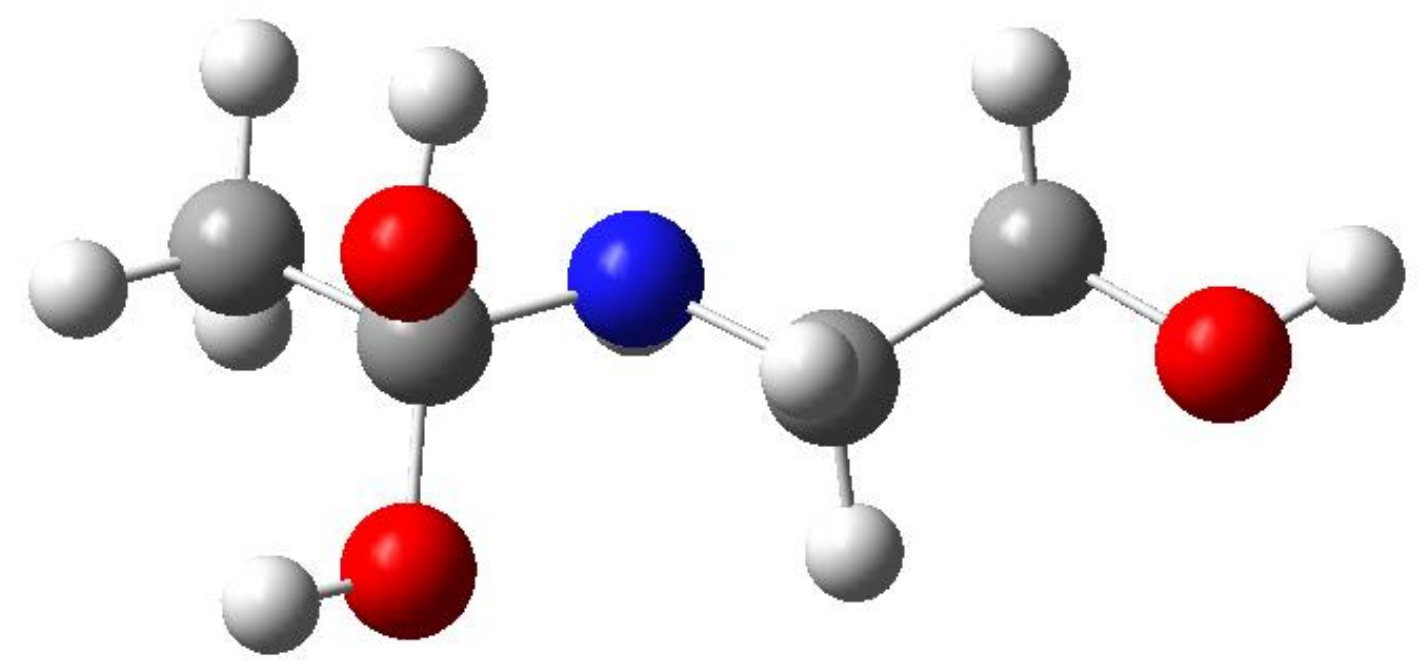

\begin{tabular}{|c|c|c|}
\hline Formula: & $\mathrm{C} 4 \mathrm{H} 11 \mathrm{NO} 3$ & \\
\hline Charge: & 0 & \\
\hline Energy: & -439.490784 & a.u \\
\hline Gibbs: & -439.536208 & a.u \\
\hline Multiplicity: & 1 & \\
\hline Dipole: & 3.7152 & \\
\hline Setup: & B3LYP, def2TZVP & \\
\hline Formula: & $\mathrm{C} 4 \mathrm{H} 11 \mathrm{NO} 03$ & \\
\hline Charge: & 0 & \\
\hline Energy: & -439.222077 & a.u \\
\hline Gibbs: & -439.267114 & a.u \\
\hline Multiplicity: & 1 & \\
\hline Dipole: & 3.8149 & \\
\hline $\begin{array}{l}\text { Setup: } \\
\text { Formula: }\end{array}$ & M06, def2TZVP & \\
\hline Charge: & $\theta$ & \\
\hline Energy: & -439.504076 & a.u \\
\hline Gibbs: & -439.549519 & a.u \\
\hline Multiplicity: & 1 & \\
\hline Dipole: & 3.9234 & \\
\hline
\end{tabular}

$\begin{array}{lrlr}\text { O, } & 1.669241, & 1.350536, & 0.481057 \\ \text { C, } & -0.155191, & 2.506077, & -1.513040 \\ \text { H, } & 0.858007, & 2.872871, & -1.357477 \\ \text { H, } & -0.185907, & 2.037696, & -2.502201 \\ \text { C, } & -1.120787, & 3.677296, & -1.483729 \\ \text { H, } & -1.033562, & 4.198257, & -0.524690 \\ \text { H, } & -2.151445, & 3.313498, & -1.574254 \\ \text { O, } & -0.788159, & 4.536931, & -2.575035 \\ \text { H, } & -1.397435, & 5.281084, & -2.572462 \\ \text { H, } & -1.334542, & 1.077876, & -0.621278 \\ \text { H, } & 1.339089, & 1.891562, & 1.207783\end{array}$




$\begin{array}{lrrr}\text { N, } & -0.473486, & 1.574018, & -0.432131 \\ \text { C, } & 0.558599, & 0.645272, & -0.066453 \\ \text { O, } & 1.063113, & -0.002593, & -1.223708 \\ \text { H, } & 1.806114, & -0.557951, & -0.960237 \\ \text { C, } & 0.019810, & -0.374851, & 0.932259 \\ \text { H, } & -0.783609, & -0.958032, & 0.483420 \\ \text { H, } & 0.822714, & -1.050352, & 1.223320 \\ \text { H, } & -0.358480, & 0.126083, & 1.822245\end{array}$



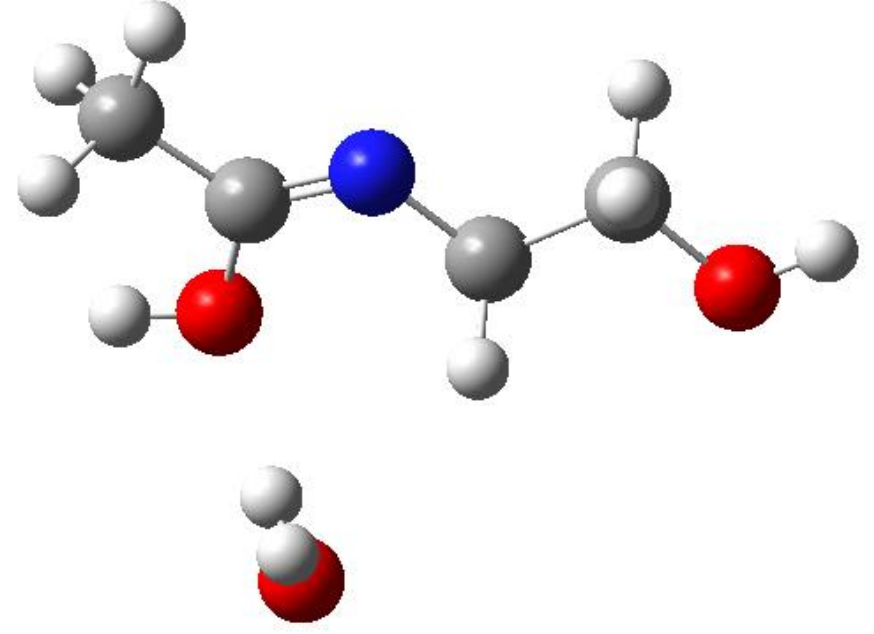

Formula:

C4H11NO3

Charge:

0

Energy:

$-439.49509$

Gibbs:

$-439.54597$

a.u

Multiplicity:

1

Dipole:

5.1040

$\begin{array}{lrrr}\text { O, } & 2.756872, & 1.416750, & 0.067987 \\ \text { C, } & -0.387843, & 2.688093, & -1.030404 \\ \text { H, } & 0.510578, & 2.678581, & -0.406662 \\ \text { H, } & -0.083405, & 3.061353, & -2.013609 \\ \text { C, } & -1.403938, & 3.638842, & -0.425322 \\ \text { H, } & -1.700074, & 3.275300, & 0.564628 \\ \text { H, } & -2.300718, & 3.668009, & -1.053717 \\ \text { O, } & -0.789950, & 4.925604, & -0.341235 \\ \text { H, } & -1.421698, & 5.539897, & 0.043959 \\ \text { H, } & 2.239854, & 1.172957, & -0.712782 \\ \text { H, } & 2.326702, & 0.946294, & 0.789066 \\ \text { N, } & -0.971449, & 1.355915, & -1.129988 \\ \text { C, } & -0.305555, & 0.432377, & -1.670644 \\ \text { O, } & 0.965893, & 0.659066, & -2.149641 \\ \text { H, } & 1.313711, & -0.133337, & -2.576427 \\ \text { C, } & -0.815222, & -0.965064, & -1.832722 \\ \text { H, } & -0.818489, & -1.248834, & -2.887244 \\ \text { H, } & -0.168272, & -1.667536, & -1.303391 \\ \text { H, } & -1.823986, & -1.041116, & -1.439661\end{array}$





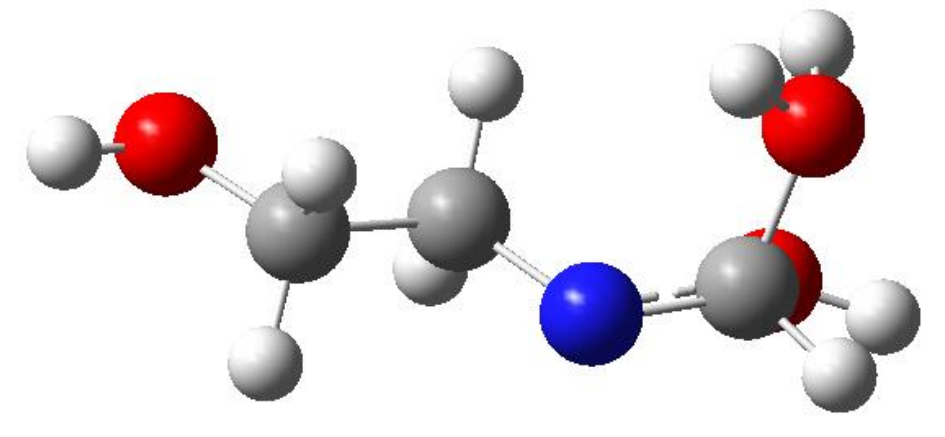

Formula:

Charge:

C4H11NO3

Energy:

0

Gibbs:

$-439.384574$

a.u

$-439.428837$

a.u

Multiplicity: 1

Dipole:

7.2820

Setup:

Formula:

Charge:

B3LYP, def2TZVP

$\mathrm{C} 4 \mathrm{H} 11 \mathrm{NO} 3$

0

Energy:

-439.397906 a.u

Gibbs:

-439.442137 a.u

Multiplicity: $\quad 1$

Dipole:

7.4331

Setup:

Formula:

M06, def2TZVP

Charge:

$\mathrm{C} 4 \mathrm{H} 11 \mathrm{NO} 3$

Energy:

0

Gibbs:

-439.119286 a.u

Multiplicity: $\quad 1$

Dipole:

$-439.162663 a . u$

1

7.4133

$\begin{array}{lrlr}\text { O, } & 1.291860, & 0.447134, & -0.394356 \\ \text { C, } & -0.326636, & 2.798691, & -1.096103 \\ \text { H, } & 0.664857, & 3.047899, & -0.673197 \\ \text { H, } & -0.290378, & 3.150538, & -2.139445 \\ \text { C, } & -1.347592, & 3.645940, & -0.358142 \\ \text { H, } & -1.357382, & 3.364057, & 0.700958 \\ \text { H, } & -2.344629, & 3.443657, & -0.765318 \\ \text { O, } & -1.001655, & 5.028120, & -0.516307 \\ \text { H, } & -1.658245, & 5.558091, & -0.055264 \\ \text { H, } & 0.968033, & 0.873789, & 0.423589 \\ \text { H, } & 2.123431, & 0.858660, & -0.696407 \\ \text { N, } & -0.703719, & 1.400429, & -0.985396 \\ \text { C, } & 0.240219, & 0.517731, & -1.360776\end{array}$




$\begin{array}{llll}\text { O, } & 1.029991, & 0.876946, & -2.542259 \\ \text { H, } & 1.356475, & 0.074713, & -2.964902 \\ \text { C, } & -0.279317, & -0.912527, & -1.497226 \\ \text { H, } & -0.970427, & -0.954607, & -2.334791 \\ \text { H, } & 0.535696, & -1.619449, & -1.663038 \\ \text { H, } & -0.807573, & -1.196659, & -0.589432\end{array}$




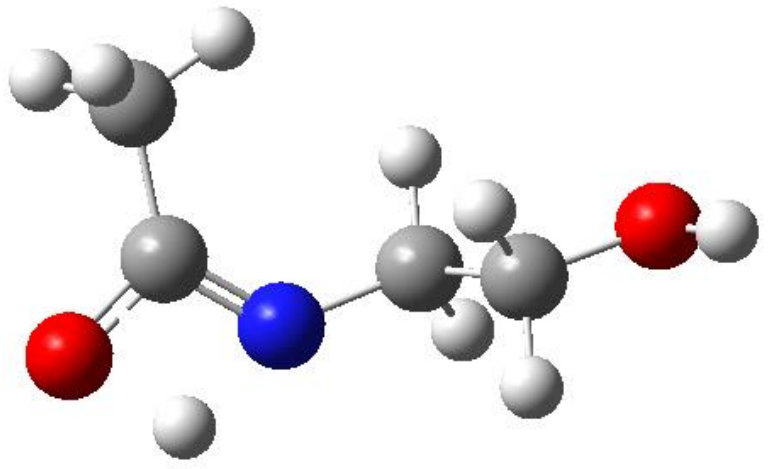

\begin{tabular}{|c|c|c|}
\hline Formula: & C4H9NO2 & \\
\hline Charge: & 0 & \\
\hline Energy: & -363.005697 & a.u \\
\hline Gibbs: & -363.048335 & a.u \\
\hline Multiplicity: & 1 & \\
\hline Dipole: & 3.3092 & \\
\hline Setup: & \multicolumn{2}{|c|}{ B3LYP, def2TZVP } \\
\hline Formula: & \multicolumn{2}{|l|}{ C4H9NO2 } \\
\hline Charge: & \multicolumn{2}{|l|}{0} \\
\hline Energy: & \multicolumn{2}{|c|}{-363.016822 a.u } \\
\hline Gibbs: & \multicolumn{2}{|c|}{-363.059511 a.u } \\
\hline Multiplicity: & \multicolumn{2}{|l|}{1} \\
\hline Dipole: & \multicolumn{2}{|l|}{3.3600} \\
\hline Setup: & \multicolumn{2}{|l|}{ M06, def2TZVP } \\
\hline Formula: & \multicolumn{2}{|l|}{ C4H9NO2 } \\
\hline Charge: & \multicolumn{2}{|l|}{0} \\
\hline Energy: & -362.773425 & a.u \\
\hline Gibbs: & -362.815620 & a.u \\
\hline Multiplicity: & 1 & \\
\hline Dipole: & 3.4322 & \\
\hline
\end{tabular}

$\begin{array}{lrrr}\text { O, } & 0.666933, & 0.570332, & 1.388275 \\ \text { C, } & 0.349299, & 2.745354, & -1.352239 \\ \text { H, } & 1.072943, & 3.561059, & -1.394182 \\ \text { H, } & 0.538789, & 2.097673, & -2.212055 \\ \text { C, } & -1.054639, & 3.327522, & -1.461513 \\ \text { H, } & -1.257302, & 3.956573, & -0.588381 \\ \text { H, } & -1.786803, & 2.512844, & -1.469261 \\ \text { O, } & -1.106086, & 4.083073, & -2.670106 \\ \text { H, } & -1.984134, & 4.466904, & -2.753761 \\ \text { H, } & 0.683949, & 1.862636, & 1.212578 \\ \text { N, } & 0.525450, & 2.022866, & -0.113393 \\ \text { C, } & 0.506414, & 0.739838, & 0.106439 \\ \text { C, } & 0.345952, & -0.398119, & -0.834099 \\ \text { H, } & 1.198522, & -1.069177, & -0.733946 \\ \text { H, } & -0.548504, & -0.959257, & -0.563700 \\ \text { H, } & 0.262711, & -0.067998, & -1.866071\end{array}$




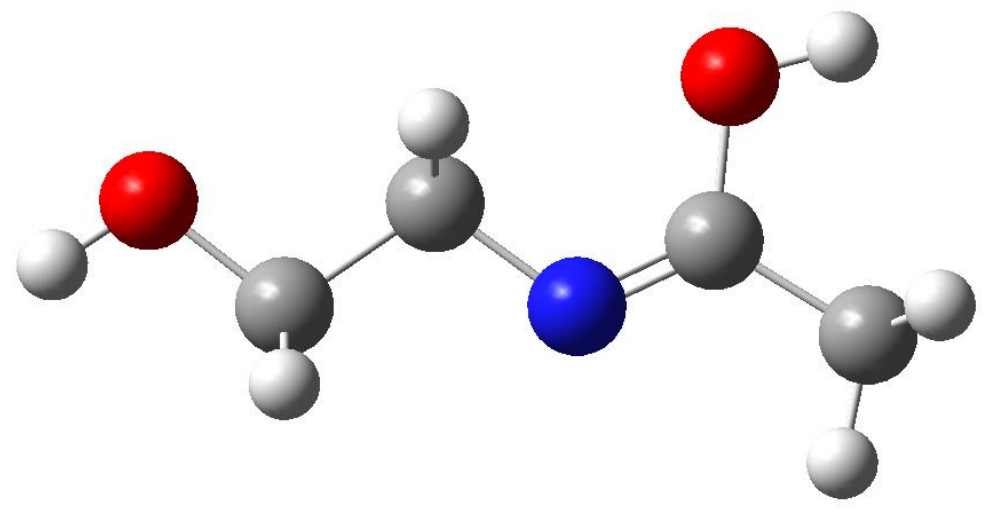

Formula:

Charge:

Energy:

Gibbs:

Multiplicity:

Dipole:

Setup:

Formula:

Charge:

Energy:

Gibbs:

Multiplicity:

Dipole:

Setup:

Formula:

Charge:

Energy:

Gibbs:

Multiplicity

Dipole:
$\mathrm{C} 4 \mathrm{H} 9 \mathrm{NO} 2$

0

$-363.047596 \quad$ a.u

-363.08962 a.u

1

3.3024

B3LYP, def2TZVP

C4H9NO2

0

-363.058448 a.u

-363.100466 a.u

1

3.3803

M06, def2TZVP

0

-362.814197 a.u

-362.855885 a.u

1

3.3630

$\begin{array}{lrrr}\text { C, } & -0.370337, & 2.777165, & -1.136285 \\ \text { H, } & 0.618779, & 2.963694, & -0.704779 \\ \text { H, } & -0.320824, & 3.103818, & -2.180402 \\ \text { C, } & -1.401157, & 3.613155, & -0.400546 \\ \text { H, } & -1.442454, & 3.302703, & 0.649190 \\ \text { H, } & -2.390604, & 3.443155, & -0.838961 \\ \text { O, } & -1.012180, & 4.982595, & -0.519169 \\ \text { H, } & -1.656051, & 5.523327, & -0.052425 \\ \text { N, } & -0.730944, & 1.368428, & -1.040308 \\ \text { C, } & 0.018934, & 0.518476, & -1.599631 \\ \text { O, } & 1.140235, & 0.903293, & -2.278321 \\ \text { H, } & 1.595622, & 0.135325, & -2.642111 \\ \text { C, } & -0.253951, & -0.953953, & -1.565894 \\ \text { H, } & -0.365251, & -1.342169, & -2.580509 \\ \text { H, } & 0.579579, & -1.483395, & -1.099601 \\ \text { H, } & -1.161955, & -1.153574, & -1.005641\end{array}$




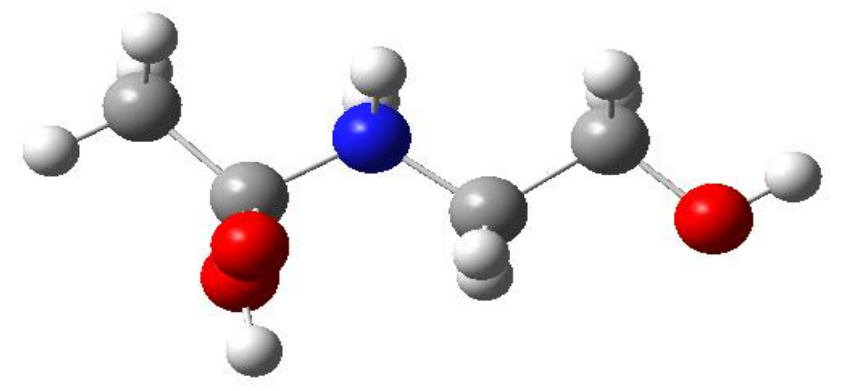

$\begin{array}{lll}\text { Formula: } & \text { C4H11NO3 } & \\ \text { Charge: } & 0 & \\ \text { Energy: } & -439.467264 & \text { a.u } \\ \text { Gibbs: } & -439.513833 & \text { a.u }\end{array}$

Multiplicity: 1

Dipole: $\quad 9.3164$

Setup:

Formula:

Charge:

Energy:

Gibbs:

B3LYP, def2TZVP

$\mathrm{C} 4 \mathrm{H} 11 \mathrm{NO} 3$

0

-400.175576 a.u

Multiplicity: $\quad 1$

Dipole:

-400.215979 a.u

1

Setup: $\quad$ M06, def2TZVP

Formula: $\quad$ C4H11NO3

Charge: $\quad 0$

Energy: $\quad-399.924642$ a.u

Gibbs: $\quad-399.964981$ a.u

Multiplicity: $\quad 1$

Dipole: $\quad 9.5491$

$\begin{array}{lllr}\text { O, } & 1.892688, & 0.972249, & 0.103652 \\ \text { C, } & 0.058416, & 2.483543, & -1.414190 \\ \text { H, } & 1.105117, & 2.757939, & -1.337649 \\ \text { H, } & -0.085149, & 1.848214, & -2.282318 \\ \text { C, } & -0.834807, & 3.708612, & -1.483793 \\ \text { H, } & -0.692871, & 4.329785, & -0.592072 \\ \text { H, } & -1.888412, & 3.411067, & -1.523896 \\ \text { O, } & -0.449024, & 4.403285, & -2.662999 \\ \text { H, } & -1.001221, & 5.186378, & -2.751390 \\ \text { H, } & -1.211366, & 1.314993, & -0.278290 \\ \text { H, } & -0.223408, & 2.267908, & 0.613637 \\ \text { N, } & -0.253557, & 1.660346, & -0.205852 \\ \text { C, } & 0.676364, & 0.550524, & 0.048002 \\ \text { O, } & 0.398456, & -0.358826, & -1.081975 \\ \text { H, } & 1.191500, & -0.277234, & -1.624146 \\ \text { C, } & 0.145583, & -0.186019, & 1.297421 \\ \text { H, } & -0.876500, & -0.549939, & 1.175661 \\ \text { H, } & 0.800335, & -1.036922, & 1.466479 \\ \text { H, } & 0.193906, & 0.469105, & 2.168161\end{array}$



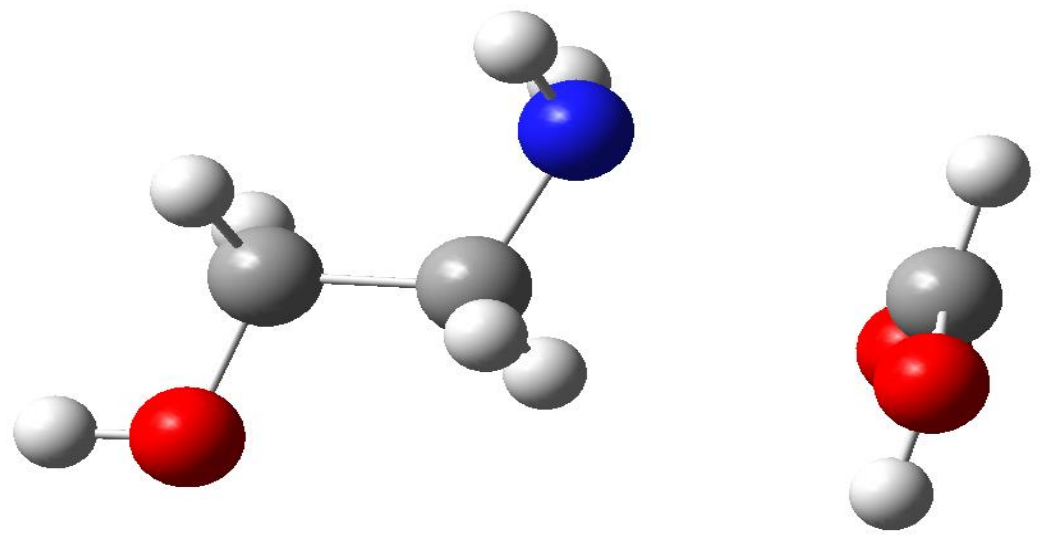

Formula:

Charge:

C3H9NO3

Energy:

0

Gibbs:

$-400.196538$

a.u

Multiplicity:

$-400.245144$

a.u

Dipole:

3.3970

Setup:

Formula:

Charge:

B3LYP, def2TZVP

C3H9NO3

0

Energy:

-400.209668 a.u

Gibbs:

-400.258634 a.u

Multiplicity: $\quad 1$

Dipole:

2.4047

Setup:

Formula:

M06, def2TZVP

C3H9NO3

Charge:

0

Energy:

-399.959726 a.u

Gibbs:

-400.007886 a.u

Multiplicity: $\quad 1$

Dipole:

3.3107

$\begin{array}{lrrr}\text { O, } & 2.191151, & 0.812719, & 0.317617 \\ \text { C, } & -0.410355, & 2.466641, & -1.288534 \\ \text { H, } & 0.681117, & 2.458451, & -1.260284 \\ \text { H, } & -0.712657, & 1.813599, & -2.109707 \\ \text { C, } & -0.883323, & 3.883983, & -1.582195 \\ \text { H, } & -0.576453, & 4.548182, & -0.765320 \\ \text { H, } & -1.978382, & 3.903246, & -1.635905 \\ \text { O, } & -0.301749, & 4.293451, & -2.822014 \\ \text { H, } & -0.591739, & 5.190041, & -3.015025 \\ \text { H, } & -1.924130, & 1.878672, & -0.036004 \\ \text { H, } & -0.634588, & 2.486045, & 0.747906 \\ \text { N, } & -0.910854, & 1.904421, & -0.035033 \\ \text { C, } & 1.329233, & -0.006090, & 0.124572 \\ \text { H, } & 0.650821, & -0.395535, & 0.886568 \\ \text { O, } & 1.081131, & -0.590060, & -1.054854 \\ \text { H, } & 1.689037, & -0.222143, & -1.716522\end{array}$




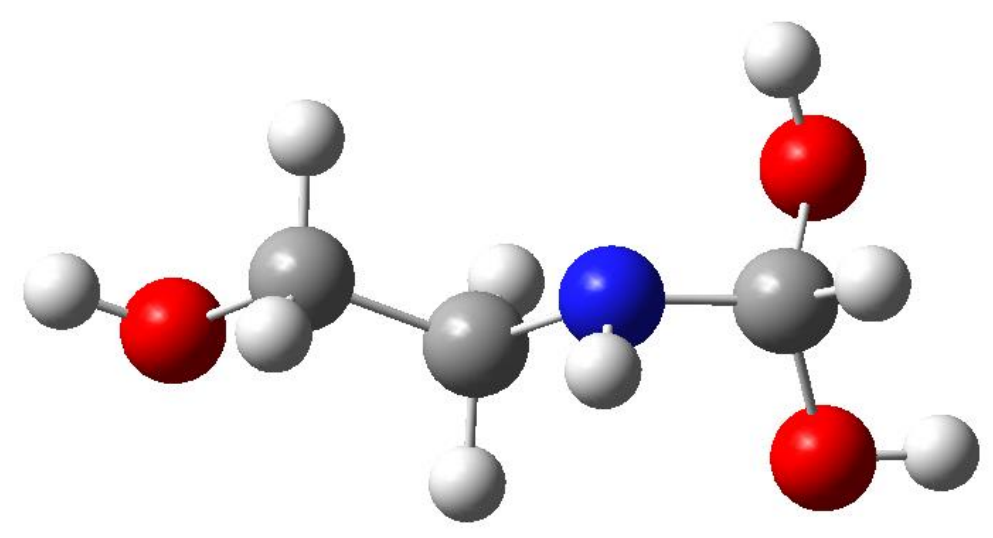

$\begin{array}{lll}\text { Formula: } & \text { C3H9NO3 } & \\ \text { Charge: } & 0 & \\ \text { Energy: } & -400.181923 & \text { a.u } \\ \text { Gibbs: } & -400.224701 & \text { a.u }\end{array}$

Multiplicity: $\quad 1$

Dipole: $\quad 3.6583$

Setup: $\quad$ B3LYP, def2TZVP

Formula: C3H9NO3

Charge: $\quad 0$

Energy: $\quad-400.195371$ a.u

Gibbs: $\quad-400.238139$ a.u

Multiplicity: $\quad 1$

Dipole: $\quad 3.8177$

Setup: M06, def2TZVP

Formula: $\quad \mathrm{C} 3 \mathrm{H} 9 \mathrm{NO} 3$

Charge: $\quad 0$

Energy: $\quad-399.947659$ a.u

Gibbs: $\quad-399.990193$ a.u

Multiplicity: $\quad 1$

Dipole: $\quad 3.6363$

$\begin{array}{lrrr}\text { O, } & 1.639235, & 1.341289, & 0.517801 \\ \text { C, } & -0.154360, & 2.525500, & -1.537453 \\ \text { H, } & 0.856412, & 2.894089, & -1.368842 \\ \text { H, } & -0.176118, & 2.070206, & -2.532775 \\ \text { C, } & -1.124550, & 3.692694, & -1.500570 \\ \text { H, } & -1.045732, & 4.202168, & -0.534749 \\ \text { H, } & -2.152712, & 3.325166, & -1.602458 \\ \text { O, } & -0.788010, & 4.566841, & -2.578952 \\ \text { H, } & -1.401949, & 5.307181, & -2.573562 \\ \text { H, } & -1.329579, & 1.070345, & -0.668846 \\ \text { H, } & 1.282231, & 1.832072, & 1.267413 \\ \text { N, } & -0.476885, & 1.577352, & -0.470279 \\ \text { C, } & 0.557245, & 0.665153, & -0.099646 \\ \text { H, } & 0.123797, & -0.066797, & 0.591896 \\ \text { O, } & 1.095635, & 0.008001, & -1.227165 \\ \text { H, } & 1.793599, & -0.585637, & -0.926549\end{array}$



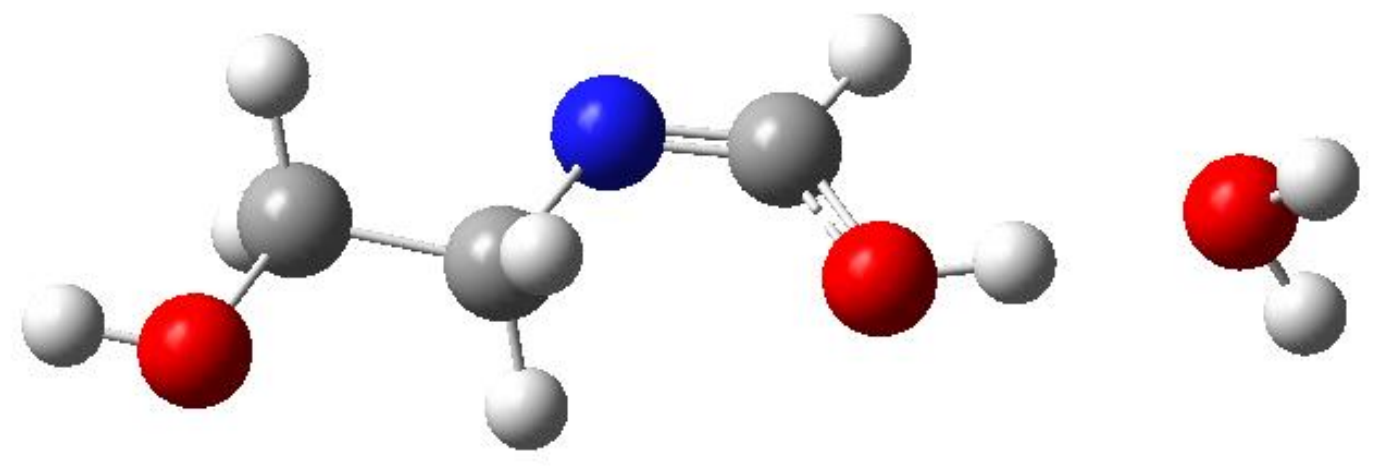

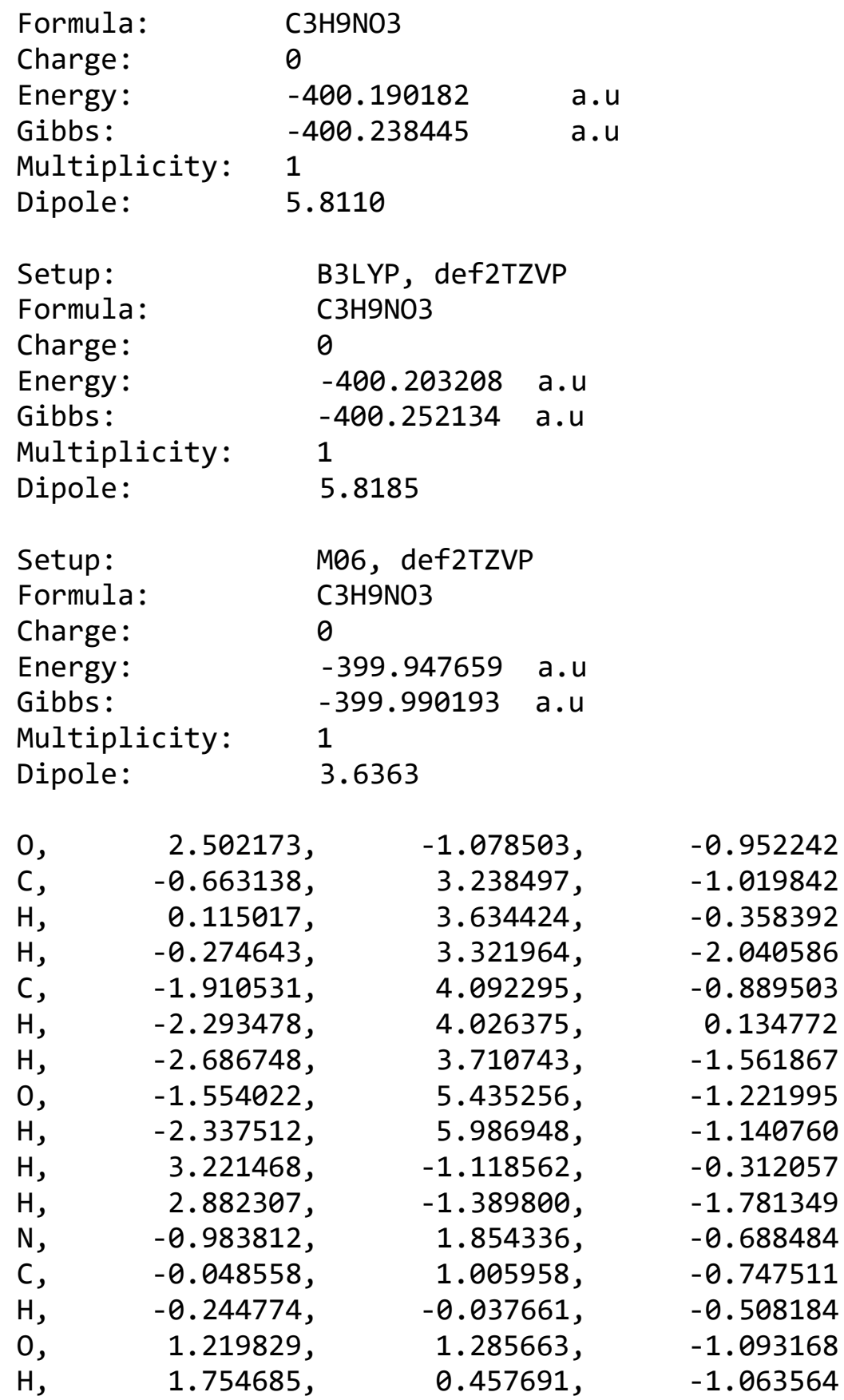




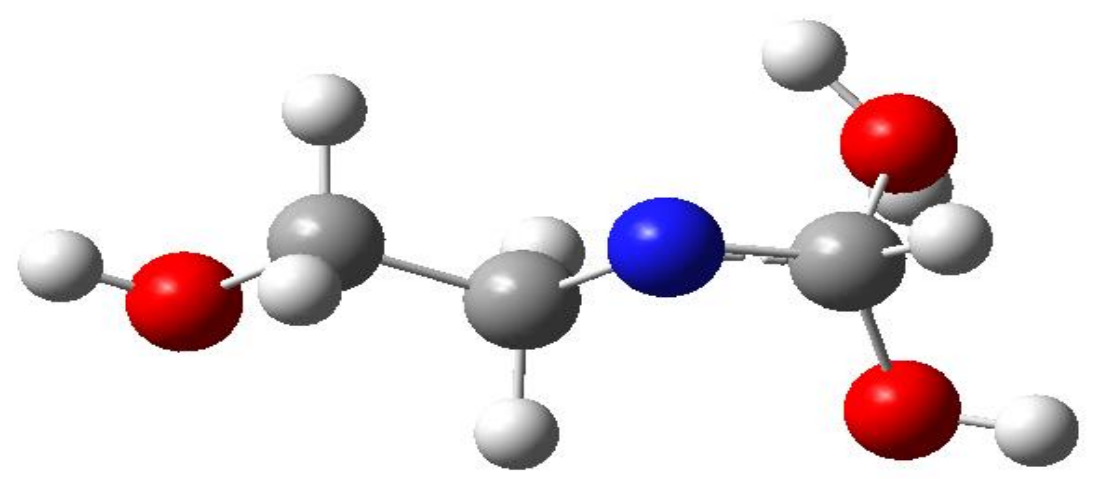

$\begin{array}{lll}\text { Formula: } & \text { C3H9N03 } & \\ \text { Charge: } & 0 & \\ \text { Energy: } & -400.078365 & \text { a.u } \\ \text { Gibbs: } & -400.117382 & \text { a.u }\end{array}$

Multiplicity: 1

Dipole: $\quad 7.3533$

Setup: $\quad$ B3LYP, def2TZVP

Formula: $\quad$ C3H9NO3

Charge: $\quad 0$

Energy: $\quad-400.091915$ a.u

Gibbs: $\quad-400.130905$ a.u

Multiplicity: $\quad 1$

Dipole: $\quad 7.4843$

Setup: $\quad$ M06, def2TZVP

Formula: C3H9NO3

Charge: $\quad 0$

Energy: $\quad-399.947659$ a.u

Gibbs: $\quad-399.990193$ a.u

Multiplicity: $\quad 1$

Dipole: $\quad 3.6363$

$\begin{array}{lrrr}\text { O, } & 1.325637, & 0.438540, & -0.429219 \\ \text { C, } & -0.312907, & 2.804761, & -1.110150 \\ \text { H, } & 0.660502, & 3.051314, & -0.648749 \\ \text { H, } & -0.244946, & 3.173245, & -2.145560 \\ \text { C, } & -1.365850, & 3.630197, & -0.393296 \\ \text { H, } & -1.406573, & 3.332260, & 0.660577 \\ \text { H, } & -2.346953, & 3.423864, & -0.835515 \\ \text { O, } & -1.028653, & 5.017389, & -0.520216 \\ \text { H, } & -1.701881, & 5.534520, & -0.068641 \\ \text { H, } & 1.003890, & 0.802077, & 0.418470 \\ \text { H, } & 2.153255, & 0.878130, & -0.703977 \\ \text { N, } & -0.681823, & 1.398543, & -1.037585 \\ \text { C, } & 0.277305, & 0.539805, & -1.396506 \\ \text { H, } & -0.087989, & -0.486439, & -1.478948 \\ \text { O, } & 1.074837, & 0.855163, & -2.578522 \\ \text { H, } & 1.380411, & 0.032255, & -2.976896\end{array}$




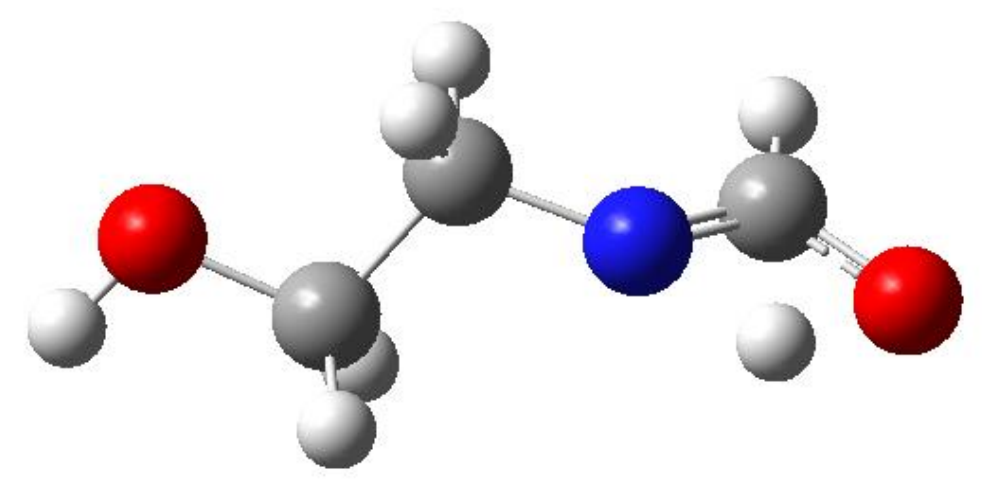

Formula:

Charge:

C3H7NO2

Energy:

0

Gibbs:

$-323.691103$

a.u

Multiplicity:

$-323.72969$

a.u

Dipole:

1

3.0760

Setup:

Formula:

Charge:

Energy:

Gibbs:

B3LYP, def2TZVP

C3H7NO2

0

-323.702026 a.u

Multiplicity: $\quad 1$

Dipole:

-323.740606 a.u

1

3.1357

$\begin{array}{lll}\text { Setup: } & \text { M06, def2TZVP } \\ \text { Formula: } & \text { C3H7NO2 } & \\ \text { Charge: } & 0 & \\ \text { Energy: } & -645.887813 & \text { a.u } \\ \text { Gibbs: } & -645.944367 & \text { a.u } \\ \text { Multiplicity: } & 1 & \\ \text { Dipole: } & 5.7101 & \end{array}$

$\begin{array}{lrrr}\text { O, } & 0.264657, & 0.380694, & 1.201841 \\ \text { C, } & 0.264235, & 2.806863, & -1.348198 \\ \text { H, } & 1.082271, & 3.528316, & -1.304734 \\ \text { H, } & 0.413579, & 2.188440, & -2.236276 \\ \text { C, } & -1.051392, & 3.564534, & -1.470825 \\ \text { H, } & -1.218891, & 4.153715, & -0.563026 \\ \text { H, } & -1.875965, & 2.850529, & -1.568747 \\ \text { O, } & -0.947679, & 4.400270, & -2.621403 \\ \text { H, } & -1.770153, & 4.889333, & -2.719398 \\ \text { H, } & 0.288866, & 1.698774, & 1.170883 \\ \text { N, } & 0.284737, & 1.988838, & -0.154197 \\ \text { C, } & 0.257275, & 0.701141, & -0.052348 \\ \text { H, } & 0.236510, & -0.030879, & -0.854766\end{array}$




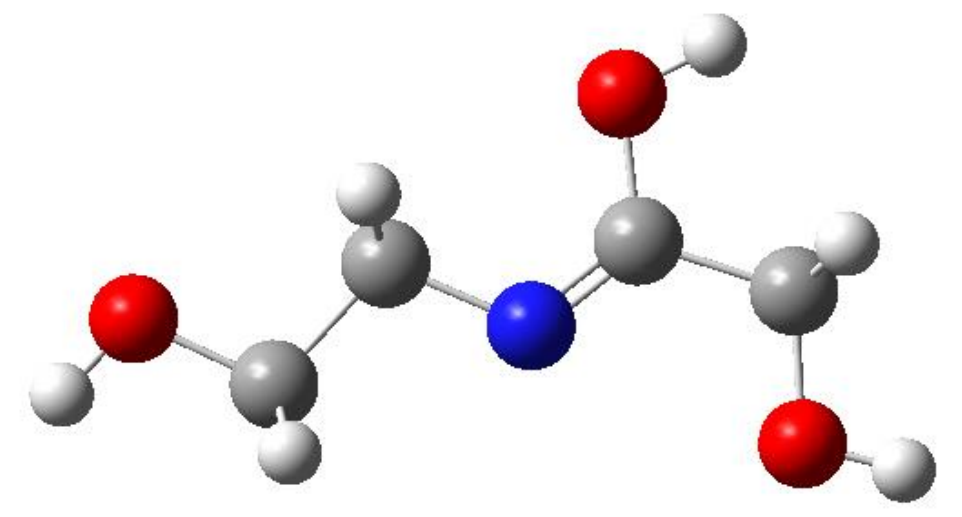

Formula:

C3H7NO2

Charge:

0

Energy:

$-323.73678$

a.u

Gibbs:

$-323.775684$

a.u

Multiplicity: 1

Dipole:

2.6952

Setup:

Formula:

Charge:

B3LYP, def2TZVP

Energy:

Gibbs:

$\mathrm{C} 3 \mathrm{H} 7 \mathrm{NO} 2$

0

-323.747609 a.u

Multiplicity: $\quad 1$

Dipole:

-323.786425 a.u

1

2.7464

Setup:

Formula:

M06, def2TZVP

Charge:

$\mathrm{C} 3 \mathrm{H} 7 \mathrm{NO} 2$

Energy:

Gibbs:

0

-323.538198 a.u

-323.576928 a.u

Multiplicity: 1

Dipole:

2.7238

$\begin{array}{lrrr}\text { C, } & -0.369808, & 2.801090, & -1.159342 \\ \text { H, } & 0.646018, & 3.050466, & -0.836129 \\ \text { H, } & -0.443485, & 3.092927, & -2.211940 \\ \text { C, } & -1.366589, & 3.604937, & -0.345160 \\ \text { H, } & -1.284962, & 3.326696, & 0.711142 \\ \text { H, } & -2.383807, & 3.369156, & -0.676154 \\ \text { O, } & -1.066458, & 4.987254, & -0.540602 \\ \text { H, } & -1.690664, & 5.510021, & -0.028790 \\ \text { N, } & -0.639676, & 1.376979, & -0.989513 \\ \text { C, } & 0.093137, & 0.553173, & -1.596534 \\ \text { H, } & -0.073561, & -0.516538, & -1.497494 \\ \text { O, } & 1.124261, & 0.912013, & -2.403417 \\ \text { H, } & 1.549933, & 0.125981, & -2.766021\end{array}$




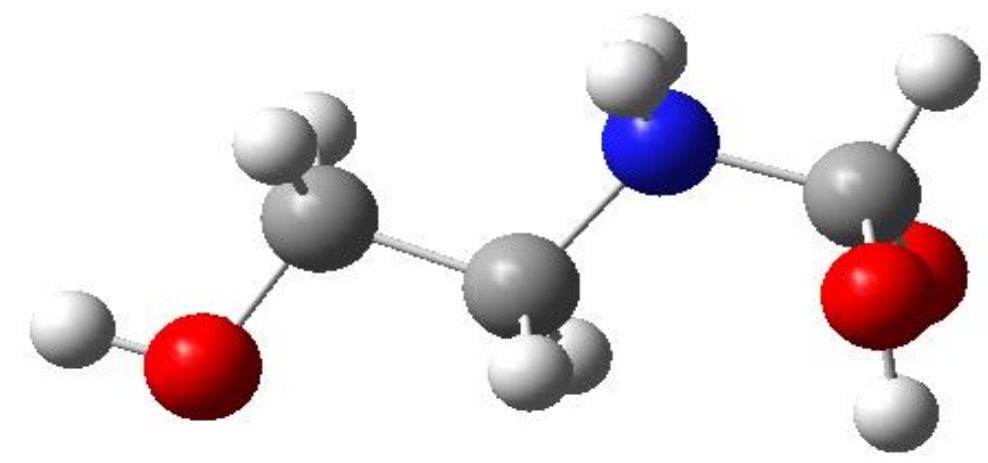

Formula:

C3H9NO3

Charge:

0

Energy:

-400.160781 a.u

Gibbs:

$-400.201076$

a.u

Multiplicity: $\quad 1$

Dipole:

9.4832

Setup:

Formula:

Charge:

B3LYP, def2TZVP

C3H9NO3

0

Energy:

-400.175576 a.u

-400.215979 a.u

Gibbs:

1

Dipole:

9.7200

Setup:

Formula:

M06, def2TZVP

Charge:

C3H9NO3

Energy:

0

Gibbs:

-399.924642 a.u -399.964981 a.u

Multiplicity: $\quad 1$

Dipole:

9.5491

$\begin{array}{lllr}\text { O, } & 1.864591, & 0.965352, & 0.175847 \\ \text { C, } & 0.049148, & 2.492274, & -1.400424 \\ \text { H, } & 1.096219, & 2.764651, & -1.319857 \\ \text { H, } & -0.089035, & 1.845018, & -2.260805 \\ \text { C, } & -0.835568, & 3.721457, & -1.498721 \\ \text { H, } & -0.702677, & 4.353208, & -0.613169 \\ \text { H, } & -1.890079, & 3.429915, & -1.551510 \\ \text { O, } & -0.427172, & 4.397643, & -2.681232 \\ \text { H, } & -0.978766, & 5.178047, & -2.793926 \\ \text { H, } & -1.232401, & 1.336050, & -0.251612 \\ \text { H, } & -0.244761, & 2.295076, & 0.635016 \\ \text { N, } & -0.275348, & 1.685184, & -0.182955 \\ \text { C, } & 0.647420, & 0.572418, & 0.083787 \\ \text { H, } & 0.194033, & 0.077920, & 0.972285 \\ \text { O, } & 0.364966, & -0.368697, & -1.004471 \\ \text { H, } & 1.157690, & -0.319895, & -1.552986\end{array}$



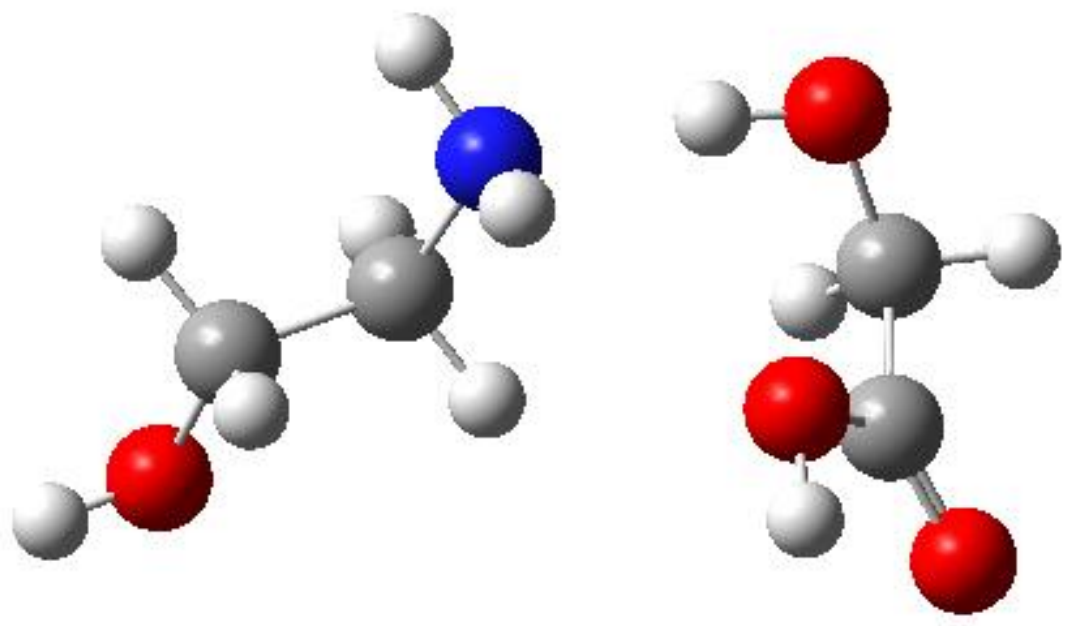

\begin{tabular}{lll} 
Formula: & \multicolumn{2}{l}{ C4H11NO3 } \\
Charge: & 0 & \\
Energy: & -439.502262 & a.u \\
Gibbs: & -439.555185 & a.u \\
Multiplicity: & 1 & \\
Dipole: & 3.8376 & \\
& & \\
Setup: & B3LYP, def2TZVP \\
Formula: & C4H11NO3 & \\
Charge: & 0 & \\
Energy: & -439.523561 & a.u \\
Gibbs: & -439.576776 & a.u \\
Multiplicity: & 1 & \\
Dipole: & 4.7221 & \\
& & \\
Setup: & M06, def2TZVP \\
Formula: & C4H11NO3 & \\
Charge: & 0 & \\
Energy: & -439.237743 & a.u \\
Gibbs: & -439.292853 & a.u \\
Multiplicity: & 1 & \\
Dipole: & 4.8579 &
\end{tabular}

$\begin{array}{lllr}\text { O, } & -3.438008, & 3.248231, & 0.257440 \\ \text { C, } & -0.132108, & 4.488996, & -1.021071 \\ \text { H, } & -0.634511, & 3.959180, & -0.210379 \\ \text { H, } & -0.589651, & 4.145567, & -1.949924 \\ \text { C, } & 1.341026, & 4.102801, & -1.024567 \\ \text { H, } & 1.803231, & 4.411129, & -0.077928 \\ \text { H, } & 1.856625, & 4.633635, & -1.835844 \\ \text { O, } & 1.419298, & 2.690259, & -1.202116 \\ \text { H, } & 2.341837, & 2.422341, & -1.172911 \\ \text { H, } & 0.060821, & 6.447121, & -1.614942 \\ \text { H, } & -0.016377, & 6.267553, & 0.002155\end{array}$




$\begin{array}{llll}\text { N, } & -0.389728, & 5.922234, & -0.874323 \\ \text { C, } & -3.504426, & 3.868310, & -0.775597 \\ \text { O, } & -3.254059, & 3.295369, & -1.978449 \\ \text { H, } & -3.006519, & 2.373550, & -1.810836 \\ \text { C, } & -3.812817, & 5.328339, & -0.904342 \\ \text { H, } & -2.856809, & 5.856952, & -0.965524 \\ \text { H, } & -4.381980, & 5.533823, & -1.808398 \\ \text { H, } & -4.350363, & 5.664533, & -0.022637\end{array}$




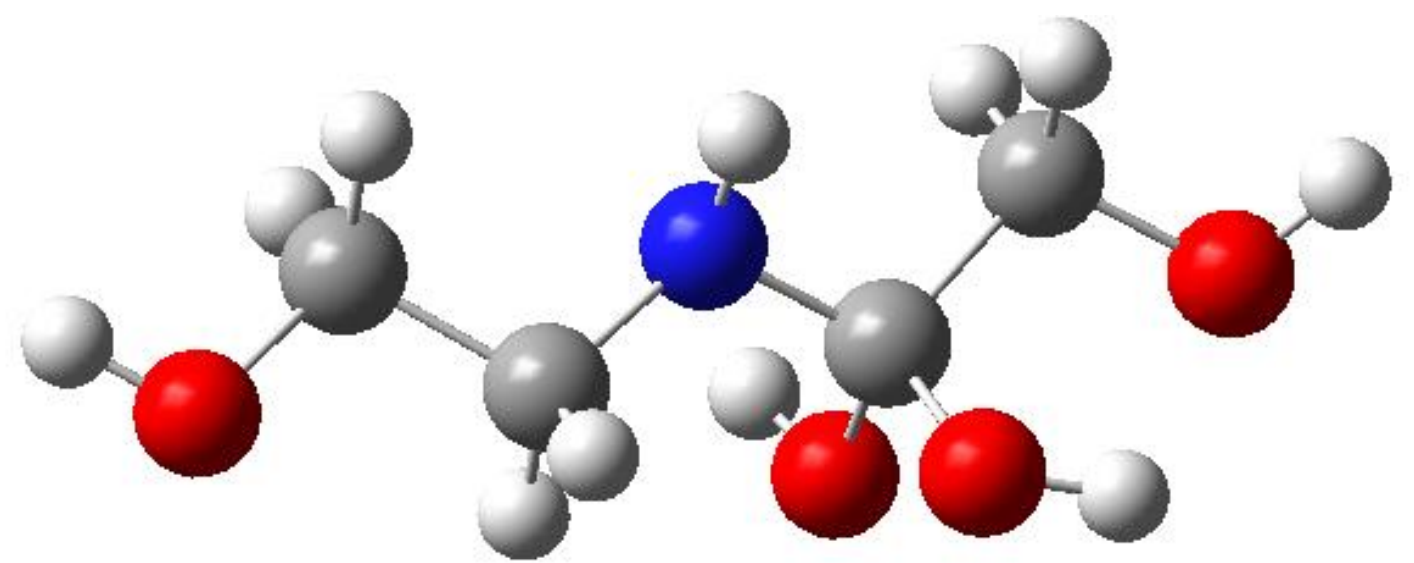

Formula:

Charge:

Energy:

Gibbs:

Multiplicity:

Dipole:

Setup:

Formula:

Charge:

Energy:

Gibbs:

Multiplicity:

Dipole:

Setup:

Formula:

Charge:

Energy:

Gibbs:

Multiplicity:

Dipole:
C4H11NO4

0

$-514.730044$

$-514.778225$

a.u

a.u

1

5.7779

B3LYP, def2TZVP

$\mathrm{C} 4 \mathrm{H} 11 \mathrm{NO} 4$

0

-514.747159 a.u

-514.795432 a.u

1

5.9553

M06, def2TZVP

$\mathrm{C} 4 \mathrm{H} 11 \mathrm{NO} 4$

0

-514.433916 a.u

-514.481543 a.u

1

5.9022

$\begin{array}{lrrr}\text { O, } & 1.718077, & 1.402407, & 0.481491 \\ \text { C, } & -0.110294, & 2.541435, & -1.468345 \\ \text { H, } & 0.860564, & 2.980625, & -1.246209 \\ \text { H, } & -0.041159, & 2.070685, & -2.454659 \\ \text { C, } & -1.157697, & 3.639590, & -1.510630 \\ \text { H, } & -1.177681, & 4.162351, & -0.548898 \\ \text { H, } & -2.150185, & 3.203083, & -1.675323 \\ \text { O, } & -0.808559, & 4.523300, & -2.576667 \\ \text { H, } & -1.468187, & 5.221819, & -2.621211 \\ \text { H, } & -1.268694, & 1.062104, & -0.652601 \\ \text { H, } & 1.387345, & 1.985487, & 1.175215 \\ \text { N, } & -0.437602, & 1.585986, & -0.407828 \\ \text { C, } & 0.608885, & 0.677425, & -0.036229 \\ \text { O, } & 1.098953, & -0.036148, & -1.146700\end{array}$




$\begin{array}{lrrr}\text { H, } & 1.576040, & -0.797981, & -0.787404 \\ \text { C, } & 0.071863, & -0.307739, & 1.014332 \\ \text { H, } & -0.846236, & -0.763341, & 0.638093 \\ \text { H, } & -0.155065, & 0.236375, & 1.934070 \\ \text { O, } & 1.077849, & -1.294662, & 1.237571 \\ \text { H, } & 0.651425, & -2.136772, & 1.416891\end{array}$




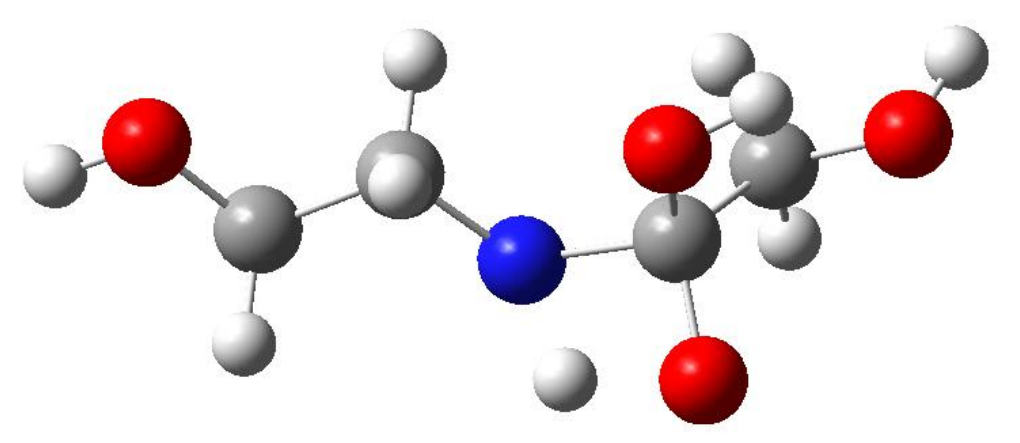

\begin{tabular}{|c|c|c|}
\hline Formula: & C4H11NO4 & \\
\hline Charge: & 0 & \\
\hline Energy: & -514.682674 & a.u \\
\hline Gibbs: & -514.730661 & a.u \\
\hline Multiplicity: & 1 & \\
\hline Dipole: & 7.7961 & \\
\hline Setup: & \multicolumn{2}{|c|}{ B3LYP, def2TZVP } \\
\hline Formula: & \multicolumn{2}{|l|}{ C4H11N04 } \\
\hline Charge: & \multicolumn{2}{|l|}{0} \\
\hline Energy: & -514.699640 & a.u \\
\hline Gibbs: & -514.748461 & a.u \\
\hline Multiplicity: & \multicolumn{2}{|l|}{1} \\
\hline Dipole: & \multicolumn{2}{|l|}{7.9755} \\
\hline Setup: & \multicolumn{2}{|l|}{ M06, def2TZVP } \\
\hline Formula: & \multicolumn{2}{|l|}{$\mathrm{C} 4 \mathrm{H} 11 \mathrm{NO} 4$} \\
\hline Charge: & \multicolumn{2}{|l|}{0} \\
\hline Energy: & -514.382883 & a.u \\
\hline Gibbs: & -514.431331 & $\mathrm{a} \cdot \mathrm{u}$ \\
\hline Multiplicity: & 1 & \\
\hline Dipole: & 8.0227 & \\
\hline
\end{tabular}

$\begin{array}{lrlr}\text { O, } & 0.749392, & 1.005197, & 1.471656 \\ \mathrm{C}, & -0.126068, & 2.516231, & -1.436908 \\ \mathrm{H}, & 0.885947, & 2.906679, & -1.382977 \\ \mathrm{H}, & -0.200424, & 1.883725, & -2.322190 \\ \mathrm{C}, & -1.119904, & 3.661363, & -1.526708 \\ \mathrm{H}, & -1.034243, & 4.294311, & -0.637482 \\ \mathrm{H}, & -2.143070, & 3.270041, & -1.565921 \\ \mathrm{O}, & -0.801451, & 4.383598, & -2.712190 \\ \mathrm{H}, & -1.405403, & 5.128748, & -2.786982 \\ \mathrm{H}, & -1.337178, & 1.385890, & -0.200998 \\ \mathrm{H}, & 0.015072, & 1.971303, & 0.927589 \\ \mathrm{~N}, & -0.376700, & 1.719944, & -0.225982 \\ \mathrm{C}, & 0.586905, & 0.589693, & 0.191172 \\ \mathrm{O}, & 1.734930, & 0.688256, & -0.626765\end{array}$




$\begin{array}{lrlr}\text { H, } & 2.126285, & -0.195826, & -0.637999 \\ \text { C, } & -0.050857, & -0.790552, & -0.001658 \\ \text { H, } & -0.881884, & -0.895840, & 0.700107 \\ \text { H, } & -0.422439, & -0.884246, & -1.025005 \\ \text { O, } & 0.972542, & -1.752221, & 0.256657 \\ \text { H, } & 0.737355, & -2.578912, & -0.174528\end{array}$




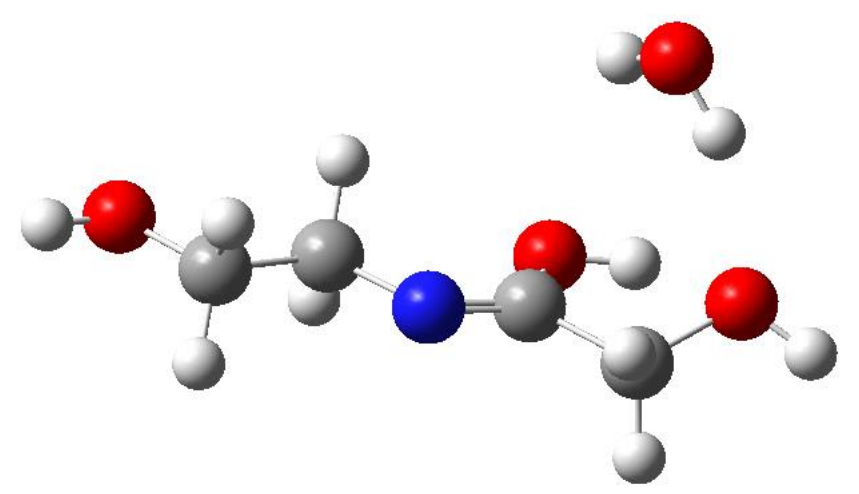

\begin{tabular}{|c|c|c|}
\hline Formula: & C4H11NO4 & \\
\hline Charge: & 0 & \\
\hline Energy: & -514.735444 & \\
\hline Gibbs: & -514.788748 & \\
\hline Multiplicity: & 1 & \\
\hline Dipole: & 5.5522 & \\
\hline Setup: & \multicolumn{2}{|c|}{ B3LYP, def2TZVP } \\
\hline Formula: & \multicolumn{2}{|l|}{ C4H11NO4 } \\
\hline Charge: & \multicolumn{2}{|l|}{0} \\
\hline Energy: & -514.752630 & a.u \\
\hline Gibbs: & -514.806901 & a.u \\
\hline Multiplicity: & \multicolumn{2}{|l|}{1} \\
\hline Dipole: & \multicolumn{2}{|l|}{5.6672} \\
\hline Setup: & \multicolumn{2}{|l|}{ M06, def2TZVP } \\
\hline Formula: & \multicolumn{2}{|l|}{$\mathrm{C} 4 \mathrm{H} 11 \mathrm{NO} 4$} \\
\hline Charge: & \multicolumn{2}{|l|}{0} \\
\hline Energy: & -514.433638 & a.u \\
\hline Gibbs: & -514.488646 & a.u \\
\hline Multiplicity: & 1 & \\
\hline Dipole: & 4.9940 & \\
\hline
\end{tabular}

$\begin{array}{lrrr}\text { O, } & 2.295312, & -0.176597, & 0.504050 \\ \mathrm{C}, & -0.534969, & 2.953603, & -1.208928 \\ \mathrm{H}, & 0.432502, & 3.173489, & -0.745456 \\ \mathrm{H}, & -0.447033, & 3.232186, & -2.264158 \\ \mathrm{C}, & -1.608085, & 3.803280, & -0.554065 \\ \mathrm{H}, & -1.688046, & 3.538261, & 0.505760 \\ \mathrm{H}, & -2.575466, & 3.597488, & -1.024921 \\ \mathrm{O}, & -1.233321, & 5.171276, & -0.718471 \\ \mathrm{H}, & -1.907558, & 5.722970, & -0.311186 \\ \mathrm{H}, & 2.443179, & 0.599341, & -0.046212 \\ \mathrm{H}, & 1.923699, & -0.826736, & -0.110379 \\ \mathrm{~N}, & -0.873136, & 1.544079, & -1.060991 \\ \mathrm{C}, & -0.096545, & 0.687516, & -1.560884 \\ \mathrm{O}, & 1.051462, & 1.006254, & -2.211421 \\ \mathrm{H}, & 1.489668, & 0.178558, & -2.467703\end{array}$




$\begin{array}{llll}\text { C, } & -0.416057, & -0.789255, & -1.503598 \\ \text { H, } & -1.052399, & -1.045724, & -2.354021 \\ \text { H, } & -0.954340, & -1.003002, & -0.581394 \\ \text { O, } & 0.822013, & -1.507351, & -1.574793 \\ \text { H, } & 0.660127, & -2.411803, & -1.859477\end{array}$




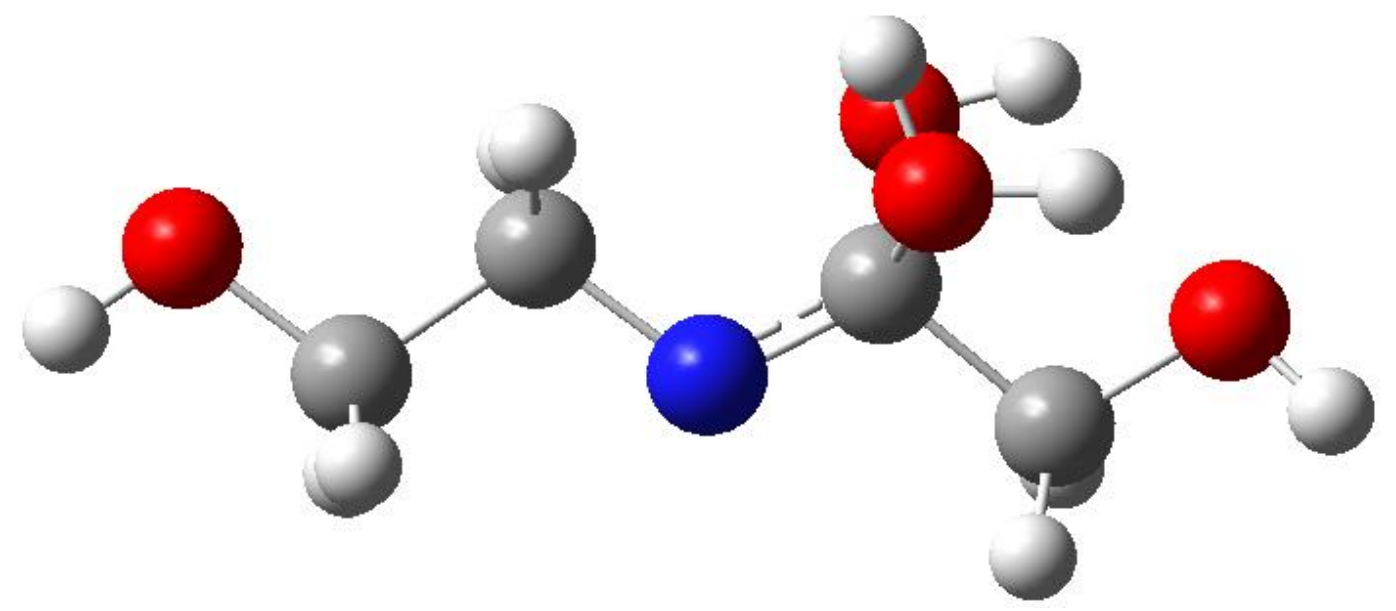

Formula:

Charge:

Energy:

Gibbs:

Multiplicity:

Dipole:

Setup:

Formula:

Charge:

Energy:

Gibbs:

Multiplicity:

Dipole:

Setup:

Formula:

Charge:

Energy:

Gibbs:

Multiplicity:

Dipole:
C4H11NO4

0

-514.628143 a.u

-514.675304 a.u

1

9.0242

B3LYP, def2TZVP

C4H11NO4

0

-514.645106 a.u

$-514.691866 a . u$

1

9.0919

M06, def2TZVP

$\mathrm{C} 4 \mathrm{H} 11 \mathrm{NO} 4$

0

-514.334149 a.u

-514.380137a.u

1

9.2238

$\begin{array}{lrrr}\text { O, } & 1.310081, & 0.439270, & -0.440440 \\ \text { C, } & -0.346465, & 2.852283, & -0.973955 \\ \text { H, } & 0.549469, & 3.085072, & -0.367388 \\ \text { H, } & -0.125349, & 3.234051, & -1.983551 \\ \text { C, } & -1.486045, & 3.681295, & -0.408631 \\ \text { H, } & -1.694926, & 3.360401, & 0.618150 \\ \text { H, } & -2.389006, & 3.502566, & -1.003448 \\ \text { O, } & -1.113756, & 5.064908, & -0.446998 \\ \text { H, } & -1.839325, & 5.582969, & -0.086985 \\ \text { H, } & 2.017041, & 1.090935, & -0.589806 \\ \text { H, } & 1.666146, & -0.487702, & -0.579541 \\ \text { N, } & -0.724521, & 1.453692, & -0.952985 \\ \text { C, } & 0.213241, & 0.594550, & -1.344725\end{array}$




$\begin{array}{llll}\text { O, } & 0.915949, & 0.947372, & -2.591614 \\ \text { H, } & 1.409782, & 0.181782, & -2.913271 \\ \text { C, } & -0.312653, & -0.847294, & -1.453016 \\ \text { H, } & -0.899836, & -0.936476, & -2.363544 \\ \text { H, } & -0.920782, & -1.074627, & -0.580636 \\ \text { O, } & 0.855255, & -1.699616, & -1.509819 \\ \text { H, } & 0.646705, & -2.577598, & -1.176043\end{array}$




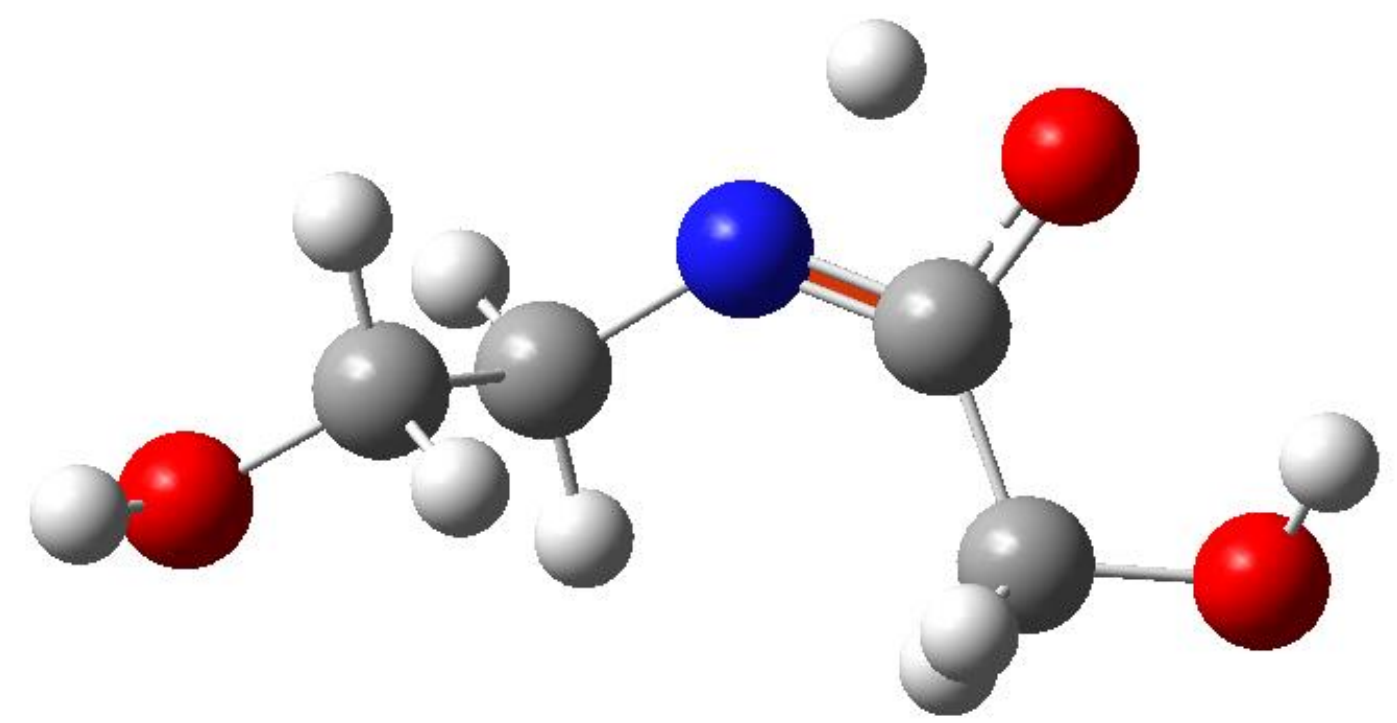

\begin{tabular}{|c|c|c|}
\hline Formula: & $\mathrm{C} 4 \mathrm{H} 9 \mathrm{NO} 3$ & \\
\hline Charge: & 0 & \\
\hline Energy: & -438.240392 & a. \\
\hline Gibbs: & -438.286357 & \\
\hline Multiplicity: & 1 & \\
\hline Dipole: & 3.7282 & \\
\hline Setup: & \multicolumn{2}{|c|}{ B3LYP, def2TZVP } \\
\hline Formula: & \multicolumn{2}{|c|}{ C4H9NO3 } \\
\hline Charge: & 0 & \\
\hline Energy: & -438.255307 & a.u \\
\hline Gibbs: & -438.300689 & a.u \\
\hline Multiplicity: & 1 & \\
\hline Dipole: & 3.8549 & \\
\hline Setup: & M06, def2TZVF & \\
\hline Formula: & C4H9NO3 & \\
\hline Charge: & 0 & \\
\hline Energy: & -438.021778 & a.u \\
\hline Gibbs: & -438.066256 & a.u \\
\hline Multiplicity: & 1 & \\
\hline Dipole: & 3.1542 & \\
\hline
\end{tabular}

$\begin{array}{lrlr}0, & 0.362858, & 0.314624, & 1.354679 \\ \text { C, } & 0.401728, & 2.753784, & -1.180768 \\ \text { H, } & 1.058363, & 3.605946, & -1.000797 \\ \text { H, } & 0.799558, & 2.210037, & -2.041346 \\ \text { C, } & -0.993042, & 3.270279, & -1.513938 \\ \text { H, } & -1.400342, & 3.802732, & -0.648242\end{array}$




$\begin{array}{lrrr}\text { H, } & -1.654223, & 2.425343, & -1.734089 \\ \text { O, } & -0.861320, & 4.133284, & -2.641238 \\ \text { H, } & -1.731316, & 4.475074, & -2.868389 \\ \text { H, } & 0.339212, & 1.614841, & 1.322496 \\ \text { N, } & 0.388774, & 1.917847, & 0.000311 \\ \text { C, } & 0.405405, & 0.623690, & 0.091466 \\ \text { C, } & 0.425259, & -0.428262, & -0.976665 \\ \text { H, } & 1.037857, & -0.093123, & -1.812710 \\ \text { H, } & -0.602130, & -0.534099, & -1.347406 \\ \text { O, } & 0.954617, & -1.658968, & -0.522637 \\ \text { H, } & 0.580519, & -1.838657, & 0.348263\end{array}$




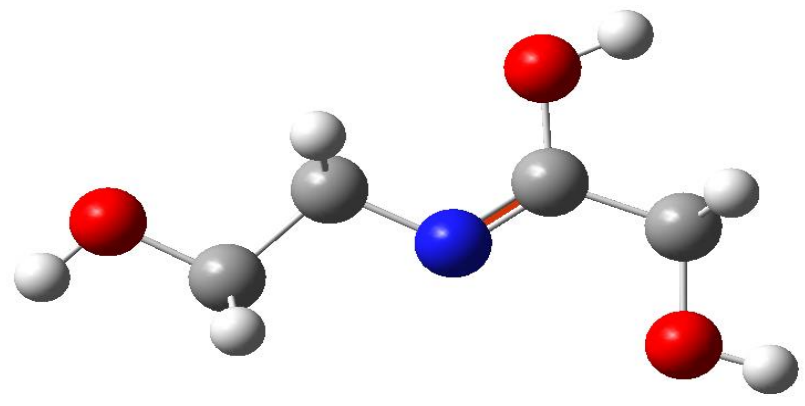

\begin{tabular}{lll} 
Formula: & \multicolumn{2}{l}{ C4H9NO3 } \\
Charge: & 0 & \\
Energy: & -438.281342 & a.u \\
Gibbs: & -438.327027 & a.u \\
Multiplicity: & 1 & \\
Dipole: & 4.8203 & \\
& & \\
Setup: & B3LYP, def2TZVP \\
Formula: & C4H9NO3 & \\
Charge: & 0 & \\
Energy: & -438.296282 & a.u \\
Gibbs: & -438.342041 & a.u \\
Multiplicity: & 1 & \\
Dipole: & 4.9434 & \\
& & \\
Setup: & M06, def2TZVP \\
Formula: & C4H9NO3 & \\
Charge: & 0 & \\
Energy: & -438.020574 & a.u \\
Gibbs: & -438.065716 & a.u \\
Multiplicity: & 1 & \\
Dipole: & 4.9370 &
\end{tabular}

$\begin{array}{lrrr}\text { C, } & -0.367340, & 2.779329, & -1.120577 \\ \text { H, } & 0.597602, & 2.916494, & -0.621674 \\ \text { H, } & -0.241105, & 3.136500, & -2.147865 \\ \text { C, } & -1.415565, & 3.625760, & -0.422477 \\ \text { H, } & -1.533948, & 3.282351, & 0.610934 \\ \text { H, } & -2.379647, & 3.504436, & -0.928143 \\ \text { O, } & -0.974570, & 4.983462, & -0.468876 \\ \text { H, } & -1.631111, & 5.532031, & -0.029708 \\ \text { N, } & -0.778886, & 1.381341, & -1.095903 \\ \text { C, } & -0.027043, & 0.526903, & -1.632786 \\ \text { O, } & 1.150010, & 0.871652, & -2.230447 \\ \text { H, } & 1.600974, & 0.097957, & -2.590608 \\ \text { C, } & -0.331643, & -0.949377, & -1.678208 \\ \text { H, } & -0.340200, & -1.254200, & -2.732888 \\ \text { H, } & 0.503495, & -1.475516, & -1.197532 \\ \text { O, } & -1.560827, & -1.230399, & -1.043273 \\ \text { H, } & -1.708775, & -2.179796, & -1.099339\end{array}$




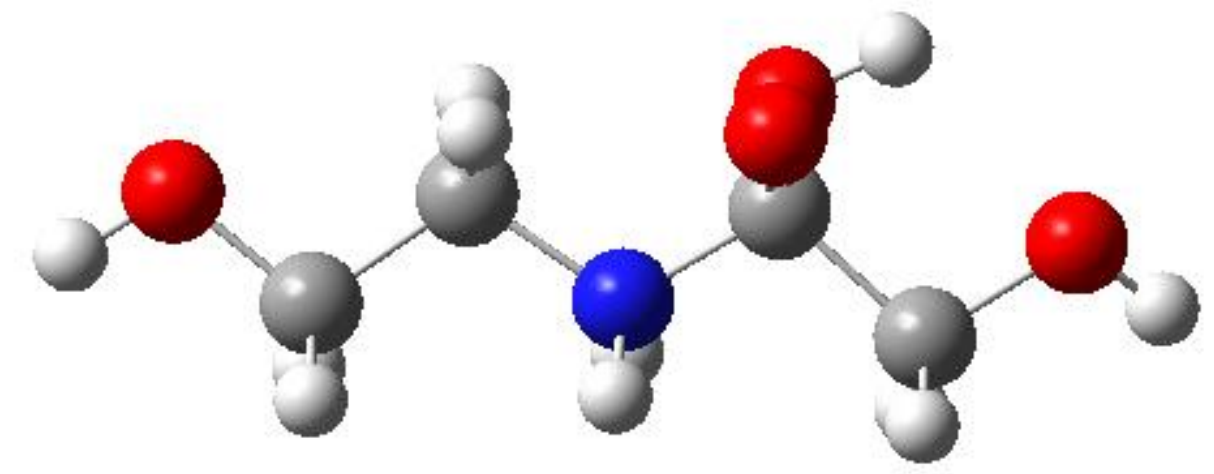

$\begin{array}{lll}\text { Formula: } & \text { C4H11NO4 } & \\ \text { Charge: } & 0 & \\ \text { Energy: } & -514.701609 & \text { a.u } \\ \text { Gibbs: } & -514.751038 & \text { a.u }\end{array}$

Multiplicity: 1

Dipole: $\quad 10.8483$

Setup: $\quad$ B3LYP, def2TZVP

Formula: $\quad$ C4H11NO4

Charge: $\quad 0$

Energy: $\quad-514.779184$ a.u

Gibbs: $\quad-514.831253$ a.u

Multiplicity: $\quad 1$

Dipole: $\quad 9.9520$

Setup: M06, def2TZVP

Formula: $\quad$ C4H11NO4

Charge: $\quad 0$

Energy: $\quad-514.460155$ a.u

Gibbs: $\quad-514.511530$ a.u

Multiplicity: $\quad 1$

Dipole: $\quad 9.8107$

$\begin{array}{lrrr}\text { O, } & 1.955693, & 1.107523, & 0.100458 \\ \text { C, } & 0.076463, & 2.558251, & -1.414805 \\ \text { H, } & 1.099829, & 2.895494, & -1.290240 \\ \text { H, } & 0.011278, & 1.932362, & -2.298374 \\ \text { C, } & -0.891043, & 3.724677, & -1.493755 \\ \text { H, } & -0.830348, & 4.327065, & -0.579896 \\ \text { H, } & -1.920176, & 3.362061, & -1.591651 \\ \text { O, } & -0.497414, & 4.476142, & -2.634676 \\ \text { H, } & -1.097699, & 5.221850, & -2.732266 \\ \text { H, } & -1.158625, & 1.284887, & -0.347018 \\ \text { H, } & -0.260324, & 2.281430, & 0.598303 \\ \text { N, } & -0.228891, & 1.690276, & -0.232998 \\ \text { C, } & 0.766786, & 0.638353, & 0.017972 \\ \text { O, } & 0.487854, & -0.239219, & -1.145440 \\ \text { H, } & 0.989179, & -1.040262, & -0.957764 \\ \text { C, } & 0.268800, & -0.144602, & 1.269970 \\ \text { H, } & -0.791827, & -0.401965, & 1.182930\end{array}$




$\begin{array}{llll}\text { H, } & 0.414106, & 0.485129, & 2.154590 \\ \mathrm{O}, & 1.065337, & -1.323792, & 1.347667 \\ \mathrm{H}, & 0.592292, & -1.975045, & 1.872938\end{array}$




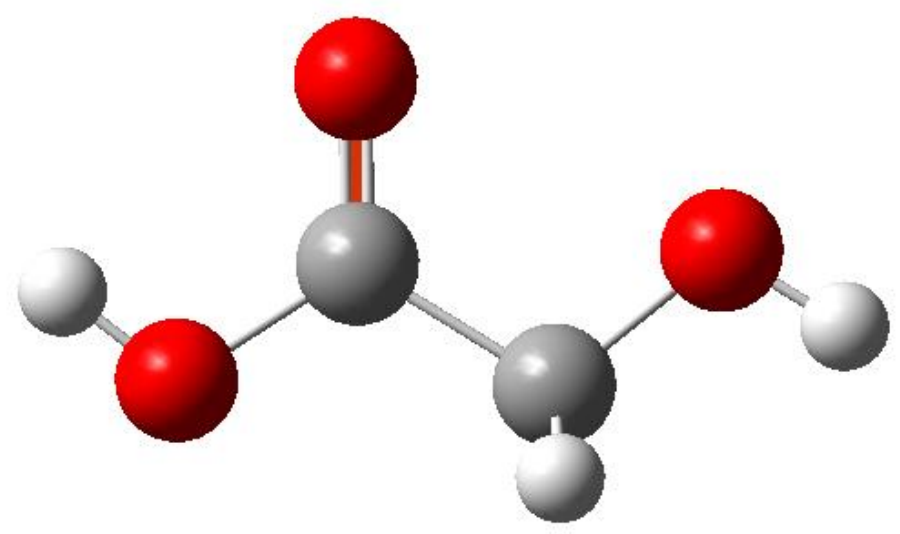

\begin{tabular}{|c|c|c|c|}
\hline Formula: & \multicolumn{3}{|l|}{$\mathrm{C} 2 \mathrm{H} 403$} \\
\hline Charge: & \multicolumn{3}{|l|}{0} \\
\hline Energy: & -304.359668 & \multicolumn{2}{|c|}{ a.u } \\
\hline Gibbs: & -304.394017 & \multicolumn{2}{|c|}{ a.u } \\
\hline Multiplicity: & \multicolumn{3}{|l|}{1} \\
\hline Dipole: & \multicolumn{3}{|l|}{3.9310} \\
\hline Setup: & \multicolumn{3}{|c|}{ B3LYP, def2TZVP } \\
\hline Formula: & \multicolumn{3}{|l|}{$\mathrm{C} 2 \mathrm{H} 403$} \\
\hline Charge: & \multicolumn{3}{|l|}{0} \\
\hline Energy: & \multicolumn{3}{|c|}{-304.372346 a.u } \\
\hline Gibbs: & \multicolumn{3}{|c|}{-304.406703 a.u } \\
\hline Multiplicity: & \multicolumn{3}{|c|}{1} \\
\hline Dipole: & \multicolumn{3}{|l|}{4.0677} \\
\hline Setup: & \multicolumn{3}{|c|}{ M06, def2TZVP } \\
\hline Formula: & \multicolumn{3}{|c|}{$\mathrm{C} 2 \mathrm{H} 4 \mathrm{O} 3$} \\
\hline Charge: & \multicolumn{3}{|l|}{0} \\
\hline Energy: & \multicolumn{3}{|c|}{-304.205004 a.u } \\
\hline Gibbs: & \multicolumn{3}{|c|}{-304.239167 a.u } \\
\hline Multiplicity: & \multicolumn{3}{|l|}{1} \\
\hline Dipole: & \multicolumn{3}{|l|}{3.9579} \\
\hline \multicolumn{2}{|c|}{0.664987} & 0.587292 & -0.127808 \\
\hline \multicolumn{2}{|c|}{ 1.867545, } & 0.652795 & -0.128074 \\
\hline \multicolumn{2}{|c|}{-0.142506} & 1.664915, & -0.127790 \\
\hline \multicolumn{2}{|c|}{0.411148} & 2.460698 & -0.127893 \\
\hline-0.16 & -0.67 & -0.678680 & -0.127849 \\
\hline-0.80 & -0.64 & 496, & 0.753824 \\
\hline-0.80 & -0.64 & 283 & -1.009323 \\
\hline 0.70 & -1.79 & 575, & -0.128110 \\
\hline 0.15 & -2.58 & 171 & -0.128124 \\
\hline
\end{tabular}




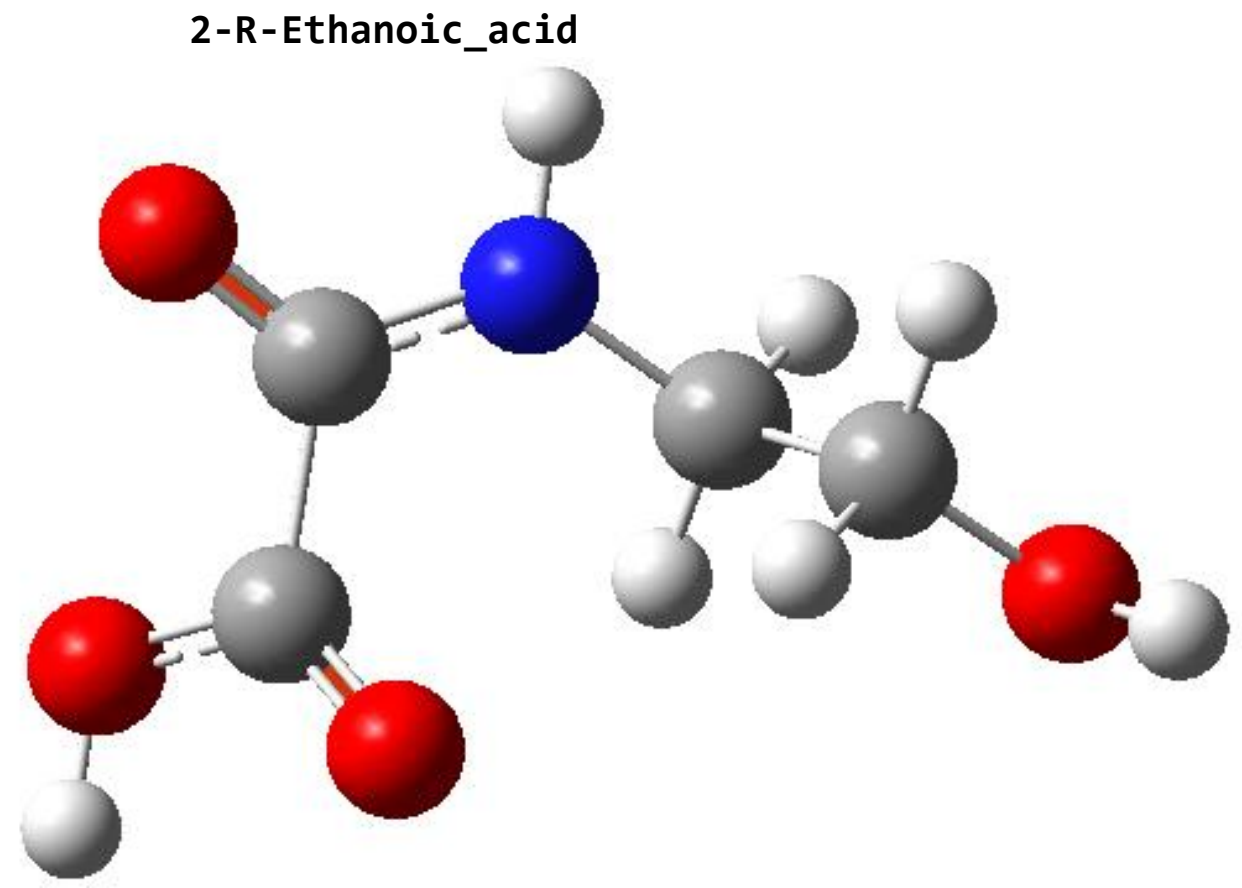

Formula:

Charge:

Energy:

Gibbs:

Multiplicity:

Dipole:

Setup:

Formula:

Charge:

Energy:

Gibbs:

Multiplicity:

Dipole:

Setup:

Formula:

Charge:

Energy:

Gibbs:

Multiplicity:

Dipole:
C4H7NO4

0

-512.390657 a.u

-512.436906 a.u

1

3.3806

B3LYP, def2TZVP

C4H7NO4

0

-512.409514 a.u

-512.456856 a.u

1

3.6941

M06, def2TZVP

$\mathrm{C} 4 \mathrm{H} 7 \mathrm{NO} 4$

0

-512.110130 a.u

-512.157003 a.u

1

3.5530

$\begin{array}{rrrr}\text { C, } & 0.789825, & 0.157424, & 0.180911 \\ \text { O, } & 1.872025, & -0.020326, & 0.682093 \\ \text { O, } & 0.085404, & 1.286706, & 0.263196 \\ \text { H, } & 0.589052, & 1.929262, & 0.789395 \\ \text { C, } & 0.090291, & -0.869548, & -0.723704 \\ \text { O, } & -0.217339, & -0.535955, & -1.860347 \\ \text { N, } & -0.090083, & -2.097518, & -0.214234 \\ \text { H, } & -0.490782, & -2.761335, & -0.861788 \\ \text { C, } & 0.240094, & -2.578694, & 1.120911\end{array}$




$\begin{array}{llll}\text { H, } & -0.558325, & -3.242746, & 1.450330 \\ \text { H, } & 0.276339, & -1.744039, & 1.816776 \\ \text { C, } & 1.568532, & -3.322128, & 1.145514 \\ \text { H, } & 1.536896, & -4.154803, & 0.434508 \\ \text { H, } & 2.366052, & -2.638463, & 0.843163 \\ \text { O, } & 1.751194, & -3.786053, & 2.480664 \\ \text { H, } & 2.591766, & -4.251059, & 2.530958\end{array}$




\section{Alternative basis set and functional data}

The following tables contain data for two additional computational setups (B3LYP, def2-TZVP and M06, def2-TZVP) for all of the figures in the main text.

\begin{tabular}{|l|l|l|l|l|l|l|l|}
\hline Fig. 3 & & & & & & & \\
\hline B3LYP, cc-pVTZ & 0 & 22.9 & -8.9 & 0 & -14.3 & -13.9 & -27.2 \\
\hline B3LYP, def2-tzvp & 0 & 23.6 & -8.25 & 0 & -12.8 & - & -29.4 \\
\hline M06, def2-tzvp & 0 & 22.5 & -10.2 & 0 & -13.2 & -12.1 & -27.2 \\
\hline
\end{tabular}

\begin{tabular}{|l|c|c|c|}
\hline Fig. 4 & & & \\
\hline B3LYP, cc-pVTZ & 0 & 39.5 & 6.3 \\
\hline B3LYP, def2-tzvp & 0 & 42.6 & 10.0 \\
\hline M06, def2-tzvp & 0 & 44.0 & 6.4 \\
\hline
\end{tabular}

\begin{tabular}{|l|l|l|l|l|}
\hline Fig 5. & & & & \\
\hline B3LYP, cc-pVTZ & 0 & -11.8 & 23.0 & 4.5 \\
\hline B3LYP, def2-tzvp & 0 & -10.4 & 24.8 & 6.1 \\
\hline M06, def2-tzvp & 0 & -10.3 & 27.1 & 6.1 \\
\hline
\end{tabular}

\begin{tabular}{|l|l|l|l|l|}
\hline Fig 6. & & & & \\
\hline B3LYP, cc-pVTZ & 0 & -7.1 & 21.7 & -23.6 \\
\hline B3LYP, def2-tzvp & 0 & -6.0 & 22.3 & -23.0 \\
\hline M06, def2-tzvp & 0 & -5.9 & 24.5 & -21.4 \\
\hline
\end{tabular}

\begin{tabular}{|l|l|l|l|l|}
\hline Fig 7 & & & & \\
\hline B3LYP, cc-pVTZ & 0 & -17.6 & -14.7 & -35.3 \\
\hline B3LYP, def2-tzvp & 0 & -15.8 & -12.6 & -33.3 \\
\hline M06, def2-tzvp & 0 & -14.1 & - & -31.0 \\
\hline
\end{tabular}

\begin{tabular}{|l|l|l|l|l|l|l|}
\hline Fig 8. & & & & & & \\
\hline B3LYP, cc-pVTZ & 0 & 27.3 & 7.9 & 9.2 & -2.8 & 0.2 \\
\hline B3LYP, def2-tzvp & 0 & 26.7 & 6.2 & 5.8 & -2.5 & -0.3 \\
\hline M06, def2-tzvp & 0 & 31.2 & 9.4 & 8.4 & -5.1 & -3.5 \\
\hline
\end{tabular}

\begin{tabular}{|l|l|l|l|l|l|l|}
\hline Fig. 9 & & & & & & \\
\hline B3LYP, cc-pVTZ & 0 & -3.7 & 30.2 & 2.7 & 32.8 & -6.7 \\
\hline B3LYP, def2-tzvp & 0 & -3.1 & 30.8 & -0.2 & 33.0 & -10.9 \\
\hline M06, def2-tzvp & 0 & -3.2 & 33.0 & 2.1 & 40.4 & -4.1 \\
\hline
\end{tabular}

\begin{tabular}{|l|l|l|l|l|l|l|l|l|l|}
\hline Fig 11. & & & & & & & & & \\
\hline B3LYP, cc-pVTZ & 0 & -3.7 & 22.9 & -7.5 & -3.5 & 26.2 & -5.2 & 25.5 & -10.9 \\
\hline $\begin{array}{l}\text { B3LYP, def2- } \\
\text { tzvp }\end{array}$ & 0 & -2.6 & 22.9 & -6.0 & -2.8 & 26.9 & -4.3 & 26.1 & -13.9 \\
\hline M06, def2-tzvp & 0 & -3.8 & 27.6 & -8.3 & - & 25.8 & -5.2 & 29.7 & -13.8 \\
\hline
\end{tabular}

\begin{tabular}{|l|l|l|l|l|l|l|l|l|l|l|}
\hline Fig 12. & & & & & & & & & & \\
\hline B3LYP, cc-pVTZ & 0 & -2.7 & 8.1 & 7.8 & 6.9 & 5.5 & -4.9 & -8.3 & -13.4 & -0.5 \\
\hline $\begin{array}{l}\text { B3LYP, def2- } \\
\text { tzvp }\end{array}$ & 0 & -2.2 & 8.2 & 8.1 & 7.3 & 5.5 & -3.9 & -7.6 & -12.0 & -0.2 \\
\hline M06, def2-tzvp & 0 & -2.61 & 10.2 & 10.1 & 8.4 & 9.6 & -4.0 & -8.2 & -12.0 & 0.3 \\
\hline
\end{tabular}




\begin{tabular}{|l|l|l|l|l|l|l|l|}
\hline Fig 13 & & & & & & & \\
\hline B3LYP, cc-pVTZ & 0 & -13.4 & -41.3 & -125.0 & -134.6 & -86.2 & -121.3 \\
\hline B3LYP, def2-tzvp & 0 & -12.0 & -41.4 & -123.8 & -131.2 & -75.1 & -118.3 \\
\hline M06, def2-tzvp & 0 & -12.1 & -43.6 & -128.0 & -133.8 & -71.4 & -117.9 \\
\hline
\end{tabular}

\begin{tabular}{|l|l|l|l|l|l|l|l|l|l|}
\hline Fig 16 & & & & & & & & & \\
\hline B3LYP, cc-pVTZ & 0 & -2.5 & 19.9 & 33.1 & 6.6 & 1.5 & 8.9 & 37.6 & -7.8 \\
\hline $\begin{array}{l}\text { B3LYP, def2- } \\
\text { tzvp }\end{array}$ & 0 & -1.8 & 19.6 & 33.5 & 7.2 & 2.3 & 8.6 & 37.2 & -8.7 \\
\hline M06, def2-tzvp & 0 & -1.7 & 20.3 & 33.9 & 5.9 & 3.3 & 9.1 & 37.5 & -7.6 \\
\hline
\end{tabular}

\begin{tabular}{|l|l|l|l|l|l|l|}
\hline Fig 17 & & & & & & \\
\hline B3LYP, cc-pVTZ & 0 & 50.2 & -3.7 & 0 & 67.8 & -3.4 \\
\hline B3LYP, def2-tzvp & 0 & 49.1 & -4.3 & 0 & 65.6 & -4.5 \\
\hline M06, def2-tzvp & 0 & 54.2 & -5.7 & 0 & 70.1 & -5.6 \\
\hline
\end{tabular}

\section{Alternative Solvent Model}

The following table contains data comparison data to that in the main text carried out using the SMD solvent model as opposed to PCM.

\begin{tabular}{|c|c|c|c|c|}
\hline & \multicolumn{4}{|c|}{ Activation energies / $\mathrm{kcalmol}^{-1}$} \\
\hline Reaction & $\begin{array}{l}\text { B3LYP, cc-pVTZ } \\
\text { PCM solvent } \\
\text { model }\end{array}$ & $\begin{array}{l}\text { B3LYP, def2-tzvp } \\
\text { PCM solvent } \\
\text { model }\end{array}$ & $\begin{array}{l}\text { M06, def2-tzvp } \\
\text { PCM solvent } \\
\text { model }\end{array}$ & $\begin{array}{l}\text { M06, def2-tzvp } \\
\text { SMD Solvent } \\
\text { model }\end{array}$ \\
\hline $\begin{array}{l}\text { Carbamate } \\
\text { formation (Fig3) }\end{array}$ & 0.4 & & 1.1 & 1.3 \\
\hline $\begin{array}{l}\text { Carbamic acid } \\
\text { formation (Fig 3) }\end{array}$ & 22.9 & 23.6 & 22.5 & 26.7 \\
\hline $\begin{array}{l}\text { OZD formation } \\
\text { from Ion pair (Fig } \\
\text { 4) }\end{array}$ & 39.5 & 42.6 & 44.0 & 42.4 \\
\hline $\begin{array}{l}\text { Isocyanate from } \\
\text { carbamic acid (Fig } \\
\text { 5) }\end{array}$ & 34.8 & 35.2 & 37.4 & 37.3 \\
\hline $\begin{array}{ll}\text { BHEU } & \text { formation } \\
\text { (Fig 6) } & \end{array}$ & 28.8 & 28.3 & 30.4 & 28.7 \\
\hline $\begin{array}{l}\text { HEEDA formation } \\
\text { (Fig 8) }\end{array}$ & 27.3 & 26.7 & 31.2 & 29.7 \\
\hline
\end{tabular}




\begin{tabular}{|l|c|c|c|c|}
\hline HEIA formation & 33.9 & 33.9 & 36.2 & 30.3 \\
(Fig 9) & 30.1 & 33.0 & 38.3 & 36.4 \\
\hline AHEIA formation & 26.6 & 25.5 & 31.4 & 29.0 \\
(Fig 11) & 29.7 & 29.7 & 37.0 & \\
& 30.7 & 30.4 & 34.9 & 34.9 \\
\hline Radical formation & 8.1 & 8.2 & 10.2 & 12.5 \\
(Fig 12) & 7.8 & 8.1 & 10.1 & 12.1 \\
& 6.9 & 7.3 & 8.4 & 11.5 \\
& 5.5 & 5.5 & 9.6 & 10.3 \\
\hline HEF formation (fig & 35.6 & 35.3 & 35.6 & 35.7 \\
16) & 36.1 & 34.9 & 34.2 & 30.3 \\
\hline HEPO formation & 50.2 & 49.1 & 54.2 & 64.5 \\
\hline (Fig 17) & 67.8 & 65.6 & 70.1 & \\
\hline
\end{tabular}

